will be published in Astrophysical Bulletin, 2015, vol. 70, No.1

\title{
Spectral atlas of A-type supergiants
}

\author{
V.G. Klochkova*, E.G. Sendzikas, E.L. Chentsov \\ Special Astrophysical Observatory RAS, Nizhnij Arkhyz, 369167 Russia \\ January 23, 2018

\begin{abstract}
Based on high-spectral-resolution observations $(R=60000)$ performed with the 6 -m BTA telescope in combination with the echelle spectrograph NES, we have studied the optical spectra of three A-type supergiants: a peculiar supergiant 3 Pup, a post-AGB star BD $+48^{\circ} 1220$, and a massive $\alpha \mathrm{Cyg}$, which belong to essentially different stages of evolution. A spectral atlas for these stars is prepared in the wavelength interval of 3920 to $6720 \AA$.
\end{abstract}

Key words. supergiants - stars: post-AGB - atlases - stars: individual: $3 \mathrm{Pup}, \mathrm{BD}+48^{\circ} 1220, \alpha \mathrm{Cyg}$

\section{Introduction}

The present paper is the next step in the study of evolved high luminosity stars (HLSs) at short final phases when a star is still powered by nuclear fusion. Our objects of study are both the most massive stars with initial masses greater than $10 M_{\odot}$ presumably at the stages of LBV, B[e] supergiants, yellow hypergiants (YHGs), the luminosity of which is often close to the Eddington limit, and the medium tmass stars with initial masses of 3-8 $M_{\odot}$ at the asymptotic giant branch (AGB) phase and the further $\checkmark$ post-AGB phase. The identification of stars of these types (LBV, B[e], YHG, AGB, or post-AGB), reliable determination of the fundamental parameters, and defining the evolutionary stage of HLSs Ohave become particularly important recently owing to the search for stars of these types in the Local Group galaxies (see [1, 2] and references therein).

The high luminosity stars of the types mentioned above, which fundamentally differ in mass and evolutionary stage, have similar observable properties: the characteristics of the optical and radio spec-

tra, large IR excesses, complex and variable velocity fields; this shows the instability of their extended atmospheres and expanding gaseous-dusty envelope. This overlapping of the observed characteristics of two types of objects represents the so-called "spectroscopic mimicry" problem.

The main feature of the optical spectra of the high and low mass HLSs are the absorption and emission profiles of the spectral lines, sometimes of an anomalous form, primarily of $\mathrm{H} \alpha$ lines. This can be a direct or an inverse $\mathrm{P}$ Cyg profile, an absorption with asymmetric emission wings, or a combination of different elements. We remind that the emission in $\mathrm{H} \alpha$ along with the IR excess is a principal selection criterion for post-AGB star candidates [3. The emission profile of $\mathrm{H} \alpha$ is a sign of the outflow of matter and/or pulsations. The differences in the profiles reflect the differences in the dynamic processes which occur in the extended atmospheres of some stars: the spherically symmetric outflow, infall of matter onto the photosphere, pulsations.

The best known example of an undefined evolutionary stage is the case of a peculiar supergiant V1302 Aql (IR-source IRC +10420), which had been considered a post-AGB stage representative for several decades until now. However, some authors referred this unique object to the most massive stars of the Galaxy, because some 30 years ago it was noted to be similar to $\eta$ Car, the distinguished LBV of the Galaxy. The cumulative results obtained with long-term observations using various techniques at the world's largest telescopes, and the 6-m BTA telescope among them [4, 5], revealed the actual

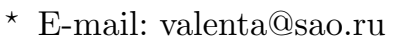


state of things. Now this object is conclusively classified as a massive star of extreme luminosity - a yellow hypergiant.

The poorly studied variable A supergiant V2324 Cyg identified with the IRAS 20572+4919 IR source can serve also as an example of an object with an undetermined status. The observable excess of radiation at about $12-60 \mu \mathrm{m}$ and the position on the IR-color diagram allow us to refer this object to the post-AGB stage, i.e., to consider it a protoplanetary nebula (PPN) with a dust shell. Using the BTA spectra for V2324 Cyg, with the method of model atmospheres we determined $T_{\text {eff }}=7500 \mathrm{~K}$, surface gravity $\log g=2.0$, microturbulence velocity $V_{t}=6.0 \mathrm{~km} \mathrm{~s}^{-1}$, and solar metallicity [6]. An unexpected feature for a PPN is lithium and sodium overabundance. The overabundance of these elements in the atmosphere allows us to classify V2324 Cyg as a star with the initial mass greater than $4 M_{\odot}$. Nuclear fusion of light metals ( $\mathrm{Li}, \mathrm{Na}, \mathrm{Al}$ ) is possible owing to the hot bottom burning (HBB) in the hot layers of the convective envelope in massive AGB-stars. The description of HBB and the necessary references are available in Ventura et al. [7.

Despite the large amount of obtained data, the evolutionary status of V2324 Cyg is still unclear. In the IR-color diagram, the IRAS 20572+4919 source is located in region IV, which is occupied by planetary and protoplanetary nebulae. In accordance with the chronological sequences presented by Lewis [8], the absence of maser radiation in $\mathrm{OH}$ and $\mathrm{H}_{2} \mathrm{O}$ lines indicates the approach of the object to the planetary nebula phase. This fact agrees with the conclusions from the publications in which a number of authors [9, 10] regard V2324 Cyg as a post-AGB star. However, some characteristics of the star do not correspond to the post-AGB stage; the first one is its low luminosity: the spectral classification indicates that its luminosity class is III. An excessive surface gravity, $\log g=2.06$, indicates the same. The $\mathrm{H} \alpha$ line profile and the high wind velocity, typical for supergiants, do not correspond to the post-AGB star status as well. As follows from Fig. 1 in Klochkova and Chentsov [6], the $\mathrm{H} \alpha$ profile with a strong variable emission more likely implies a high luminosity star with fast wind.

To gain experience and set up the classification criteria for HLSs, it would be useful to compare in detail their high resolution spectra in a wide wavelength interval. The present paper contains the atlas of the optical spectra of three supergiants similar in spectral type but different in luminosity: a massive supergiant $\alpha \mathrm{Cyg}$, a peculiar supergiant $3 \mathrm{Pup}$ (A2.7 Ib), and a protoplanetary nebula $\mathrm{BD}+48^{\circ} 1220$ (A4 Ia). The star 3 Pup (HD $62623=$ HR $2996=$ MWC $570=$ HIP 37677 ) is listed as an A2 Iabe supergiant in the Bright Star Catalogue [1].

After reviewing the main characteristics and parameters of the stars under study in Section 2, we will briefly present the used observational data (Section 3) and the compiled spectral atlas (Section 44).

\section{Characteristics of the studied A-type supergiants}

The studied A supergiants are presented in Table 1 in the same order as their spectra are presented in the atlas. Along with the star's name, the table gives the numbers of the associated IR-sources, the spectral type according to previous investigations of the stars, their evolutionary status, absolute magnitude $M_{V}$, visible magnitude $V$, and galactic coordinates $l, b$. The last two columns of this table show the mid-exposure time (in JD) and the heliocentric radial velocity $V_{r}$ measured by numerous symmetric absorptions in the spectra of the studied stars. Let us focus on the observed parameters and characteristics of the stars.

\section{1. $\alpha$ Cyg}

On the Hertzsprung-Russell diagram, the massive A supergiant $\alpha$ Cyg is close to the HumphreysDavidson limit. I ts high luminosity (luminosity class Ia) manifests itself in large-scale macroturbulence [12], and the variability of brightness and velocity field in the extended shell. $\alpha$ Cyg is a prototype of the same type of hot variable supergiants, for which a low amplitude variability of brightness and radial velocity is typical. All the absorptions except $\mathrm{H} \alpha$ are symmetric in the star's spectrum (see an example in Fig. (1). Owing to its brightness and convenient location in the northern sky, the star is well studied and described in numerous papers. The main characteristics of the $\alpha$ Cyg spectrum 

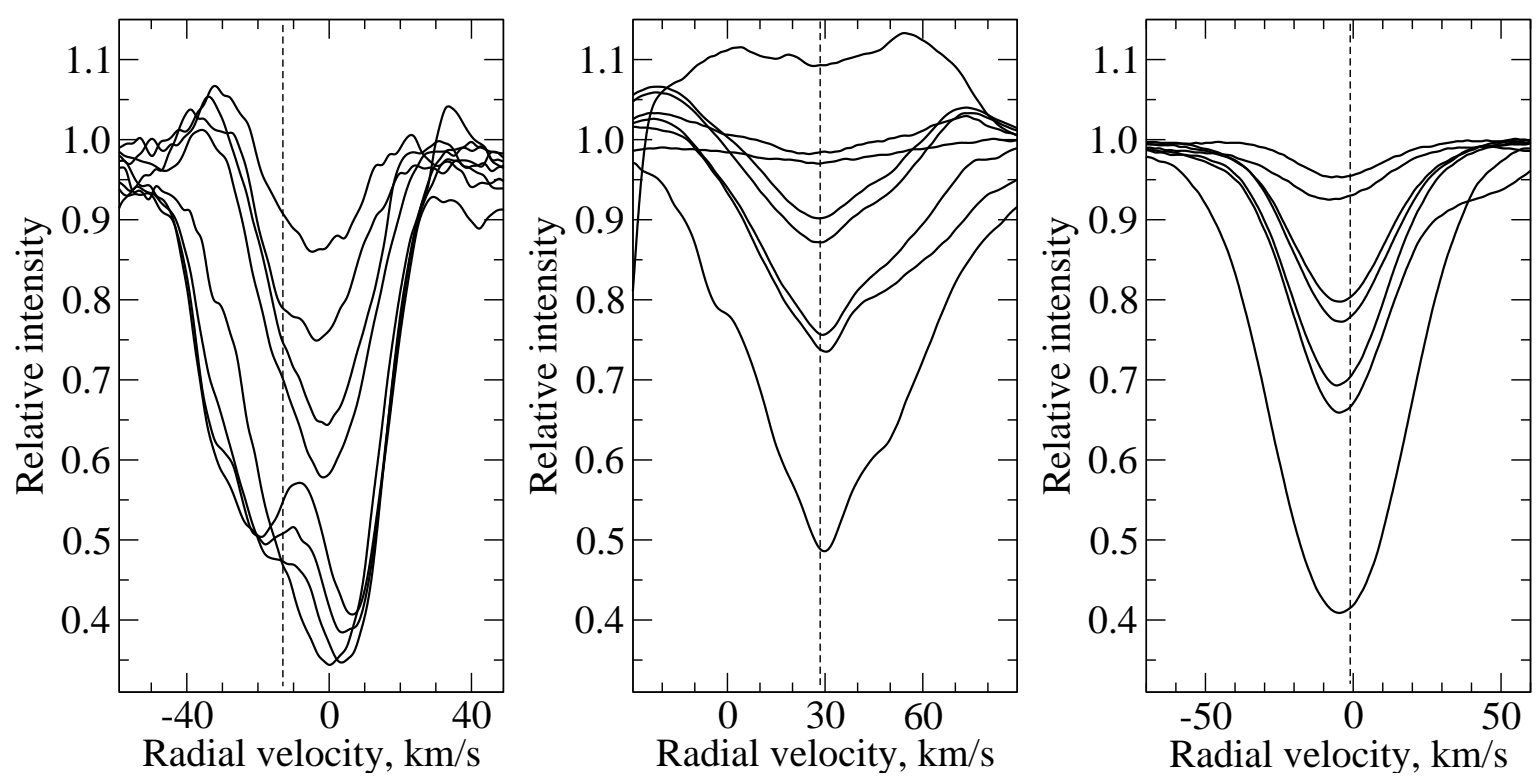

Figure 1. Line profiles of different intensity in the spectra of the studied stars. Left to right: $\mathrm{BD}+48^{\circ} 1220$ (top to bottom: Fe II 6433, 6248, 5535, 5363, 5169, 5018, 4924, 4523 ), 3 Pup (top to bottom: [O I] $6300 \AA$, Fe II 6369, 6084, 5284, $5535 \AA$; Ti II 4501, $4444 \AA$; Fe II $4233 \AA$ ), and $\alpha$ Cyg (top to bottom: Fe II $4433 \AA$; TiII 4444, $4501 \AA$; Fe II 5535, 5284, 5015, $6084 \AA$ ). The vertical dashed line denotes the systemic velocity $V_{\text {sys }}$ from [13, 14, 15] for BD $+48^{\circ} 1220,3$ Pup, and $\alpha$ Cyg respectively.

Table 1. General characteristics of the studied stars. The second column shows the spectral class and type of supergiant. Absolute $M_{V}$ magnitudes for $\alpha$ Cyg, 3 Pup, and BD $+48^{\circ} 1220$ from [16], [14, and 13 respectively, are given in the third column. The last two columns show the mid-exposure time in JD and the heliocentric radial velocity $V_{r}$ measured by numerous $(n>500)$ symmetric absorptions

\begin{tabular}{|c|c|c|c|c|c|c|c|}
\hline $\begin{array}{c}\text { Star } \\
\text { IR-source }\end{array}$ & $\begin{array}{c}S p \\
\text { status }\end{array}$ & $\begin{array}{l}M_{V} \\
\text { mag }\end{array}$ & $\begin{array}{l}V, \\
\text { mag }\end{array}$ & $\begin{array}{c}l, \\
\operatorname{deg}\end{array}$ & $\begin{array}{c}b, \\
\operatorname{deg}\end{array}$ & JD 2456000 & $\begin{array}{c}V_{r} \\
\mathrm{~km} \mathrm{~s}^{-1}\end{array}$ \\
\hline $\begin{array}{l}\mathrm{BD}+48^{\circ} 1220 \\
\text { IRAS } 05040+4820\end{array}$ & $\begin{array}{c}\text { A4 Ia } \\
\text { post-AGB }\end{array}$ & -5.0 & 9.74 & 159.7 & +04.8 & 581.4 & $-5.5 \pm 0.1$ \\
\hline $\begin{array}{l}3 \text { Pup } \\
\text { IRAS } 07418-2850\end{array}$ & $\begin{array}{l}\text { A2.7 Ib } \\
\text { peculiar }\end{array}$ & -5.5 & 3.93 & 244.4 & -02.5 & 579.6 & $+30.5 \pm 0.3$ \\
\hline $\begin{array}{l}\alpha \text { Cyg } \\
\text { IRC }+50337\end{array}$ & $\begin{array}{l}\text { A2 Iae } \\
\text { massive }\end{array}$ & -8.38 & 1.25 & 084.3 & +02.0 & 524.5 & $-5.2 \pm 0.1$ \\
\hline
\end{tabular}

and its variability are presented by Richardson et al. [17, who carried out a long-term spectral monitoring with a high spectral resolution in combination with Strömgren system photometry. However, despite the long-term observations of $\alpha \mathrm{Cyg}$, the nature of its variability remains unclear as long as the estimates of the star's mass and its brightness variability periods are not definitely stated [17, 18].

\subsection{Pup}

3 Pup was earlier considered as an object related to the stars evolving to a double nucleus planetary nebula [19. The reason for such a classification was its untypical for massive A supergiants featurean extended envelope around the star, the dust component of which appears in the IR excess [20]. According to the IRAS 21] data, 3 Pup is identified with the IR source IRAS 07418-2850. Only after determining the chemical composition of its atmosphere to be similar to Solar [22, a strong argument arose for 3 Pup to be a massive $\mathrm{A}[\mathrm{e}]$ supergiant. The $\mathrm{A}[\mathrm{e}]-\mathrm{B}[\mathrm{e}]$ phenomenon manifests itself in the combination of strong Balmer emission lines, and forbidden emissions [Fe II], [O I] with a considerable 
IR-flux excess associated with the hot dust shell. All these features are by definition typical for massive $\mathrm{B}[\mathrm{e}]$ supergiants [23] and are observed in the 3 Pup system.

Massive stars can be observed at the blue supergiant stage, or at the stage of evolving from the main sequence, or while evolving after the red supergiant stage. According to the review [24], supergiants with the $\mathrm{B}[\mathrm{e}]$ phenomenon in the optical spectrum can be viewed as objects evolving towards the red supergiant stage.

The inner gas shell influences the formation of peculiar double-peak emission details in the optical spectra of 3 Pup, which had been closely studied using the data on the spectral monitoring of this star carried out at the BTA during 1997-2008 [14. Moreover, in that paper we updated the spectral class $(\mathrm{A} 2.7 \pm 0.3 \mathrm{Ib})$ using the equivalent widths and profiles of $\mathrm{H} \delta$ and $\mathrm{H} \gamma$; and from the O I $7774 \AA$ triplet intensity, we estimated the luminosity $M_{V}=-5.5 \pm 0.3$.

As is evident from Table 2, which shows the depth and position measurements of the absorption and emission line components, the shell influences the majority of the lines; moreover, this influence gradually increases with the growth of line intensity and wavelength.

Regardless of numerous investigations of this star, which is an MK classification standard, the question of its binarity still remains open [25, 22. By the faintest photospheric absorptions, Chentsov et al. 14 found considerable (up to $7 \mathrm{~km} \mathrm{~s}^{-1}$ ) velocity changes from date to date, which indicate a companion star. The results of the monitoring by Chentsov et al. 14 are in overall agreement with the interpretation of Plets et al. 22] about 3 Pup being a star with an equatorial disk and a low mass companion.

Important data was obtained by Millour et al. 26] with the AMBER VLTI spectro-interferometer with millisecond spatial resolution, which allowed them to detect the dust and gas disks around 3 Pup. The second companion was not found; however, owing to the high spectral resolution of the equipment, the authors studied the velocity field of the object and concluded that there should be a second component which can form the Kepler disk and transfer the angular momentum to it.

\section{3. $B D+48^{\circ} 1220$}

The supergiant $\mathrm{BD}+48^{\circ} 1220 \quad\left(\mathrm{SAO} 40039, \mathrm{LSV}+48^{\circ} 26\right)$ is an optical component of the IRAS $05040+4820$ IR source. The star is rather close to the Galactic plane $(b=4.8)$, which can indicate its possible membership in the galactic disk population. Having multicolor photometric observations in the optical range for a sample of high luminosity stars, Fujii et al. [27] obtained for $\mathrm{BD}+48^{\circ} 1220$ the stellar magnitudes $B=10^{\mathrm{m}} 1, V=9.65$ and the A4 Ia spectral class. The high luminosity and the double-humped distribution of energy in the spectrum, indicating the presence of circumstellar matter which was ejected in the course of previous evolution, allow us to include $\mathrm{BD}+48^{\circ} 1220$ in the post-AGB star group.

Based on high spectral resolution $(R=60000)$ observations, Klochkova et al. 28] found the variability of the profiles and line positions. Afterwards, Klochkova et al. [13] thoroughly studied the optical spectrum of $\mathrm{BD}+48^{\circ} 1220$ in the wavelength range of 4500 to $6760 \AA$ and determined the basic parameters of the star and the chemical composition of its atmosphere. With the method of model atmospheres, they obtained the following parameters: effective temperature $T_{\text {eff }}=7900 \mathrm{~K}$, surface gravity $\log g=0.0$, and microturbulence velocity $\xi_{t}=6.0 \mathrm{~km} \mathrm{~s}^{-1}$. The metallicity of the star is almost similar to the solar one: $[\mathrm{Fe} / \mathrm{H}]_{\odot}=-0.10$. However, its chemical composition contains a set of peculiarities: a large helium abundance obtained from the $\mathrm{He} \mathrm{I} \lambda 5876 \AA$ absorption: $[\mathrm{He} / \mathrm{H}]_{\odot}=+1.04$ along with a considerable oxygen abundance $[\mathrm{O} / \mathrm{Fe}]_{\odot}=+0.72$. Also the modified abundances of light metals: $[\mathrm{Si} / \mathrm{Fe}]_{\odot}=+0.81,[\mathrm{Na} / \mathrm{Fe}]_{\odot}=+0.87$ with $[\mathrm{Mg} / \mathrm{Fe}]_{\odot}=-0.31$ and the ratio $[\mathrm{Na} / \mathrm{Mg}]_{\odot}=+1.18$ were found. A number of chemical composition peculiarities (a close-to-solar metallicity, carbon, lithium, and sodium overabundances) allow us to consider $\mathrm{BD}+48^{\circ} 1220$ as an analogue of the peculiar A supergiant V2324 Cyg, mentioned in the Introduction. The barium abundance in the BD $+48^{\circ} 1220$ atmosphere, however, is rather low: $[\mathrm{Ba} / \mathrm{Fe}]_{\odot}=-0.84$, whereas in the case of $\mathrm{V} 2324 \mathrm{Cyg}$, one can observe its slight overabundance: $[\mathrm{Ba} / \mathrm{Fe}]_{\odot}=+0.46$. 
The luminosity of the star and the peculiarities of its chemical composition (helium, lithium, silicon, sodium, and oxygen overabundances when $[\mathrm{C} / \mathrm{O}]<1$ ) allow us to refer $\mathrm{BD}+48^{\circ} 1220$ to the more massive post-AGB stars, which have ongoing HBB nuclear reactions at the base of the hot convective shell at the AGB stage. Moreover, we found distortions in the occurrence of chemical elements due to their selective separation into dust particles in the circumstellar envelope. The argument for the separation is a high zinc abundance, $[\mathrm{Zn} / \mathrm{Fe}]=+0.44$, and close values of the calcium and scandium deficiency. The above-mentioned peculiarities of the abundance of chemical elements in the atmosphere of $\mathrm{BD}+48^{\circ} 1220$ were later confirmed by the echelle spectra from the McDonald and Vainu Bappu [29] observatories. Altogether, the parameters $\left(M_{V} \approx-5^{\mathrm{m}}, V_{\mathrm{lsr}} \approx-20 \mathrm{~km} \mathrm{~s}^{-1}\right.$, metallicity $[\mathrm{Fe} / \mathrm{H}]_{\odot}=-0.10$, peculiarities of the optical spectrum and chemical abundance) confirm for BD $+48^{\circ} 1220$ the status of a He- and O-rich post-AGB star in the disk of the Galaxy with an initial mass of $M>4 M_{\odot}$. Note a rarely occurring feature of the chemical composition in the atmosphere of $\mathrm{BD}+48^{\circ} 1220$ - a reliably estimated nickel overabundance.

Thus, for producing the atlas, we chose three A supergiants with different masses and evolutionary stages. The observed characteristics of these stars differ fundamentally, which ensures the significance of comparing their optical spectra.

\section{Spectral data}

The spectra of all the objects that we used for producing the atlas were obtained in the Nasmyth focus of the 6-m telescope. The echelle spectrograph NES [30] in combination with a CCD of $2048 \times$ 4608 elements and an image slicer [31] provides the spectral resolution $R=60000$. The Julian date for the mid-exposure time is given in the next-to-last column of Table 1.

The extraction of data from two-dimensional echelle images was conducted with the modified [32] ECHELLE context of ESO-MIDAS in the Linux operating system. Cosmic ray hit were removed by median averaging of two spectra obtained successively one after another. Wavelength calibration was done using the Th-Ar lamp with a hollow cathode. Further reduction of one-dimensional spectra, including continuum normalization, photometric and position measurements, was performed using the computer code DECH 20T [33] version 7.3.23. To increase the measurement accuracy of the intensity and spectral line positions by comparing the observed spectra with the corresponding synthetic ones, unblended lines were selected. The zero-point position of every spectrogram was determined with the standard technique - matching with the positions of telluric absorptions and ionospheric emissions which can be observed along with the spectrum of the object. The accuracy of single-line velocity measurements in the spectra obtained by NES is higher than $1 \mathrm{~km} \mathrm{~s}^{-1}$ [13].

\section{Atlas description}

The production of spectral atlases is particularly important in connection with the improvement in the quality of observational data due to the use of up-to-date high resolution spectrographs that record spectra on low-noise CCDs. Furthermore, at present there is a possibility of presenting the graphical information from the atlases and the detailed tables with line identifications digitally, which provides users with unlimited access to this observational data.

The spectra of the stars under study are presented in the atlas in the form of diagrams (see Figs. 3 10). These figures show the dependences of the residual intensity $r$ on the laboratory wavelength, they are arranged one below the other in the same order as the objects in Table 1. To reduce the observed spectra to the laboratory wavelengths, we determined the radial velocities $V_{r}$ of the stars at the moment of their observation; the lines of low and moderate intensity without any evident distortions were selected for this procedure. It should be emphasized that we have not found differential shifts of these lines in the spectra of the studied stars. When changing the wavelengths to the laboratory ones, this allowed us to apply the average values $V_{r}=-5.5,+30.5,-5.2 \mathrm{~km} \mathrm{~s}^{-1}$ for BD $+48^{\circ} 1220,3 \mathrm{Pup}$, and $\alpha$ Cyg respectively.

For each star the $r(\lambda)$ values of individual echelle orders were combined into one array which was then divided into equal fragments of $100 \AA$. Each fragment of the spectral atlas shows the identification 
of several lines. For the identification of the spectral details, we used the results from the papers [14, 13] and also the data from VALD (see [34] and the references to the previous publications regarding the earlier versions).

The results of line identification and measurement are shown in Table 2, The first two columns give the name of the chemical element, the multiplet number, and the used laboratory wavelength according to [34. The following columns of this table show, for each star, the central residual intensities of the absorptions $r$ and the heliocentric radial velocities $V_{r}$ in $\mathrm{km} \mathrm{s}^{-1}$ measured from the absorption cores of single lines or from distinct components of blends. We use central residual intensities instead of equivalent widths for ease of comparison of tabular and graphical data. The horizontal lines in the table separate single lines and blends. The entire fragments of the spectra and Table 2 can be found at http://www.sao.ru/hq/ssl/Deneb/atlas.html. Let us further consider some features of the spectra of the stars under study.

\section{1. $\alpha$ Cyg}

Atlas [35] presents the fragments of the $\alpha$ Cyg spectrum in a limited wavelength region $(3826-5212 \AA)$ with a resolution similar to ours but with a higher signal-to-noise ratio $(S / N=300-1400)$. All the absorptions from this atlas down to the faintest ones with residual intensities $r<0.995$ are detectable in our spectrum. Residual intensities do not differ systematically. Chentsov et al. [36] published a spectral atlas for four stars in a wide range of wavelengths 3055-4520 , including the UV radiation reaching the Earth's surface. In atlas [36], the spectra of the well-studied stars with similar temperatures but different luminosities ( $\beta$ Ori, $\alpha \mathrm{Lyr}$, and $\alpha \mathrm{Cyg}$ ) are compared with the spectrum of a low metallicity A supergiant KS Per, the atmosphere of which is hydrogen-poor, $\mathrm{H} / \mathrm{He}=3 \times 10^{-5}$.

The shortwave spectral region of $\alpha \mathrm{Cyg}$ studied in this paper is comparable with the longwave spectral region in atlas [36. The spectra from both atlases were obtained with the same spectrograph NES with identical spectral resolution, which makes it possible to investigate the spectrum of $\alpha$ Cyg in a wide wavelength range 3055-6720 $\AA$.

\section{2. $B D+48^{\circ} 1220$}

The optical spectrum of $\mathrm{BD}+48^{\circ} 1220$ differs from the one of $\alpha$ Cyg by the presence of numerous lines with asymmetric profiles distorted by emission components. This difference is well illustrated by the profiles on the left and right sides of Fig. 1. The $\mathrm{H} \alpha$ line in the $\mathrm{BD}+48^{\circ} 1220$ spectrum has a complex two-component emission profile, for which time variability is typical, as follows from the comparison of the profile in Fig. 2 with the data from [13]. Let us note particularly that over the decade that passed since we obtained the spectra used in [13], the ratio of the intensities of the shortwave and longwave emission components of $\mathrm{H} \alpha$ reversed. Variable emission components are also observed in the metal lines Si II, Fe I, Fe II. This variability is due to the non-sphericity of the circumstellar shell, which is confirmed by investigations of the polarization of the light of the star [37].

For a star with an effective temperature lower than $8000 \mathrm{~K}$, the presence of a strong absorption of neutral helium HeI $\lambda 5876 \AA$ with an equivalent width of $W_{\lambda}(5875)=75 \mathrm{~m} \AA$ is unexpected. The absorption character of the line shows its photospheric nature; this is also proved by the fact that the radial velocity corresponding to the line position agrees with the velocity measured from other weak absorptions (see Table 2).

The presence of weak absorptions identified with well-known diffuse interstellar bands (DIBs) is also a peculiarity of the $\mathrm{BD}+48^{\circ} 1220$ spectrum. They are listed in Table 2, which also gives their equivalent widths and heliocentric velocities corresponding to the positions of these details. The average heliocentric velocity $V_{r}$ according to the interstellar bands agrees with the velocity measured from the main component of the Na D lines: $V_{r}(\mathrm{DIBs}) \approx-2 \mathrm{~km} \mathrm{~s}^{-1}$. 


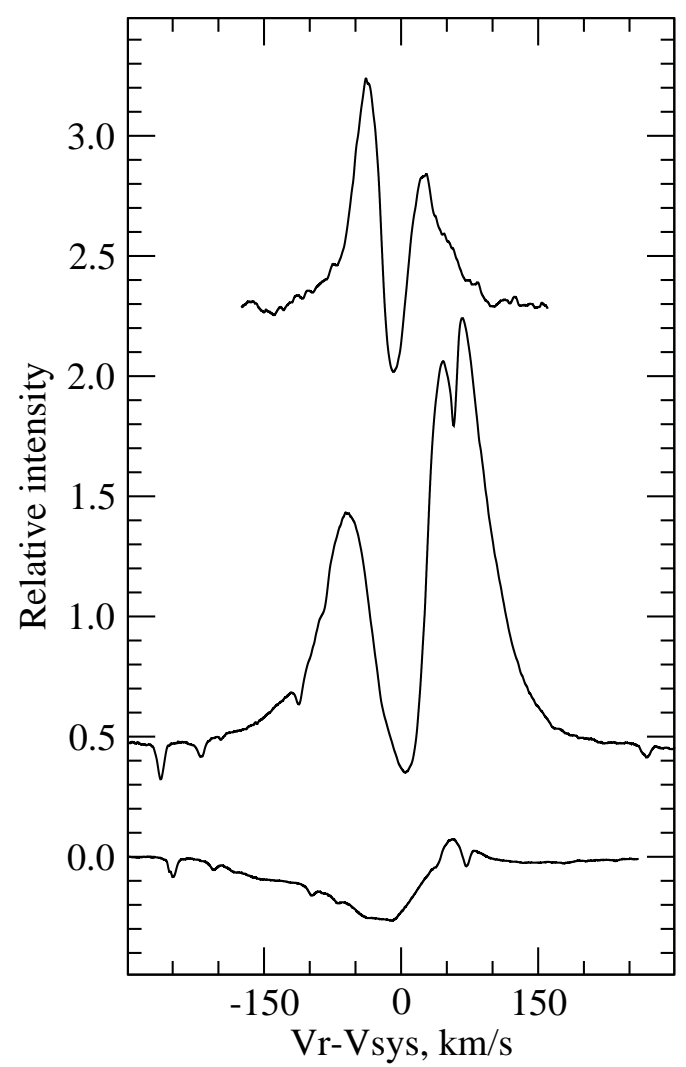

Figure 2. Central parts of $\mathrm{H} \alpha$ profiles in the spectra of the supergiants under study (top to bottom): $\mathrm{BD}+48^{\circ} 1220,3 \mathrm{Pup}$ and $\alpha \mathrm{Cyg}$. The $\mathrm{H} \alpha$ profiles for $3 \mathrm{Pup}$ and BD $+48^{\circ} 1220$ are shifted upwards relative to the profile of the $\alpha$ Cyg spectrum by 0.5 and 2.3 respectively. The difference of the heliocentric and systemic velocities $V_{r}-V_{\text {sys }}$ in $\mathrm{km} \mathrm{s}^{-1}$ is given for each star on the horizontal axis. Telluric details are not removed.

\subsection{Pup}

The characteristic feature of the 3 Pup spectrum - the double-peak emission details of numerous line profiles (mainly of Fe II ions) - is well reproduced in our atlas. In particular, such specific profiles with uplifted wings are presented in the central part of Fig. 1 and in Fig. 10. The $\mathrm{H} \alpha$ emission line in the 3 Pup spectrum has a complex split profile with the red component prevailing (Fig. 2).

A useful comparison of the line profiles of different intensities in the spectra of $3 \mathrm{Pup}$ and $\alpha \mathrm{Cyg}$ was conducted in [14. A comparison of the details shows that in the $\alpha$ Cyg spectrum all the lines are symmetric and differ in depth only. They are formed near the photosphere. Only the Mg II $4481 \AA$ line can be considered photospheric in 3 Pup; as for the Fe II lines, here the shell contribution is apparent which gives the profiles a specific form: the wings are uplifted by emissions, and the core is sharpened by a depression. The depression is noticeable even in the absorptions with the residual intensity of $r \approx 0.2$, and it grows with the line strength. A comparison of the line profiles and their parameters in the spectra of $3 \mathrm{Pup}$ and $\alpha \mathrm{Cyg}$, obtained with one and the same spectral resolution, showed that the manifestation of the shell in 3 Pup is not limited to certain lines. It grows gradually with the increase of the intensity and wavelength of the line. The central depths of absorptions in the spectra of 3 Pup and $\alpha$ Cyg are compared in Fig. 1 of [14].

The anomalies of the line profiles in the 3 Pup spectrum naturally affect the measurements of radial velocities. The $V_{r}(r)$ dependence derived from the measurements of lines in several spectra obtained on different dates is variable in time. However, for the forbidden emissions and the narrow shell cores of absorptions of the strong lines of the iron-group ions, the velocity variations in time are minimal, and the velocities derived from such lines are similar to each other. The average velocity for the mentioned 
lines can be assigned as the radial velocity of the whole system: $V_{\mathrm{sys}}=28.5 \pm 0.5 \mathrm{~km} \mathrm{~s}^{-1}$ [14]. The average velocity $V_{r}$ measured from the interstellar DIBs is $V_{r}=+28.55 \mathrm{~km} \mathrm{~s}^{-1}$.

\section{Conclusions}

Using high spectral resolution observations, we compiled a spectral atlas in the wavelength range of 3920-6720 A for three A supergiants with different evolutionary status: a massive supergiant $\alpha$ Cyg, a peculiar supergiant with a circumstellar disk 3 Pup, and a post-AGB star BD $+48^{\circ} 1220$. A comparison of their spectra leads to the conclusion that the determination of the evolutionary status of supergiants in the galactic field is a sophisticated problem, as the stars can be similar in spectral and luminosity classes but significantly different in age, mass, and observed on fundamentally different stages of evolution. One and the same region in the Hertzsprung-Russell diagram can be occupied by postAGB stars evolving from the AGB stage to a planetary nebula, and massive supergiants evolving from the main sequence to the red supergiant stage. One spectacular example is the post-AGB star $\mathrm{BD}+48^{\circ} 1220$ and the massive supergiant 3 Pup with similar spectral features.

Practical experience with the spectra of stars of different types suggests that for definite conclusions on the evolutionary status of HLSs, the entire set of basic stellar parameters is required; foremost, the luminosity, the velocity field in the atmosphere, and the detailed chemical composition. High spectral resolution spectroscopy, which provides the data required for determining these parameters, is the main approach in stellar astrophysics. Spectropolarimetry and high spatial resolution observations also provide the necessary information.

Acknowledgements. The investigation was carried out with the support of the Russian Foundation for Basic Research (projects 12-07-00739a, 14-02-00291a). Observations with the 6-m BTA telescope are conducted with the financial support of the Ministry of Education and Science of the Russian Federation (Agreement No. 14.619.21.0004, project identifier RFMEFI61914X0004). This research has made use of the SIMBAD and ADS databases.

\section{References}

1. P. Massey, New Astron. Rev. 57, 14 (2013).

2. R.M. Humphreys, K. Davidson, S. Grammer, et al., Astrophys. J. 773, 46 (2013).

3. S. Kwok, Ann. Rev. Astron. Astrophys. 31, 63 (1993).

4. V.G. Klochkova, E.L. Chentsov, V.E. Panchuk, Mon. Not. R. Astron. Soc. 292, 19 (1997).

5. V.G. Klochkova, M.V. Yushkin, E.L. Chentsov, V.E. Panchuk, Astron. Rep. 46, 139 (2002).

6. V.G. Klochkova and E.L. Chentsov, Astrophys. Bull. 63, 112 (2008).

7. P. Ventura, R. Carini, and F.D. D'Antona, Mon. Not. R. Astron. Soc. 415, 3865 (2011).

8. B.M. Lewis, Astrophys. J. 338, 234 (1989).

9. P. Garcia-Lario, A. Manchado, S.R. Suso, and S.R. Pottash, Astron and Astrophys. Suppl.82, 497 (1990).

10. V.P. Arkhipova, N.P. Ikonnikova, R.I. Noskova, and G.V. Sokol, Astron. Let. 26, 609 (2000).

11. D. Hoffleit and C. Jaschek, The Bright Star Catalogue, 4th ed. (Yale University Observatory, New Haven, 1982).

15. B. Boer, C. de Jager, and H. Nieuwenhuijzen, Astron and Astrophys.195, 218 (1988).

14. V.G. Klochkova, E.L. Chentsov, N.S. Tavolzhanskaya, and V.E. Panchuk, Astron. Rep. 51, 642 (2007).

13. E.L. Chentsov, V.G. Klochkova, and A.S. Miroshnichenko, Astrophys. Bull. 65, 150 (2010).

16. A. Kaufer, O. Stahl, B. Wolf, et al., Astron and Astrophys.305, 887 (1996).

12. F. Schiller and N. Przybilla, Astron and Astrophys.479, 849 (2008).

17. N.D. Richardson, N.D. Morrison, E.E. Kryukova, and S.J. Adelman, Astron. J. 141, 17 (2011).

18. H. Saio, C. Georgy, and G. Meynet, Mon. Not. R. Astron. Soc. 433, 1246 (2013). 
19. M. Jura and S.G. Kleinmann, Astrophys. J. 341, 359 (1989).

20. A. Meilland, S. Kanaan, M. Borges Fernandes, et al., Astron and Astrophys.512, A73 (2010).

21. N.R. Trams, L.B.F.M. Waters, H.J.G.L. M. Lamers, et al., Astron and Astrophys. Suppl.87, 361 (1991).

22. H. Plets, C. Waelkens, and N. R. Trams, Astron and Astrophys.293, 363 (1995).

23. H.J.G.L.M. Lamers, F.J. Zickgraf, D. de Winter, et al., Astron and Astrophys.340, 117 (1998).

24. W.J. de Wit, R. Oudmaijer, and J.S. Vink, Advances in Astronomy 2014, id. 270848 (2014).

25. L.O. Loden, The Messenger, No. 68, 26 (1992).

26. F. Millour, A. Meilland, O. Chesneau, et al., Astron and Astrophys.526, A107 (2011).

27. T. Fujii, Y. Nakada, and M. Parthasarathy, Astron and Astrophys.385, 884 (2002).

28. V.G. Klochkova, E.L. Chentsov, V.E. Panchuk, and M.V. Yushkin, IBVS, No. 584, (2004).

29. S.S. Rao, G. Pandey, D.L. Lambert, and S. Giridhar, Astrophys. J. 737, L7 (2011).

30. V. Panchuk, V. Klochkova, M. Yushkin, and I. Najdenov, J. Optical Technology 76, 87 (2009).

31. V. Panchuk, M. Yushkin, and I. Najdenov, Preprint No. 179, SAO RAS (Spec. Astrophys. Obs. RAS, 2003).

32. M. Yushkin and V. Klochkova, Preprint No. 206, (Special Astrophysical Observatory, Nizhnii Arkhyz, 2005).

33. G.A. Galazutdinov, Preprint No. 92, SAO (Special Astrophysical Observatory, Nizhnii Arkhyz, 1992).

34. F. Kupka, N. Piskunov, T.A. Ryabchikova, et al., Astron and Astrophys. Suppl.138, 119 (1999).

35. B. Albayrak, A.F. Gulliver, S.J. Adelman, et al., Astron and Astrophys.400, 1043 (2003).

36. E.L. Chentsov, V.G. Klochkova, T. Kipper, et. al., Astrophys. Bull. 66, 466 (2011).

37. M. Parthasarathy, S. K. Jain, and G. Sarkar, Astron. J. 129, 2451 (2005). 

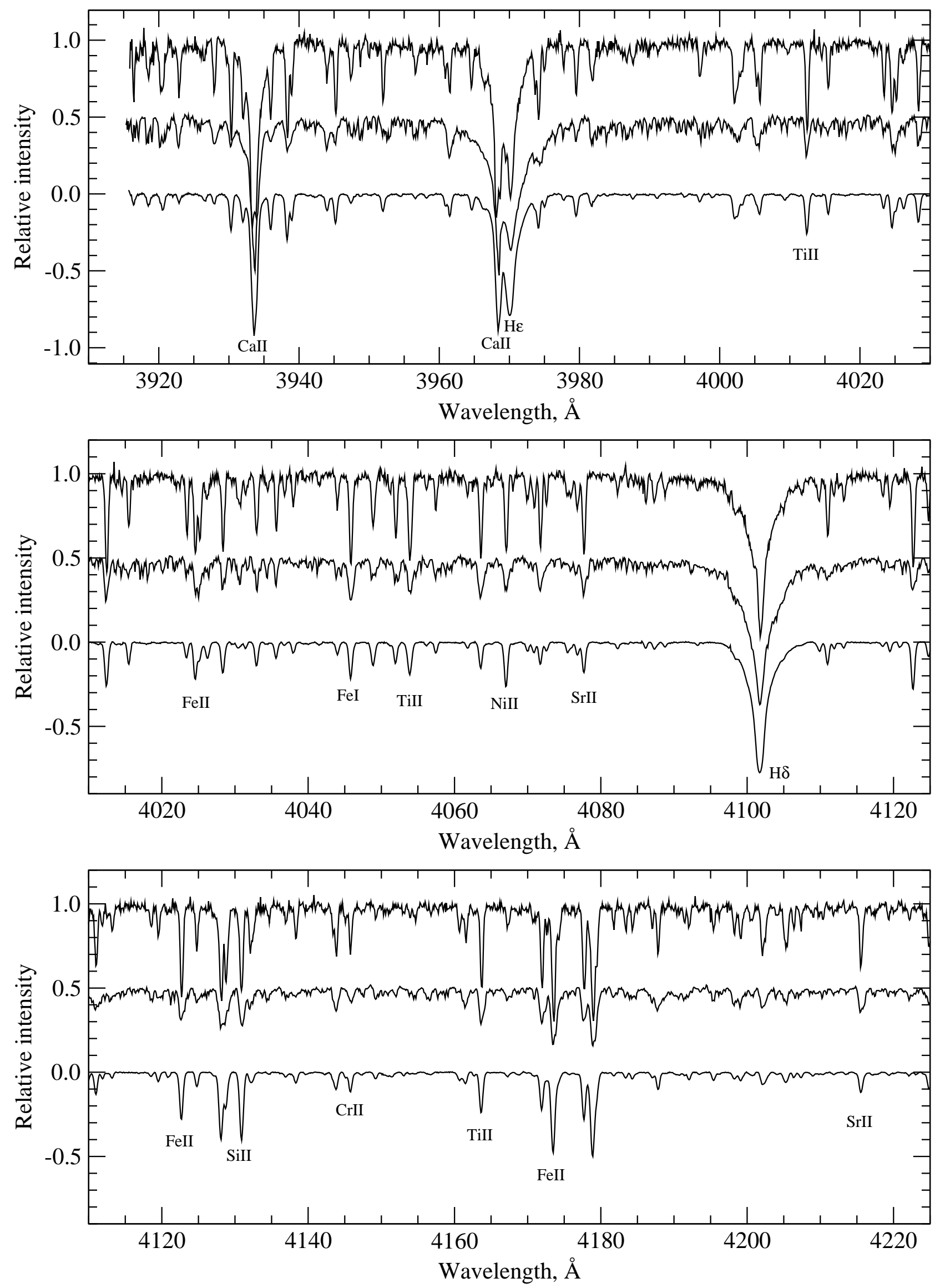

Figure 3. Three shortwave fragments from the atlas in the wavelength range of 3920-4220 $\AA$. Hereafter, the spectrum fragments are arranged top to bottom: $\mathrm{BD}+48^{\circ} 1220,3 \mathrm{Pup}, \alpha \mathrm{Cyg}$, shifted along the vertical axis relative to each other. 

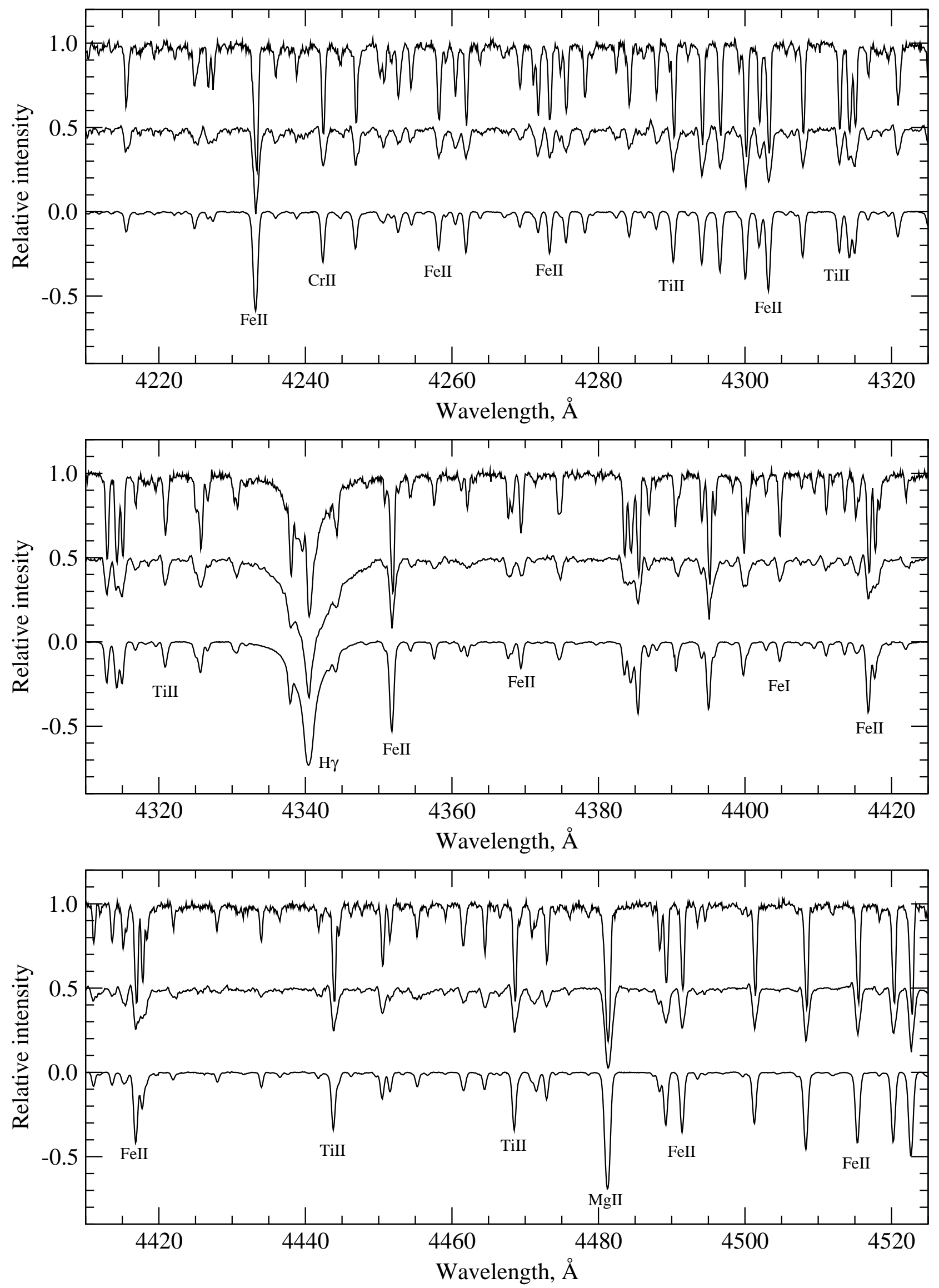

Figure 4. 

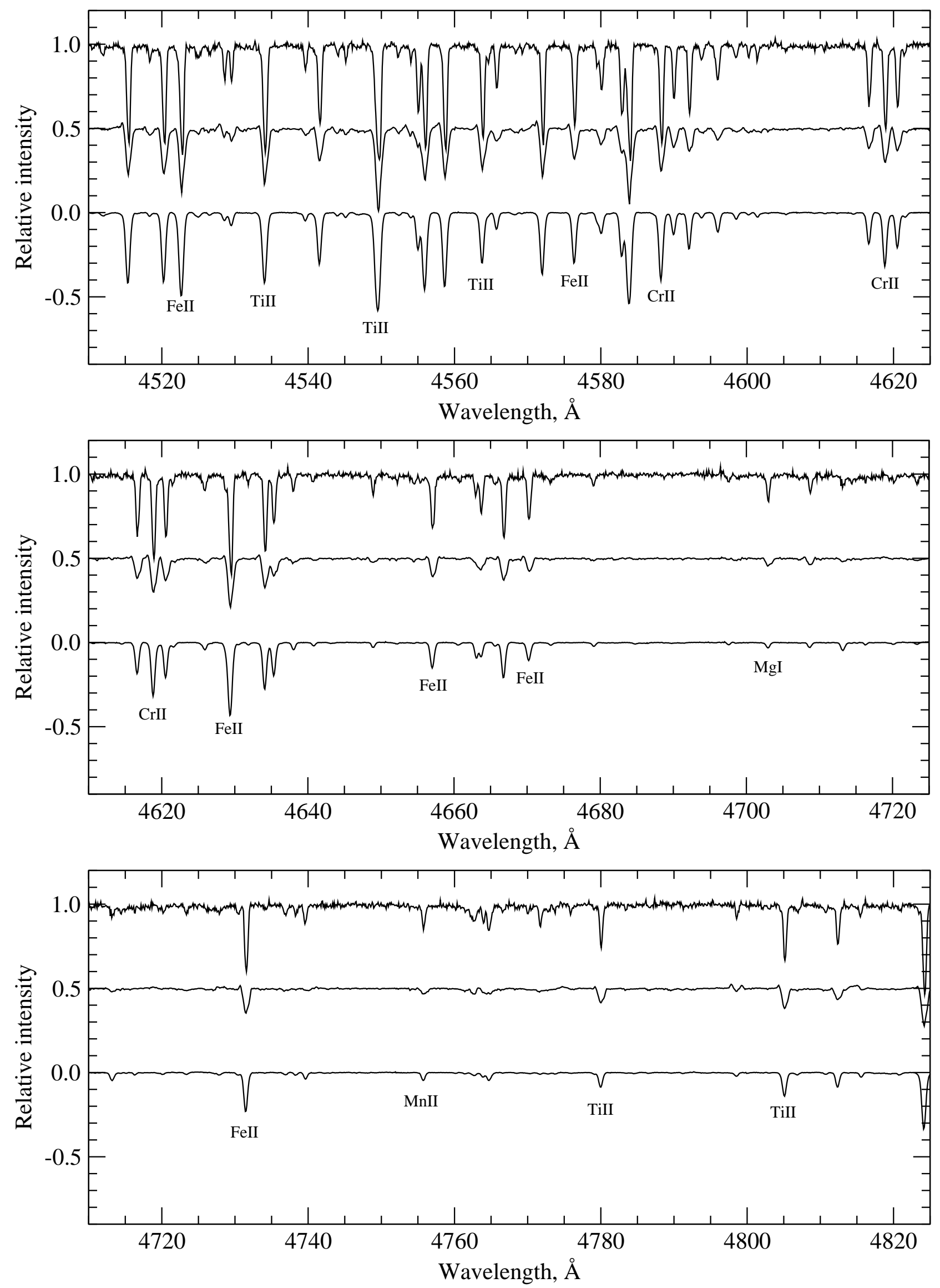

Figure 5. 

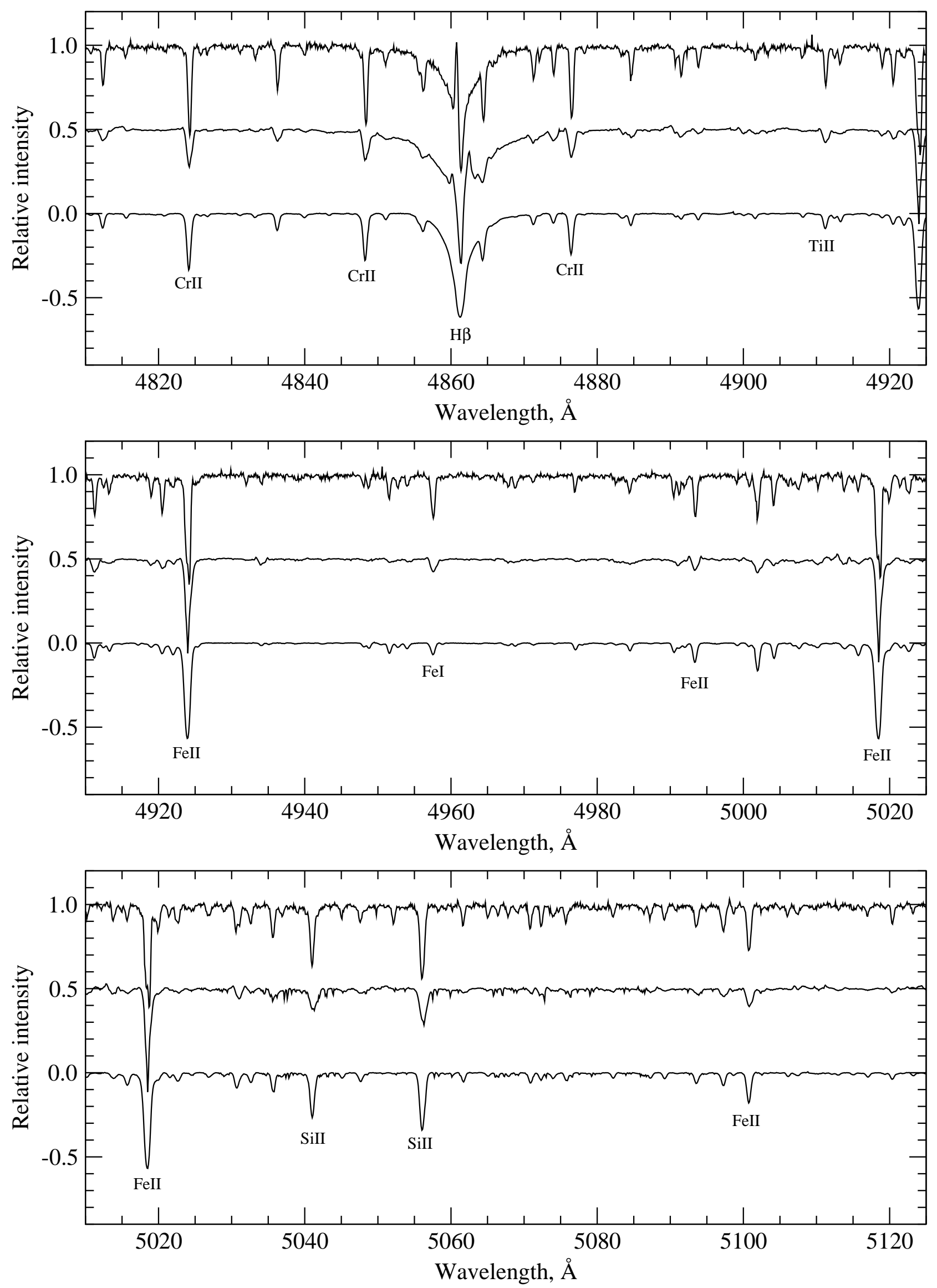

Figure 6. 

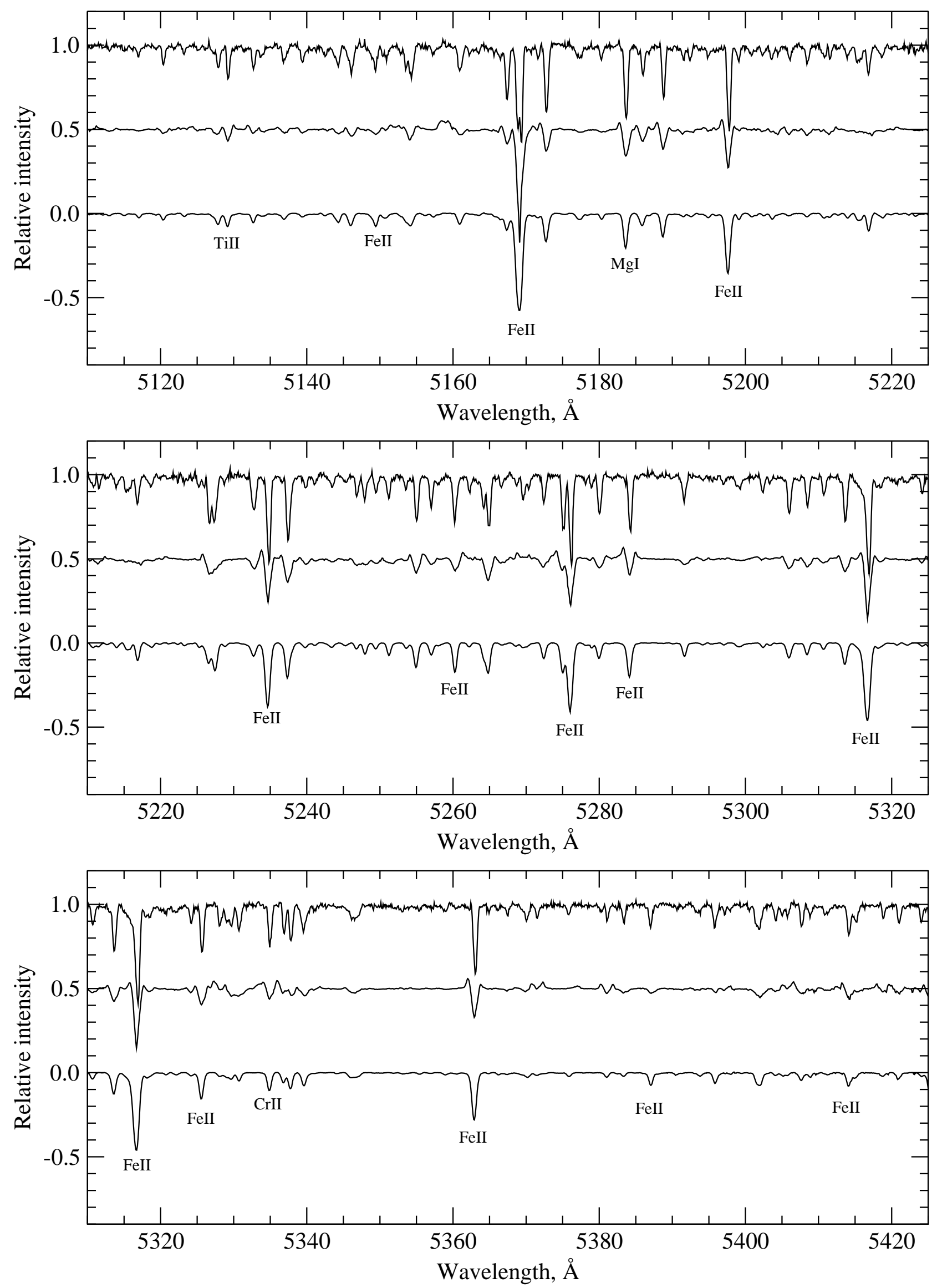

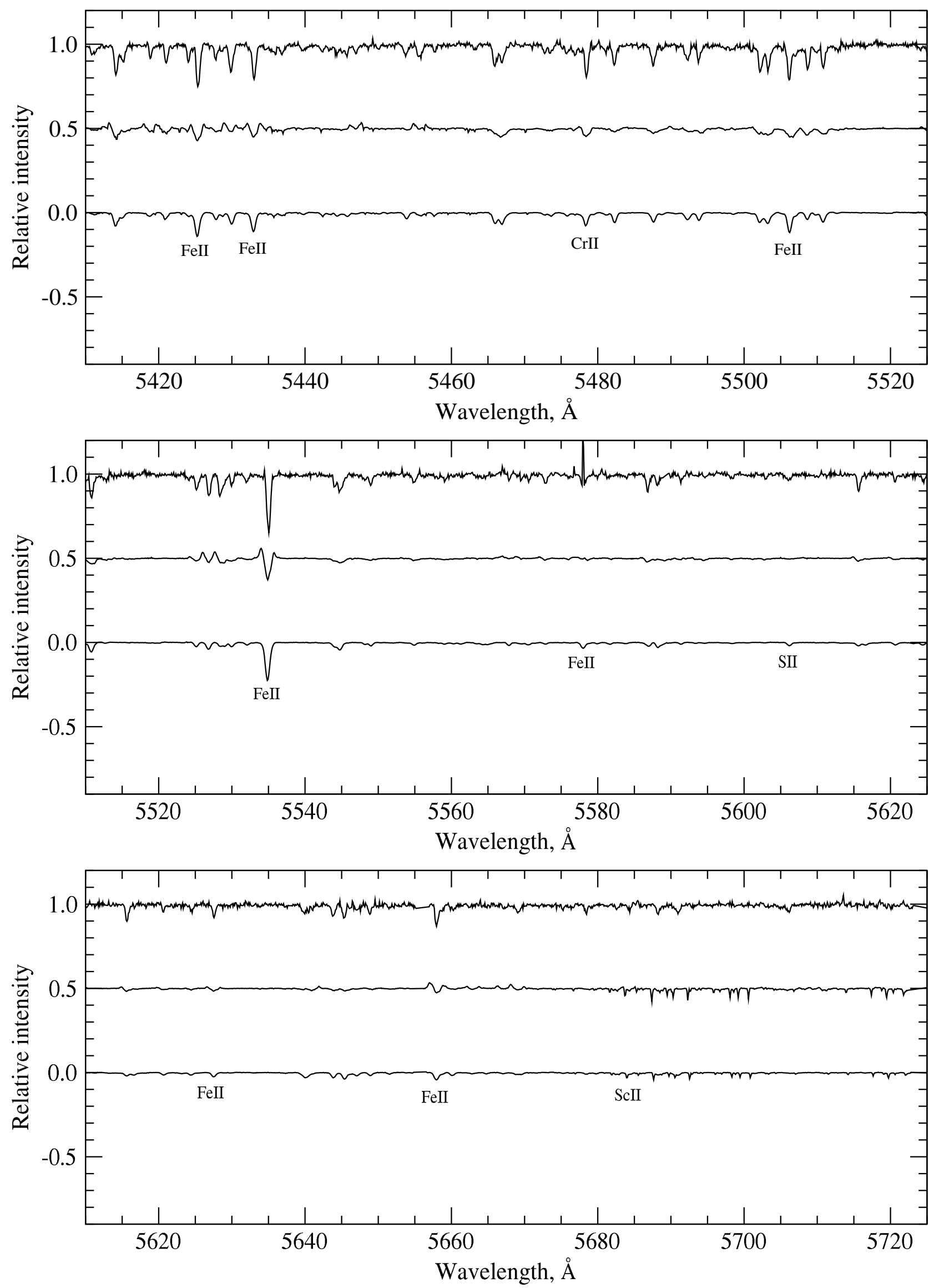

Figure 7. 

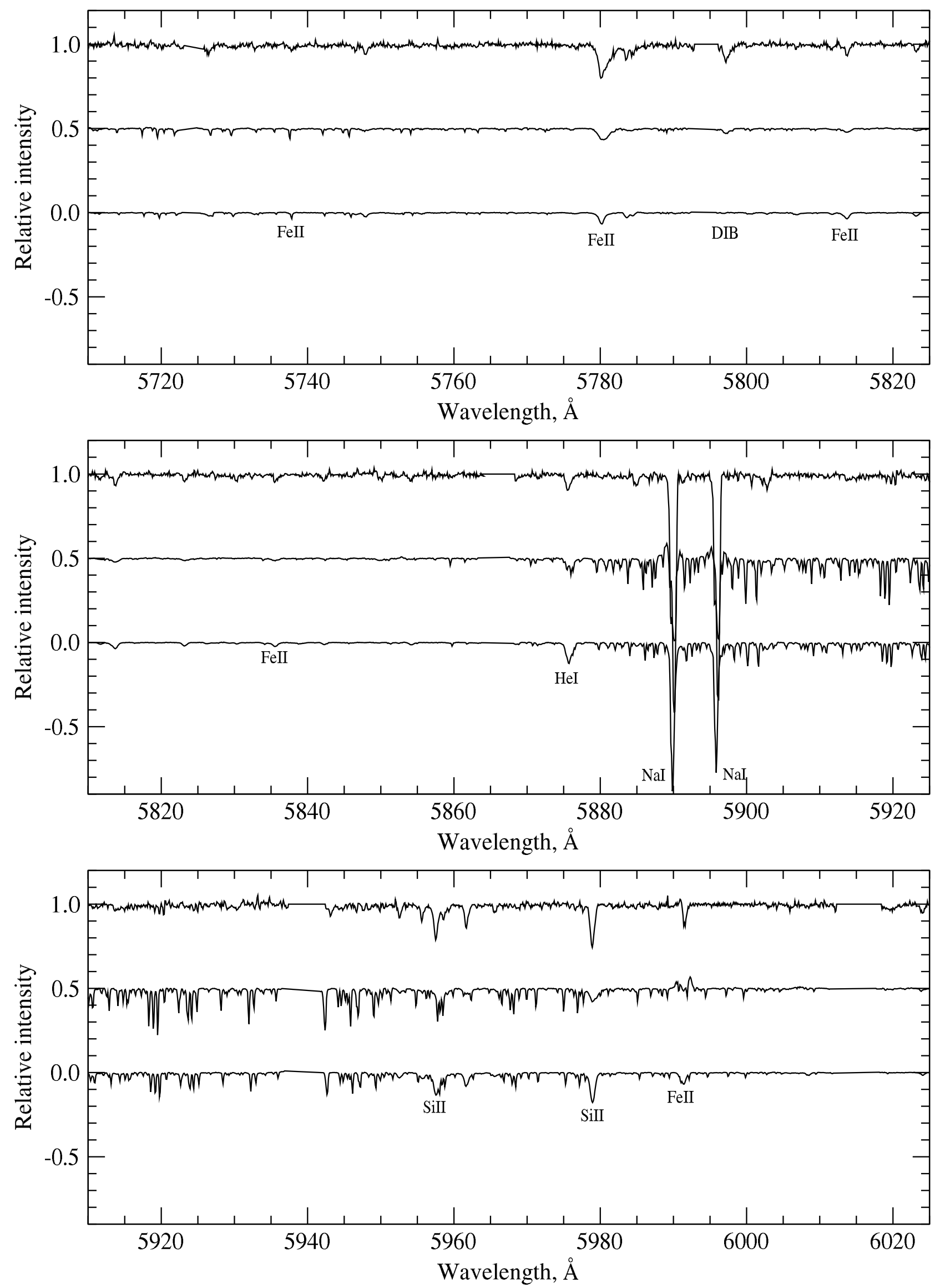

Figure 8. 

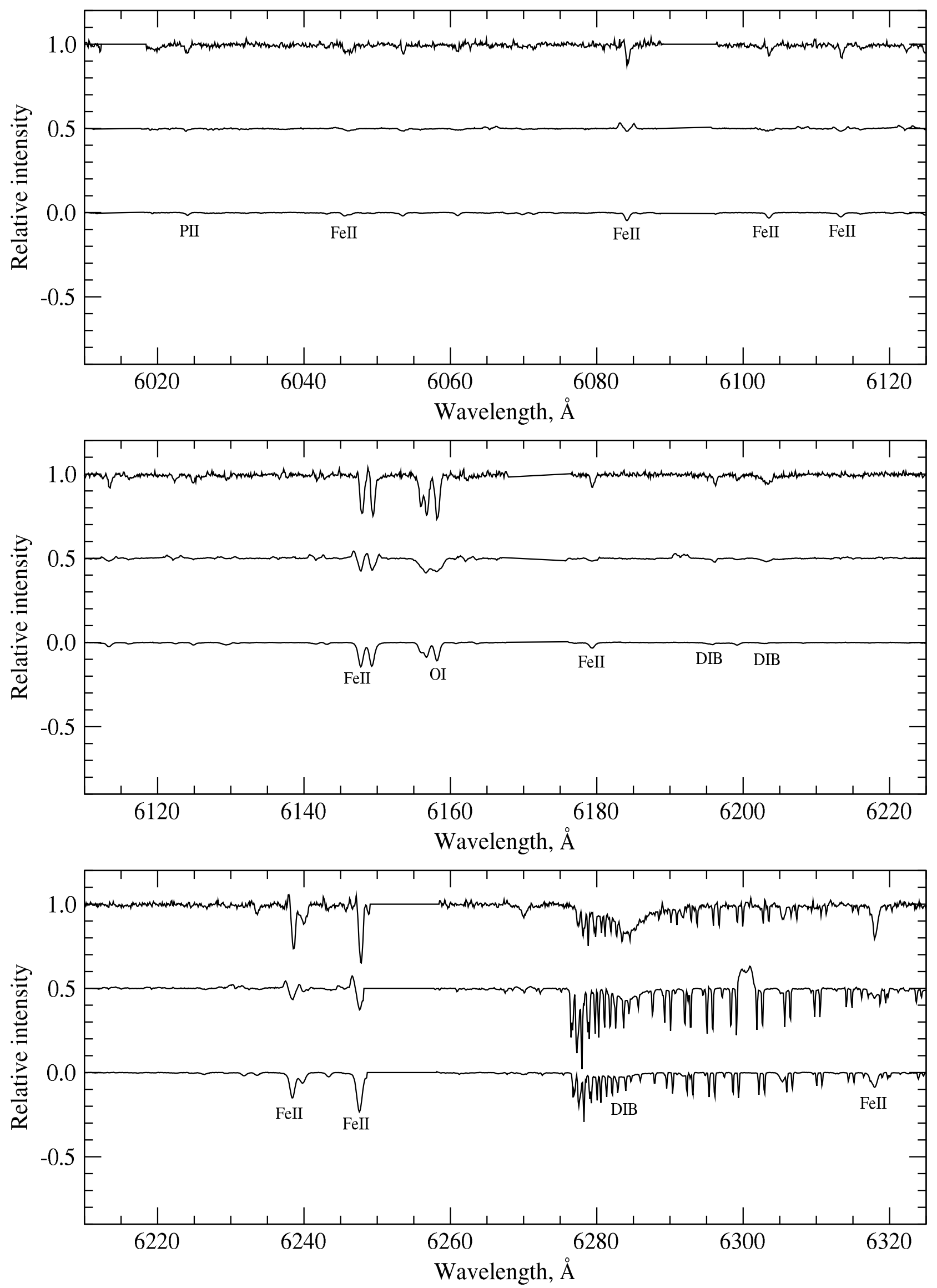

Figure 9. The atlas fragments in the wavelength range of $6120-6520 \AA$. 

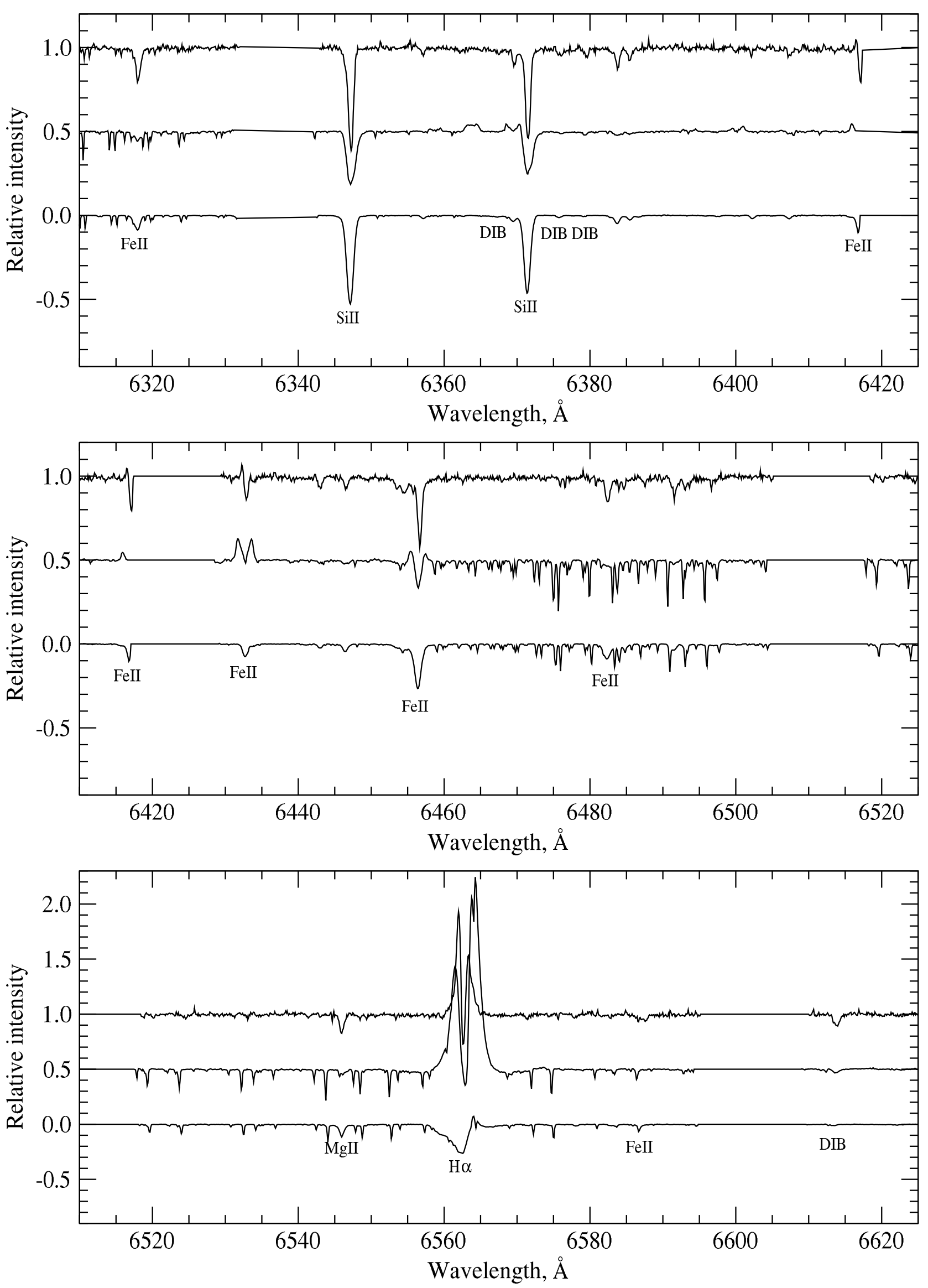

Figure 10. Three longwave fragments from the atlas. The spectrum are arranged top to bottom: $\mathrm{BD}+48^{\circ} 1220,3 \mathrm{Pup}, \alpha \mathrm{Cyg}$. The telluric details are not removed 
Table 2: Residual intensities $r$ and heliocentric radial velocities $V_{r}\left(\mathrm{~km} \mathrm{~s}^{-1}\right)$ for the spectral features in the high resolution spectra of $\alpha \mathrm{Cyg}, 3 \mathrm{Pup}$, and $\mathrm{BD}+48^{\circ} 1220$. The horizontal lines separate single lines or unresolved blends. Uncertain measurements are marked with a colon

\begin{tabular}{|c|c|c|c|c|c|c|c|}
\hline \multirow[t]{2}{*}{ Ident } & \multirow[t]{2}{*}{$\lambda$} & \multicolumn{2}{|c|}{$\alpha \mathrm{Cyg}$} & \multicolumn{2}{|c|}{$3 \mathrm{Pup}$} & \multicolumn{2}{|c|}{ IRAS 05040} \\
\hline & & $r$ & $V_{r}(\mathrm{~km} / \mathrm{s})$ & $r$ & $V_{r}(\mathrm{~km} / \mathrm{s})$ & $r$ & $V_{r}(\mathrm{~km} / \mathrm{s})$ \\
\hline VII(10) & 3916.41 & 0.93 & -4.66 & & & $0.65:$ & -11.8: \\
\hline $\begin{array}{l}\text { FeII(191) } \\
\text { CII }\end{array}$ & $\begin{array}{l}3918.53 \\
3918.97\end{array}$ & 0.92 & -4.11 & & & $0.77:$ & $-12.4:$ \\
\hline $\begin{array}{l}\text { FeI(4) } \\
\text { FeII } \\
\text { CII }(4)\end{array}$ & $\begin{array}{l}3920.26 \\
3920.64 \\
3920.68\end{array}$ & 0.89 & -8.58 & & & 0.68: & \\
\hline FeII & 3922.00 & 0.98 & -5.74 & & & & \\
\hline $\mathrm{FeI}(4)$ & 3922.91 & 0.94 & -6.55 & 0.8 & 17.4 & 0.62 & -13.2 \\
\hline $\mathrm{HeI}(58)$ & 3926.54 & 0.95 & -6.34 & & & $0.87:$ & \\
\hline $\mathrm{FeI}(4)$ & 3927.92 & 0.94 & -8.03 & 0.83 & 28: & 0.65 & -11.6 \\
\hline $\begin{array}{l}\mathrm{VII}(10) \\
\mathrm{FeI}(4)\end{array}$ & $\begin{array}{l}3929.72 \\
3930.30\end{array}$ & 0.75 & -5.43 & $0.81:$ & $22:$ & $\begin{array}{l}0.85: \\
0.35\end{array}$ & $\begin{array}{l}-13: \\
-8.5\end{array}$ \\
\hline $\operatorname{TiII}(34)$ & 3932.01 & 0.81 & -4.56 & & & $0.49:$ & $-9.8:$ \\
\hline $\begin{array}{l}\text { FeI } \\
\text { SII } \\
\mathrm{CaII}(1) \\
\mathrm{CaII}(1) \\
\mathrm{ZrII} \\
\mathrm{ZrII}\end{array}$ & $\begin{array}{l}3932.63 \\
3933.26 \\
3933.66 \\
3933.66 \\
3934.09 \\
3934.79\end{array}$ & 0.08 & -6.42 & 0.43 & 24 & & $\begin{array}{l}-37 \\
-12\end{array}$ \\
\hline $\begin{array}{l}\mathrm{FeI}(362) \\
\mathrm{FeII}(173) \\
\mathrm{CoI}(32)\end{array}$ & $\begin{array}{l}3935.82 \\
3935.96 \\
3935.97\end{array}$ & 0.77 & -6.38 & $0.84:$ & 23.8: & 0.48 & $-12.7:$ \\
\hline $\begin{array}{l}\mathrm{FeII}(3) \\
\operatorname{MgI}(18) \\
\mathrm{FeII}(190)\end{array}$ & $\begin{array}{l}3938.29 \\
3938.40 \\
3938.97\end{array}$ & $\begin{array}{c}0.7 \\
0.83 \\
\end{array}$ & $\begin{array}{l}-4.88 \\
-8.59 \\
\end{array}$ & 0.79 & $28.5:$ & $\begin{array}{l}0.36 \\
0.65 \\
\end{array}$ & $\begin{array}{r}-7.8 \\
-14.8 \\
\end{array}$ \\
\hline $\begin{array}{l}\text { FeI } \\
\text { FeI }\end{array}$ & $\begin{array}{l}3940.88 \\
3941.28\end{array}$ & 0.98 & -7.27 & & & & \\
\hline $\begin{array}{l}\text { NiI } \\
\text { FeI }(364)\end{array}$ & $\begin{array}{l}3941.84 \\
3942.44\end{array}$ & 0.98 & -5.46 & & & 0.93: & \\
\hline $\begin{array}{l}\text { AlI(1) } \\
\text { NiI } \\
\text { FeII } \\
\text { FeI } \\
\text { FeII(3) } \\
\text { NiII }\end{array}$ & $\begin{array}{l}3944.01 \\
3944.10 \\
3944.44 \\
3945.12 \\
3945.21 \\
3945.49\end{array}$ & 0.89 & -6.21 & $\begin{array}{l}0.79: \\
0.84:\end{array}$ & 20.4: & $\begin{array}{l}0.72 \\
0.53\end{array}$ & $-13.3:$ \\
\hline $\begin{array}{l}\text { FeI } \\
\mathrm{OI} \\
\mathrm{OI}(3) \\
\mathrm{FeI}(361) \\
\mathrm{OI} \\
\mathrm{FeI}\end{array}$ & $\begin{array}{l}3946.99 \\
3947.29 \\
3947.48 \\
3947.53 \\
3947.58 \\
3948.10\end{array}$ & 0.94 & -9.47 & 0.88: & & 0.74 & $-17.3:$ \\
\hline $\begin{array}{l}\mathrm{FeI}(604) \\
\text { LaII }\end{array}$ & $\begin{array}{l}3948.77 \\
3949.10\end{array}$ & 0.98 & -0.87 & & & $0.9:$ & $-10.6:$ \\
\hline $\begin{array}{l}\mathrm{FeI}(72) \\
\text { YII }\end{array}$ & $\begin{array}{l}3949.95 \\
3950.35\end{array}$ & $\begin{array}{l}0.99 \\
0.99\end{array}$ & $\begin{array}{c}-7.84 \\
-10.76\end{array}$ & & & 0.88 & -10.2 \\
\hline $\begin{array}{l}\text { FeI } \\
\text { VII(10) } \\
\text { FeI } \\
\text { FeI } \\
\text { FeI }\end{array}$ & $\begin{array}{l}3951.16 \\
3951.96 \\
3952.60 \\
3952.70 \\
3953.15\end{array}$ & $\begin{array}{l}0.99 \\
0.88\end{array}$ & $\begin{array}{l}-3.08 \\
-5.14\end{array}$ & & & 0.6 & -8.3 \\
\hline
\end{tabular}




\begin{tabular}{|c|c|c|c|c|c|c|c|}
\hline \multirow[t]{2}{*}{ Ident } & \multirow[t]{2}{*}{$\bar{\lambda}$} & \multicolumn{2}{|c|}{$\alpha$ Cyg } & \multicolumn{2}{|r|}{3 Pup } & \multicolumn{2}{|c|}{ IRAS 05040} \\
\hline & & $r$ & $V_{r}(\mathrm{~km} / \mathrm{s})$ & $r$ & $V_{r}(\mathrm{~km} / \mathrm{s})$ & $r$ & $\overline{V_{r}(\mathrm{~km} / \mathrm{s})}$ \\
\hline SiII & 3954.30 & & & & & & \\
\hline SiII & 3954.50 & & & & & & \\
\hline NiI & 3954.53 & 0.97 & -10.59 & & & & \\
\hline $\mathrm{FeI}(562)$ & 3955.34 & & & & & $0.92:$ & $-10:$ \\
\hline$\overline{\mathrm{FeI}}$ & 3956.46 & & & & & & \\
\hline $\mathrm{FeI}(278)$ & 3956.68 & 0.97 & -11.4 & $0.84:$ & & 0.79: & \\
\hline FeI & 3957.02 & & & & & & \\
\hline$\overline{\mathrm{ZrII}}$ & 3958.22 & 0.98 & -9.55 & & & $0.9:$ & $-8.3:$ \\
\hline FeII & 3959.18 & 0.99 & -3.94 & & & 0.93 & \\
\hline$\overline{\mathrm{FeII}}(212)$ & 3960.90 & 0.92 & -5.9 & & & 0.81: & -11: \\
\hline AlI(1) & 3961.52 & 0.86 & -7.13 & 0.73: & 21: & 0.65 & -12 \\
\hline $\mathrm{FeI}(562)$ & 3963.10 & 0.99 & -6.8 & & & 0.92: & $-10.2:$ \\
\hline $\mathrm{FeI}(361)$ & 3964.52 & & & & & & \\
\hline CrII(10) & 3964.64 & & & & & 0.7 & -15 \\
\hline $\mathrm{HeI}$ & 3964.73 & 0.9 & -11.25 & & & & \\
\hline FeI & 3966.06 & & & & & & \\
\hline FeII & 3966.13 & & & & & & \\
\hline FeII(3) & 3966.43 & & & & & 0.7 & -7.7 \\
\hline FeI & 3966.50 & & & & & & \\
\hline FeI & 3966.63 & & & & & & \\
\hline FeI & 3967.42 & & & & & & \\
\hline $\mathrm{FeI}$ & 3967.96 & & & & & & \\
\hline VII & 3968.11 & & & & & & \\
\hline CaII(1) & 3968.47 & 0.11 & -10.03 & & 23 & & -39.5 \\
\hline FeI & 3969.26 & & & & & & \\
\hline FeII & 3969.37 & & & & & & \\
\hline FeII & 3969.40 & & & & & & \\
\hline CrI & 3969.74 & & & & & & \\
\hline $\mathrm{HI}$ & 3970.07 & 0.21 & -6.33 & 0.13 & 33.9 & & -18.2 \\
\hline NiI & 3970.48 & & & & & & \\
\hline FeI & 3971.32 & & & & & & \\
\hline VII & 3973.64 & 0.9 & -2.27 & & & & \\
\hline FeII(29) & 3974.17 & 0.78 & -7.46 & & & 0.48 & -11.1 \\
\hline NiI & 3974.63 & & & & & & \\
\hline FeII(191) & 3975.02 & 0.92 & -8.77 & & & $0.8:$ & \\
\hline VII & 3977.73 & 0.96 & -4.08 & & & 0.81 & -12.1 \\
\hline FeI & 3977.74 & & & & & & \\
\hline$\overline{\mathrm{CrII}}(183)$ & 3979.51 & 0.85 & -4.65 & 0.83 & 23.6 & 0.62 & -10.5 \\
\hline FeII(3) & 3981.61 & & & $0.84:$ & & & \\
\hline $\mathrm{FeI}(278)$ & 3981.77 & & & & & & \\
\hline $\operatorname{TiII}(11)$ & 3981.99 & 0.92 & -13.67 & 0.87: & & 0.73: & \\
\hline FeII & 3982.27 & & & & & & \\
\hline YII(6) & 3982.59 & & & $0.87:$ & & & \\
\hline CoII & 3983.02 & & & & & & \\
\hline $\operatorname{CrI}(38)$ & \begin{tabular}{|c|}
3983.93 \\
\end{tabular} & 0.99 & -3.29 & 0.87: & 19: & $0.9:$ & $-14:$ \\
\hline CrII(10) & 3986.03 & & & & & & \\
\hline $\mathrm{FeI}(665)$ & 3986.17 & & & 0.89: & 21: & 0.92: & $-11:$ \\
\hline $\operatorname{MgI}(17)$ & 3986.75 & & & 0.87 & 20 & 0.85 & $-12:$ \\
\hline $\operatorname{MgI}(33)$ & 3987.08 & & & 0.89: & 17: & & \\
\hline TiII(11) & 3987.60 & 0.97 & -3.28 & 0.89 & 20.1 & $0.83:$ & $-11.2:$ \\
\hline 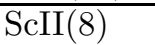 & 3989.06 & & & 0.92 & 16: & & \\
\hline$\overline{\operatorname{TiI}(12)}$ & 3989.76 & & & 0.91 & 22.8: & 0.91: & $-9:$ \\
\hline ZrII(30) & 3991.13 & 0.97 & -7.81 & & & 0.94: & $-7:$ \\
\hline NII & 3995.00 & 0.98 & -5.99 & & & & \\
\hline $\mathrm{CoI}$ & 3995.30 & & & & & & \\
\hline$\overline{\text { VII(9) }}$ & 3997.12 & 0.95 & -2.59 & & & 0.76 & $-9.5:$ \\
\hline
\end{tabular}




\begin{tabular}{|c|c|c|c|c|c|c|c|}
\hline \multirow[t]{2}{*}{ Ident } & \multirow[t]{2}{*}{$\lambda$} & \multicolumn{2}{|c|}{$\alpha \mathrm{Cyg}$} & \multicolumn{2}{|c|}{$3 \mathrm{Pup}$} & \multicolumn{2}{|c|}{ IRAS 05040} \\
\hline & & $r$ & $V_{r}(\mathrm{~km} / \mathrm{s})$ & $r$ & $V_{r}(\mathrm{~km} / \mathrm{s})$ & $r$ & $V_{r}(\mathrm{~km} / \mathrm{s})$ \\
\hline $\mathrm{FeI}(278)$ & 3997.39 & & & & & & \\
\hline $\operatorname{CoI}(32)$ & 3997.91 & & & & & & \\
\hline $\operatorname{FeI}(276)$ & 3998.06 & & & & & 0.91: & \\
\hline TiI(12) & 3998.64 & & & & & & \\
\hline ZrII(16) & 3998.95 & 0.98 & -4.49 & & & 0.94 & \\
\hline FeII $(29)$ & 4002.08 & 0.84 & & & & 0.59 & -9.3 \\
\hline CrII & 4002.48 & 0.85 & & & & & \\
\hline FeII(190) & 4002.54 & & & & & & \\
\hline SiII & 4002.59 & & & & & & \\
\hline $\operatorname{VII}(9)$ & 4002.94 & 0.93 & & & & 0.76 : & $-11.5:$ \\
\hline CrII(194) & 4003.28 & & & & & & \\
\hline FeII(127) & 4004.15 & 0.99 & & & & $0.95:$ & \\
\hline $\mathrm{FeI}(43)$ & 4005.24 & & & & & $0.7:$ & $-11.8:$ \\
\hline VII $(32)$ & 4005.71 & 0.86 & -8.41 & 0.8 & & 0.6 & -10.4 \\
\hline $\operatorname{HeI}(55)$ & 4009.26 & 0.96 & -4.12 & & & 0.94: & $-10.1:$ \\
\hline $\mathrm{FeI}(72)$ & 4009.71 & & & & & 0.9 & $-7.5:$ \\
\hline $\operatorname{TiII}(11)$ & 4012.38 & 0.74 & -1.93 & 0.74 & 24.2 & & -5.3 \\
\hline $\operatorname{FeII}(126)$ & 4012.46 & & & & & 0.42 & -11.1 \\
\hline CrII & 4012.50 & & & & & & \\
\hline $\operatorname{ScII}(8)$ & 4014.53 & & & & & 0.88 & -12 \\
\hline $\operatorname{NiII}(12)$ & 4015.47 & 0.87 & -4.95 & 0.87: & 24.7: & 0.68 & -10.8 \\
\hline $\mathrm{FeI}(278)$ & 4021.87 & & & & & 0.91: & $-10:$ \\
\hline $\mathrm{VII}(32)$ & 4023.38 & 0.9 & -6.61 & & & 0.66 & -8.8 \\
\hline ZrII & 4024.42 & & & & & & \\
\hline FeII(127) & 4024.55 & 0.79 & -3.6 & & & 0.52 & -9.2 \\
\hline $\mathrm{FeI}$ & 4024.73 & & & & & & \\
\hline TiII(11) & 4025.13 & 0.9 & & & & 0.6 & -10 \\
\hline $\mathrm{HeI}(18)$ & 4026.19 & 0.9 & -2.11 & & & $0.82:$ & $-10.5:$ \\
\hline $\mathrm{HeI}$ & 4026.20 & & & & & & \\
\hline $\mathrm{HeI}$ & 4026.36 & & & & & & \\
\hline $\operatorname{TiII}(87)$ & 4028.34 & 0.8 & -5.55 & \begin{tabular}{|l|}
0.82 \\
\end{tabular} & 31.1: & 0.48 & -8.9 \\
\hline SiII & 4028.47 & & & & & & \\
\hline CrII & 4030.36 & & & & & & \\
\hline $\mathrm{FeI}(560)$ & 4030.49 & 0.97 & -7.66 & & & & \\
\hline $\operatorname{MnI}(2)$ & 4030.75 & 0.98 & -5.94 & 0.84: & 18.5: & & \\
\hline TiII & 4030.82 & & & & & & \\
\hline FeII(151) & 4031.44 & 0.96 & -6.23 & & & 0.88 & $-11.1:$ \\
\hline FeII(126) & 4032.94 & 0.86 & -5.08 & $0.8:$ & 29.1: & 0.65 & $-11.5:$ \\
\hline $\operatorname{MnI}(2)$ & 4033.06 & & & & & & \\
\hline $\operatorname{MnI}(2)$ & 4034.48 & & & 0.87 & 17.8 & 0.9 & $-12:$ \\
\hline SiII & 4035.28 & & & & & & \\
\hline $\operatorname{VII}(32)$ & 4035.62 & 0.9 & -6.51 & 0.84 & 27.1 & 0.66 & -10.4 \\
\hline $\mathrm{MnI}$ & 4035.72 & & & & & & \\
\hline VII $(9)$ & 4036.78 & 0.98 & -7.32 & & & 0.87 & $-10.7:$ \\
\hline CrII(194) & 4037.97 & 0.93 & -4.85 & & & 0.8 & -11.7 \\
\hline $\mathrm{VII}(32)$ & 4039.56 & & & & & 0.96: & $-10:$ \\
\hline $\operatorname{MnI}(5)$ & 4041.36 & & & & & & \\
\hline $\mathrm{FeII}(172)$ & 4041.65 & & & & & 0.93 & \\
\hline $\mathrm{FeI}(2176)$ & 4043.90 & & & & & & \\
\hline $\operatorname{FeII}(172)$ & 4044.01 & 0.92 & -6.39 & & & $0.83:$ & $-12.1:$ \\
\hline $\mathrm{FeI}$ & 4045.59 & & & & & & \\
\hline ZrII & 4045.61 & & & & & & \\
\hline $\operatorname{FeI}(43)$ & 4045.81 & 0.78 & -6.09 & 0.75 & 29.6: & 0.46 & -10.3 \\
\hline ZrII & 4048.67 & & & & & & \\
\hline $\operatorname{MnI}(5)$ & 4048.74 & & & & & & \\
\hline
\end{tabular}




\begin{tabular}{|c|c|c|c|c|c|c|c|}
\hline \multirow[t]{2}{*}{ Ident } & \multirow[t]{2}{*}{$\bar{\lambda}$} & \multicolumn{2}{|c|}{$\alpha$ Cyg } & \multicolumn{2}{|r|}{3 Pup } & \multicolumn{2}{|c|}{ IRAS 05040} \\
\hline & & $r$ & $V_{r}(\mathrm{~km} / \mathrm{s})$ & $r$ & $V_{r}(\mathrm{~km} / \mathrm{s})$ & $r$ & $\overline{V_{r}(\mathrm{~km} / \mathrm{s})}$ \\
\hline $\begin{array}{l}\text { FeII(172) } \\
\text { CrII(193) }\end{array}$ & $\begin{array}{l}4048.83 \\
4049.10\end{array}$ & 0.86 & -2.52 & & & 0.7 & -8.4 \\
\hline VII & 4051.05 & & & & & & \\
\hline $\mathrm{FeII}(172)$ & 4051.21 & & & & & 0.88 & -11.1: \\
\hline VII & 4051.29 & & & & & & \\
\hline CrII(19) & 4051.93 & 0.87 & -5.33 & & & 0.62 & -7.4 \\
\hline CrII & 4053.43 & & & & & & \\
\hline VII & 4053.59 & & & & & & \\
\hline TiII(87) & 4053.82 & 0.81 & -0.65 & 0.8 & 34.4: & 0.5 & $-8:$ \\
\hline CrII(19) & 4054.08 & & & & & & \\
\hline CrII & 4056.06 & & & & & & \\
\hline TiII(11) & 4056.19 & 0.98 & -7.6 & & & 0.91 & -9.9 : \\
\hline$\overline{F e I I}(212)$ & 4057.46 & 0.93 & $\begin{array}{l}-6.39 \\
\end{array}$ & $0.86:$ & 21.3: & $0.8:$ & $-12:$ \\
\hline $\operatorname{MgI}(16)$ & 4057.51 & & & & & & \\
\hline FeII(18() & 4061.78 & 0.97 & -6.13 & & & $0.92:$ & $-11.4:$ \\
\hline$\overline{\mathrm{FeI}(359)}$ & 4062.44 & & & & & 0.97: & $-7.4:$ \\
\hline FeI & 4063.28 & & & & & & \\
\hline $\mathrm{FeI}(43)$ & 4063.59 & 0.85 & -6.37 & 0.77 & 22.3 & 0.51 & -10.7 \\
\hline $\operatorname{TiII}(106)$ & 4064.35 & & & & & 0.94: & \\
\hline VII(215) & 4065.07 & & & & & $0.95:$ & $-8:$ \\
\hline FeI(398) & 4066.97 & & & & & & \\
\hline $\operatorname{NiII}(11)$ & 4067.03 & 0.73 & -4.85 & 0.82 & 25.8 & 0.55 & $-9:$ \\
\hline FeII(188) & 4069.88 & & & & & & \\
\hline FeII $(22)$ & 4070.03 & 0.94 & -8.96 & & & 0.96 : & -16.8: \\
\hline $\mathrm{FeI}(558)$ & 4070.77 & & & & & & \\
\hline CrII(193) & 4070.84 & 0.94 & -3.79 & & & 0.82 & $-7.3:$ \\
\hline FeI(43) & 4071.74 & 0.87 & -6.58 & 0.8 & 21.7 & 0.58 & -11.7 \\
\hline $\operatorname{CrII}(26)$ & 4072.56 & 0.95 & -5.51 & & & 0.8 & -11 \\
\hline SiII & 4072.71 & & & & & & \\
\hline SiII & 4075.45 & 0.94 & -4.43 & & & $0.88:$ & $-12.1:$ \\
\hline CrII & 4075.62 & & & & & & \\
\hline FeII $(21)$ & 4075.95 & & & & & $0.88:$ & $-13.2:$ \\
\hline SiII & 4076.78 & 0.92 & -4.06 & & & 0.8 & -10 \\
\hline CrII & 4076.89 & & & & & & \\
\hline CrII & 4077.51 & & & & & & \\
\hline $\operatorname{SrII}(1)$ & 4077.71 & 0.82 & -7.49 & 0.78 & 20.6 & 0.5 & -11.6 \\
\hline CrII(165) & 4082.29 & 0.96 & -4.68 & & & $0.88:$ & $-11.2:$ \\
\hline $\operatorname{MnI}(5)$ & 4083.63 & & & & & 0.91 & -6.1 : \\
\hline $\mathrm{FeI}(697)$ & 4083.77 & & & & & & \\
\hline FeI(698) & 4084.49 & & & & & $0.93:$ & $-11.4:$ \\
\hline FeI(559) & 4085.30 & & & & & $0.92:$ & $-8.5:$ \\
\hline CrII(26) & 4086.13 & 0.96 & -5.6 & & & 0.83 & -11.3 \\
\hline$\overline{\mathrm{FeII}}(28)$ & 4087.28 & 0.96 & $\begin{array}{l}-1.99 \\
\end{array}$ & & & 0.84 & -11.7 \\
\hline CrII(19) & 4087.59 & & & & & & \\
\hline FeII(39) & 4088.76 & 0.97 & -3.01 & & & $0.89:$ & $-9.8:$ \\
\hline CrII & 4088.83 & & & & & & \\
\hline FeII & 4093.23 & 0.98 & -5.28 & & & $0.9:$ & \\
\hline FeII & 4097.50 & 0.94 & -3.47 & & & & \\
\hline FeI & 4098.18 & & & & & & \\
\hline CrII(165) & 4098.41 & 0.9 & -4.12 & & & & $-11.4:$ \\
\hline NI & 4099.94 & & & & & & \\
\hline HI & 4101.73 & 0.23 & -5.56 & 0.12 & 26.1 & 0.04 & -16.3 \\
\hline SiI & 4102.94 & & & & & & \\
\hline MnII & 4104.98 & & & & & & \\
\hline $\mathrm{FeI}(354)$ & 4107.49 & & & & & & -13.9: \\
\hline$\overline{\mathrm{FeI}}(375)$ & 4109.80 & & & & & 0.86 & -10.9 \\
\hline
\end{tabular}




\begin{tabular}{|c|c|c|c|c|c|c|c|}
\hline \multirow[t]{2}{*}{ Ident } & \multirow[t]{2}{*}{$\lambda$} & \multicolumn{2}{|c|}{$\alpha \mathrm{Cyg}$} & \multicolumn{2}{|c|}{$3 \mathrm{Pup}$} & \multicolumn{2}{|c|}{ IRAS 05040} \\
\hline & & $r$ & $V_{r}(\mathrm{~km} / \mathrm{s})$ & $r$ & $V_{r}(\mathrm{~km} / \mathrm{s})$ & $r$ & $\overline{V_{r}(\mathrm{~km} / \mathrm{s})}$ \\
\hline $\mathrm{NI}$ & 4109.95 & 0.95 & -10.47 & & & & \\
\hline MnII & 4110.62 & & & & & & \\
\hline CrII(18) & 4110.99 & 0.87 & -6 & & 18.5: & 0.63 & -10.2 \\
\hline CrII & 4111.00 & & & & & & \\
\hline FeII(188) & 4111.88 & 0.96 & -4.15 & & & 0.87 & -14.9 \\
\hline CrII(18) & 4112.54 & & & & & 0.93: & $-5.6:$ \\
\hline $\operatorname{CrII}(18)$ & 4113.21 & 0.96 & -7.33 & & & 0.84 & -10.7 \\
\hline $\mathrm{FeI}(375)$ & 4114.45 & & & & & 0.98 & \\
\hline $\mathrm{FeI}(700)$ & 4117.85 & & & & & $0.96:$ & $-10:$ \\
\hline $\operatorname{FeI}(801)$ & 4118.55 & 0.97 & -7.09 & & & 0.86 & -11.7 \\
\hline $\mathrm{FeII}(21)$ & 4119.52 & 0.94 & -5.94 & & & 0.81 & -10.8 \\
\hline $\mathrm{HeI}$ & 4120.81 & & & & & & \\
\hline $\operatorname{HeI}(16)$ & 4120.82 & 0.97 & -1.07 & & & 0.95: & $-11.8:$ \\
\hline FeII $(28)$ & 4122.67 & 0.72 & -5.97 & 0.82 & 23.6 & 0.43 & -10 \\
\hline $\operatorname{FeII}(22)$ & 4124.79 & 0.91 & -5.51 & 0.9: & 25: & 0.71 & -12.6 \\
\hline $\mathrm{FeI}(695)$ & & & & & & 0.96 & \\
\hline CrII(181) & 4127.06 & & & & & 0.94: & $-12.3:$ \\
\hline $\mathrm{FeI}$ & 4127.61 & & & & & & \\
\hline $\operatorname{SiII}(3)$ & 4128.05 & 0.6 & -3.98 & 0.79 & 28.1: & 0.42 & -7.7 \\
\hline $\operatorname{FeII}(27)$ & 4128.75 & 0.78 & -9.39 & & 23.4 & 0.52 & -11.1 \\
\hline TiII & 4129.16 & & & & & & \\
\hline SiII & 4130.87 & & & & & & \\
\hline $\operatorname{SiII}(3)$ & 4130.89 & 0.6 & -5.85 & 0.77 & 30.7 & 0.47 & -13.2 \\
\hline FeI (43) & 4132.06 & 0.93 & 5.77 & $0.84:$ & 20.1: & 0.7 & -10.4 \\
\hline $\operatorname{CrII}(26)$ & 4132.42 & & & & & & \\
\hline $\operatorname{FeI}(357)$ & 4134.68 & 0.99 & -6.93 & & & 0.9 & -12.3 \\
\hline MnII & 4136.90 & 0.97 & -2.91 & & & 0.88: & $-8.3:$ \\
\hline $\operatorname{FeI}(726)$ & 4137.00 & & & & & & \\
\hline FeII(150) & 4138.21 & & & & & & $-2.9:$ \\
\hline $\operatorname{FeII}(39)$ & 4138.41 & 0.93 & -11.28 & & & $0.77:$ & $-16.3:$ \\
\hline FeII & 4139.72 & 0.99 & -4.14 & & & $0.96:$ & $-15.2:$ \\
\hline $\operatorname{SII}(44)$ & 4142.26 & 0.99 & -6.22 & & & $0.96:$ & \\
\hline $\mathrm{FeI}(523)$ & 4143.41 & & & & & 0.8 & $-11.5:$ \\
\hline NI & 4143.43 & & & & & & \\
\hline $\operatorname{HeI}(53)$ & 4143.76 & & & & & & \\
\hline $\mathrm{FeI}(43)$ & 4143.87 & 0.9 & -10.4 & 0.86 & 21.3: & 0.59 & -13.2 \\
\hline $\mathrm{SII}(44)$ & 4145.06 & & & & & 0.9 & $-8.3:$ \\
\hline CoII & 4145.14 & 0.97 & -5.73 & & & & \\
\hline $\operatorname{CrII}(162)$ & 4145.78 & 0.88 & -5.76 & 0.91: & 31.5: & 0.7 & -12.6 \\
\hline CrII & 4146.39 & & & & & & \\
\hline FeII(141) & 4147.27 & 0.98 & -5.85 & & & 0.95: & $-13.4:$ \\
\hline $\mathrm{FeI}(42)$ & 4147.67 & & & & & 0.96: & $-12:$ \\
\hline $\operatorname{ZrII}(41)$ & 4149.20 & 0.96 & -3.77 & & & 0.91: & $-8.9:$ \\
\hline $\mathrm{FeI}(694)$ & 4149.37 & & & & & & \\
\hline $\operatorname{ZrII}(10)$ & 4150.99 & & & & & 0.96: & \\
\hline CrII & 4150.99 & & & & & & \\
\hline NI & 4151.48 & 0.97 & -7.13 & & & & \\
\hline FeII & 4153.01 & 0.98 & -3.49 & & & 0.95 & -10.2 \\
\hline $\operatorname{SII}(44)$ & 4153.07 & & & & & & \\
\hline $\mathrm{FeI}(695)$ & 4153.90 & & & & & 0.92 & $-10.9:$ \\
\hline $\operatorname{ZrII}(29)$ & 4156.23 & & & & & 0.96: & $-7.2:$ \\
\hline $\operatorname{FeI}(354)$ & 4156.80 & & & & & 0.93: & 13: \\
\hline $\mathrm{FeI}(695)$ & 4157.78 & & & & & 0.95: & $-10.5:$ \\
\hline FeII(12) & 4158.45 & & & & & $0.95:$ & -11.6 \\
\hline FeII(39) & 4160.62 & & & & & & \\
\hline
\end{tabular}




\begin{tabular}{|c|c|c|c|c|c|c|c|}
\hline \multirow[t]{2}{*}{ Ident } & \multirow[t]{2}{*}{$\bar{\lambda}$} & \multicolumn{2}{|c|}{$\alpha$ Cyg } & \multicolumn{2}{|c|}{3 Pup } & \multicolumn{2}{|c|}{ IRAS 05040} \\
\hline & & $r$ & $V_{r}(\mathrm{~km} / \mathrm{s})$ & $r$ & $V_{r}(\mathrm{~km} / \mathrm{s})$ & $r$ & $V_{r}(\mathrm{~km} / \mathrm{s})$ \\
\hline CoII & 4160.66 & 0.95 & -3.52 & & & 0.82 & -6 \\
\hline CrII & 4161.08 & & & & & & \\
\hline ZrII(42) & 4161.20 & & & & & 0.89 & \\
\hline $\operatorname{TiII}(21)$ & 4161.53 & 0.93 & -8.44 & 0.88 & 20.3 & 0.75 & $-9.6:$ \\
\hline $\begin{array}{c}\operatorname{SII}(44) \\
\end{array}$ & 4162.67 & 0.98 & -6.67 & & & $0.93:$ & \\
\hline TiII(105) & 4163.64 & 0.75 & -5.63 & 0.79 & 33.5: & 0.5 & -7.8 \\
\hline $\operatorname{MgI}(15)$ & 4167.27 & 0.97 & -7.4 & 0.91 & 20: & $0.85:$ & $-13.5:$ \\
\hline FeII $(22)$ & 4168.66 & & & & & 0.93 & $-10:$ \\
\hline CrII $(86)$ & 4170.86 & & & & & \begin{tabular}{|l|}
$0.9:$ \\
\end{tabular} & $-15:$ \\
\hline CrII & 4171.90 & & & & & & \\
\hline TiII(105) & 4171.90 & 0.78 & -5.04 & 0.79 & 38.5: & 0.5 & -7.9 \\
\hline FeI & 4172.12 & & & & & & \\
\hline CrII(18) & 4172.59 & & & & & 0.9: & $-8.9:$ \\
\hline FeII(27) & 4173.46 & 0.52 & -4.56 & 0.66 & 34.5 & 0.8 & -4.5 \\
\hline TiII(21) & 4173.53 & & & & & & -9.8 \\
\hline TiII(105) & 4174.07 & & & & & 0.79: & \\
\hline SII(65) & 4174.27 & & & & & & -14 \\
\hline MnII & 4174.32 & & & & & & \\
\hline FeI $(354)$ & 4175.64 & 0.99 & -3.92 & & & $0.94:$ & $-8:$ \\
\hline FeI(689) & 4176.57 & & & & & $0.94:$ & $-12.3:$ \\
\hline YII & 4177.53 & & & & & & \\
\hline FeII $(21)$ & 4177.69 & 0.72 & -5.61 & 0.81 & 27.1: & 0.49 & -9.1 \\
\hline $\operatorname{VII}(25)$ & 4178.40 & & & & & 0.79 & \\
\hline PII & 4178.46 & & & & & & \\
\hline FeII(28) & 4178.86 & 0.5 & -4.5 & 0.86 & 27.2 & 0.31 & -3.4 \\
\hline CrII(26) & 4179.42 & & & & & 0.63: & -11.1: \\
\hline ZrII & 4179.81 & & & & & & \\
\hline $\begin{array}{l}\mathrm{VII}(19) \\
\end{array}$ & 4180.86 & & & & & \begin{tabular}{|l|}
0.95 \\
\end{tabular} & -11.5 \\
\hline FeII(148) & 4180.98 & 0.99 & -7.73 & & & & \\
\hline$\overline{\mathrm{FeI}(354)}$ & 4181.75 & 0.98 & -5.86 & $0.94:$ & 21.4: & 0.84 & $-11.2:$ \\
\hline FeII $(21)$ & 4183.20 & & & & & & \\
\hline VII(37) & 4183.43 & 0.97 & -7.35 & & & 0.83 & -11.4: \\
\hline TiII(21) & 4184.30 & 0.96 & -4.61 & & & 0.85 & -10.3 \\
\hline FeI $(355)$ & 4184.89 & & & & & & \\
\hline ZrII & 4186.67 & & & & & & \\
\hline FeI(152) & 4187.04 & 0.97 & -2.97 & 0.91 & 20.6 & 0.84 & -12.7 \\
\hline SiII & 4187.13 & & & & & & \\
\hline SiII & 4187.15 & & & & & & \\
\hline $\mathrm{FeI}(152)$ & 4187.80 & & & 0.87 & 20.7 & 0.7 & -9.9 \\
\hline NiII & 4187.85 & 0.9 & -7.22 & & & & \\
\hline $\mathrm{FeI}(1116)$ & 4188.73 & & & & & 0.95 & $-12.4:$ \\
\hline TiII(21) & 4190.23 & & & & & 0.94: & \\
\hline SiII & 4190.71 & & & & & & \\
\hline $\mathrm{FeI}(152)$ & 4191.43 & & & & & 0.89: & $-11.2:$ \\
\hline $\operatorname{NiII}(10)$ & 4192.07 & 0.95 & -6.67 & & & 0.85 & $-13.7:$ \\
\hline $\operatorname{MgII}(28)$ & 4193.49 & 0.99 & -7.11 & & & $0.96:$ & $-15.8:$ \\
\hline FeI(693) & 4195.33 & & & & & & \\
\hline CrII(161) & 4195.42 & 0.95 & -6.35 & & & 0.83 & $-12.5:$ \\
\hline FeI(693) & 4196.21 & & & & & 0.92: & $-12.9:$ \\
\hline SiII & 4198.13 & & & & & & \\
\hline FeI & 4198.25 & & & & & & \\
\hline $\mathrm{FeI}(152)$ & 4198.30 & 0.97 & -8.79 & $0.87:$ & 18.4: & 0.82 & -13.6 \\
\hline FeI & 4198.63 & & & & & & \\
\hline FeI(522) & 4199.10 & 0.95 & -3.14 & $0.9:$ & 20.5: & 0.8 & -10.4 \\
\hline MnII & 4200.27 & & & & & & \\
\hline FII & 4200.52 & & & & & & \\
\hline
\end{tabular}




\begin{tabular}{|c|c|c|c|c|c|c|c|}
\hline \multirow[t]{2}{*}{ Ident } & \multirow[t]{2}{*}{$\lambda$} & \multicolumn{2}{|c|}{$\alpha \mathrm{Cyg}$} & \multicolumn{2}{|c|}{$3 \mathrm{Pup}$} & \multicolumn{2}{|c|}{ IRAS 05040} \\
\hline & & $r$ & $\overline{V_{r}(\mathrm{~km} / \mathrm{s})}$ & $r$ & $V_{r}(\mathrm{~km} / \mathrm{s})$ & $r$ & $V_{r}(\mathrm{~km} / \mathrm{s})$ \\
\hline SiII & 4200.66 & & & & & & \\
\hline SiII & 4200.90 & & & & & & \\
\hline $\mathrm{FeI}$ & 4200.94 & & & & & & \\
\hline $\mathrm{FeI}(42)$ & 4202.03 & & & 0.9 & 25: & 0.7 & -9 \\
\hline $\mathrm{VII}(25)$ & 4202.36 & & & & & $0.77:$ & $-13:$ \\
\hline FeII & 4202.52 & & & & & & \\
\hline FeII & 4202.86 & & & & & & \\
\hline $\mathrm{FeI}(355)$ & 4203.98 & 0.99 & -3.09 & 0.93 & 18.6 & $0.93:$ & $-11.3:$ \\
\hline YII & 4204.70 & & & & & & \\
\hline VII & 4205.04 & & & & & & \\
\hline $\mathrm{VII}(37)$ & 4205.08 & & & & & & \\
\hline $\operatorname{MnII}(2)$ & 4205.38 & 0.93 & -12.33 & 0.91: & 26.3: & $0.75:$ & \\
\hline $\mathrm{FeII}(22)$ & 4205.60 & & & & & & \\
\hline $\operatorname{MnII}(7)$ & 4206.37 & 0.97 & -5.52 & & & $0.87:$ & $-11.1:$ \\
\hline MnII & 4207.23 & & & & & & \\
\hline $\operatorname{CrII}(26)$ & 4207.36 & 0.97 & -5.07 & & & $0.85:$ & -12.9 : \\
\hline $\mathrm{FeI}(689)$ & 4208.61 & & & & & $0.96:$ & \\
\hline $\mathrm{ZrII}(41)$ & 4208.98 & 0.98 & -4.77 & & & 0.93 & $-9.5:$ \\
\hline $\operatorname{CrII}(180)$ & 4209.09 & & & & & 0.94 & \\
\hline VII & 4209.76 & & & & & & \\
\hline $\mathrm{FeI}(152)$ & 4210.34 & 0.99 & -4.73 & & & 0.91 & -11.4 \\
\hline $\mathrm{ZrII}(15)$ & 4211.88 & 0.99 & -7.07 & & & $0.93:$ & $-14:$ \\
\hline FeII & 4213.52 & 0.99 & -5.2 & & & $0.95:$ & $-12.9:$ \\
\hline $\operatorname{FeI}(355)$ & 4213.65 & & & & & & \\
\hline NI & 4214.80 & & & & & & \\
\hline $\operatorname{SrII}(1)$ & 4215.52 & 0.88 & -2.84 & 0.85 & 36.6: & 0.61 & -10 \\
\hline CrII(18) & 4215.74 & & & & & & \\
\hline NI & 4216.09 & & & & & & \\
\hline $\operatorname{CrII}(18)$ & 4217.06 & 0.98 & -1.81 & 0.96 & 32.2 & 0.94 & $-10.3:$ \\
\hline $\operatorname{TiII}(96)$ & 4217.34 & & & & & & \\
\hline $\mathrm{FeI}(693)$ & 4217.55 & & & 0.94 & 18.7: & & \\
\hline NI & 4218.86 & & & & & & \\
\hline $\operatorname{FeI}(800)$ & 4219.36 & 0.98 & -4.5 & 0.95 & 19.6 & 0.92 & $-13.3:$ \\
\hline $\mathrm{FeI}(482)$ & 4220.34 & & & $0.96:$ & 18.7: & & \\
\hline CrII & 4222.00 & & & & & & \\
\hline NI & 4222.10 & & & & & & \\
\hline $\mathrm{FeI}(152)$ & 4222.21 & 0.98 & -8.66 & 0.93 & 19.4 & 0.91 & -13.8 \\
\hline ZrII & 4222.38 & & & & & & \\
\hline NI & 4223.13 & 0.98 & -6.22 & & & $0.95:$ & \\
\hline $\mathrm{FeI}(689)$ & 4224.17 & & & & & $0.92:$ & $-13:$ \\
\hline $\mathrm{CrII}(162)$ & 4224.86 & 0.9 & -3.88 & 0.91 & 25.3: & 0.74 & $-11.5:$ \\
\hline NI & 4224.88 & & & & & & \\
\hline VII(37) & 4225.21 & & & 0.9 & 29.2: & & \\
\hline $\mathrm{FeI}$ & 4225.45 & & & & & & \\
\hline $\mathrm{CaI}(2)$ & 4226.73 & 0.96 & -7.94 & 0.9 & 30: & 0.73 & -11.3 \\
\hline FeII & 4227.17 & & & & & & \\
\hline TiII(33) & 4227.33 & 0.94 & -2.88 & 0.91: & 20.4: & 0.73 & -13.8 \\
\hline $\operatorname{FeI}(693)$ & 4227.43 & & & & & & \\
\hline $\mathrm{CI}(17)$ & 4228.30 & & & & & $0.95:$ & $-11:$ \\
\hline $\mathrm{NiI}(136)$ & 4231.02 & & & 0.97: & 18.8 & & \\
\hline SiII & 4232.85 & & & & & & \\
\hline $\mathrm{FeII}(27)$ & 4233.17 & 0.41 & -4.65 & 0.49 & 32.9: & 0.25 & -5.1 \\
\hline CrII & 4233.24 & & & & & & \\
\hline $\mathrm{FeI}(152)$ & 4233.60 & & & & & & \\
\hline YII & 4235.73 & & & & & & \\
\hline $\mathrm{FeI}(152)$ & 4235.94 & 0.96 & -3.74 & 0.91 & 30.2 : & 0.81 & -12.1 \\
\hline
\end{tabular}




\begin{tabular}{|c|c|c|c|c|c|c|c|}
\hline \multirow[t]{2}{*}{ Ident } & \multirow[t]{2}{*}{$\lambda$} & \multicolumn{2}{|c|}{$\alpha$ Cyg } & \multicolumn{2}{|r|}{ 3Pup } & \multicolumn{2}{|c|}{ IRAS 05040} \\
\hline & & $r$ & $V_{r}(\mathrm{~km} / \mathrm{s})$ & $r$ & $V_{r}(\mathrm{~km} / \mathrm{s}$ & $r$ & $V_{r}(\mathrm{~km} / \mathrm{s})$ \\
\hline CrII(117) & 4236.38 & & & & & & \\
\hline $\mathrm{FeI}(689)$ & 4238.03 & & & & & $0.94:$ & $-12:$ \\
\hline MnII & 4238.79 & 0.96 & -4.31 & & & & \\
\hline $\mathrm{FeI}(693)$ & 4238.81 & & & 0.93 & 19.9 & 0.8 & -11.8 \\
\hline MnII & 4239.19 & & & & & & \\
\hline MnII & 4242.33 & & & & & & \\
\hline CrII(31) & 4242.36 & 0.7 & -4.65 & 0.79 & 31.7: & 0.47 & -6.7 \\
\hline MgII & 4242.45 & & & & & & \\
\hline MgII & 4242.54 & & & & & & \\
\hline $\operatorname{MnII}(7)$ & 4244.25 & & & & & 0.94 & \\
\hline $\operatorname{NiII}(9)$ & 4244.78 & 0.96 & -6.85 & & & 0.88 & $-12.5:$ \\
\hline MnII & 4245.92 & & & & & & \\
\hline CrII(31) & 4246.41 & & & & & 0.93: & $-13:$ \\
\hline $\operatorname{ScII}(7)$ & 4246.82 & 0.78 & -4.68 & 0.77 & 37 & 0.53 & -3.5 \\
\hline $\mathrm{FeI}(693)$ & 4247.43 & & & & & 0.87 & \\
\hline $\mathrm{FeI}(152)$ & 4250.12 & & & 0.94: & 19: & \begin{tabular}{|l|}
0.8 \\
\end{tabular} & -8.7 \\
\hline FeII & 4250.44 & & & & & & \\
\hline $\mathrm{FeI}(42)$ & 4250.79 & 0.93 & -16.72 & 0.88 & 17.6: & 0.76 & -13.1 \\
\hline MnII & 4251.72 & 0.96 & -4.17 & & & 0.89: & $-9.9:$ \\
\hline CrII(31) & 4252.63 & 0.88 & -3.22 & 0.9: & 25.7 & 0.67 & -9.6 \\
\hline MnII & 4252.96 & & & & & & \\
\hline NI & 4253.40 & & & & & & \\
\hline $\mathrm{CrI}(1)$ & 4254.34 & 0.92 & 4.28 & 0.91 & $36.3:$ & 0.73 & -11.3 \\
\hline VII(18) & 4254.40 & & & & & & \\
\hline CrII & 4254.52 & & & & & & \\
\hline NI & 4254.73 & & & & & & \\
\hline NI & 4255.16 & & & & & & \\
\hline CrII(192) & 4256.11 & 0.99 & -7 & & & \begin{tabular}{|l|}
0.97 \\
\end{tabular} & \\
\hline ZrII & 4258.04 & & & & & & \\
\hline FeII(28) & 4258.15 & 0.77 & -3.07 & 0.82 & 35: & 0.53 & -7.1 \\
\hline FeII & 4258.34 & & & & & & \\
\hline NI & 4258.71 & & & & & & \\
\hline SiII & 4259.19 & & & & & & \\
\hline $\operatorname{MnII}(7)$ & 4259.20 & 0.97 & -5.25 & & & 0.89 & $-13:$ \\
\hline MnII & 4260.47 & & & & & & \\
\hline $\mathrm{FeI}(152)$ & 4260.47 & 0.92 & -5.22 & 0.88 & 29.7: & 0.69 & -11.7 \\
\hline NI & 4261.28 & & & & & & \\
\hline CrII & 4261.85 & & & & & & \\
\hline CrII(31) & 4261.91 & 0.76 & -5.2 & 0.82 & 30.5: & 0.51 & -7.6 \\
\hline $\operatorname{VII}(24)$ & 4263.84 & & & & & & -14 \\
\hline NI & 4263.22 & & & & & & \\
\hline FeII & 4263.87 & 0.96 & -5.99 & & & \begin{tabular}{|l|}
0.89 \\
\end{tabular} & -15.8 \\
\hline CII & 4267.00 & & & & & & \\
\hline CII $(6)$ & 4267.26 & 0.97 & -11.6 & & & 0.91 & -10.6 \\
\hline $\operatorname{SII}(49)$ & 4267.76 & 0.98 & -8.11 & & & 0.93: & $-10.6:$ \\
\hline CrII & 4268.88 & & & & & & \\
\hline CrII(31) & 4269.28 & 0.91 & -6.28 & 0.93 & 31.9: & 0.73 & -10.7 \\
\hline $\mathrm{FeI}(152)$ & 4271.15 & & & & & \begin{tabular}{|l|}
0.79 \\
\end{tabular} & $-12.4:$ \\
\hline $\mathrm{FeI}(42)$ & 4271.76 & 0.87 & -6.06 & 0.84 & 28: & 0.55 & -10.4 \\
\hline FeII(27) & 4273.33 & 0.75 & -5.28 & 0.82 & 35.4: & 0.53 & -8.7 \\
\hline $\operatorname{CrI}(1)$ & 4274.80 & & & 0.93 & 18.8: & \begin{tabular}{|l|l|}
0.83 \\
\end{tabular} & $-11.8:$ \\
\hline CrII(31) & 4275.57 & 0.81 & -5.76 & 0.86 & 28.5: & 0.59 & -8.9 \\
\hline ZrII & 4277.37 & & & & & & \\
\hline TiII & 4277.54 & & & & & & \\
\hline & 4278.13 & & & & & & \\
\hline FeII(32) & 4278.16 & 0.87 & -6.03 & 0.9 & 35.3: & 0.68 & -11 \\
\hline
\end{tabular}




\begin{tabular}{|c|c|c|c|c|c|c|c|}
\hline \multirow[t]{2}{*}{ Ident } & \multirow[t]{2}{*}{$\lambda$} & \multicolumn{2}{|c|}{$\alpha \mathrm{Cyg}$} & \multicolumn{2}{|c|}{$3 \mathrm{Pup}$} & \multicolumn{2}{|c|}{ IRAS 05040} \\
\hline & & $r$ & $V_{r}(\mathrm{~km} / \mathrm{s})$ & $r$ & $V_{r}(\mathrm{~km} / \mathrm{s})$ & $r$ & $V_{r}(\mathrm{~km} / \mathrm{s})$ \\
\hline NiII & 4279.22 & 0.98 & -7.87 & & & & \\
\hline $\begin{array}{l}\mathrm{FeI}(71) \\
\text { MnII }\end{array}$ & $\begin{array}{l}4282.40 \\
4282.49\end{array}$ & 0.96 & -5.01 & 0.93 & 20.1: & 0.84 & -10.5 \\
\hline $\begin{array}{l}\operatorname{MnII}(6) \\
\mathrm{CrII}(31) \\
\operatorname{MnII}\end{array}$ & $\begin{array}{l}4283.77 \\
4284.19 \\
4284.43\end{array}$ & 0.85 & -5.15 & 0.87 & 33.4: & $\begin{array}{c}0.89: \\
0.64\end{array}$ & $\begin{array}{l}-15: \\
-8.7\end{array}$ \\
\hline $\mathrm{FeI}(597)$ & 4285.45 & & & 0.96 & 18.2 & 0.93 & $-15:$ \\
\hline $\begin{array}{l}\text { VII }(26) \\
\text { FeII }\end{array}$ & $\begin{array}{l}4286.13 \\
4286.28\end{array}$ & 0.96 & -5.84 & & & 0.91 & $-11:$ \\
\hline$[\mathrm{FeII}] 7 \mathrm{~F}$ & 4287.40 & & & 1.05 & 6.8 & & \\
\hline $\operatorname{TiII}(20)$ & 4287.87 & 0.9 & -5.02 & 0.91 & 39.5: & 0.67 & -7.9 \\
\hline $\begin{array}{l}\text { CrI(1) } \\
\text { TiII(41) } \\
\text { TiII } \\
\end{array}$ & $\begin{array}{l}4289.72 \\
4290.21 \\
4290.35 \\
\end{array}$ & 0.3 & -5.44 & 0.74 & 32.3 & $\begin{array}{l}0.82 \\
0.43\end{array}$ & $\begin{array}{l}-11.6 \\
-4.7\end{array}$ \\
\hline $\operatorname{MnII}(6)$ & 4292.24 & 0.98 & -5.05 & & & 0.91 & -12.3 \\
\hline $\begin{array}{l}\text { NI } \\
\text { TiII(20) } \\
\text { FeI } \\
\text { SII } \\
\text { ScII(15) }\end{array}$ & $\begin{array}{l}4293.21 \\
4294.09 \\
4294.12 \\
4294.40 \\
4294.77\end{array}$ & 0.69 & -4.72 & 0.71 & 34.3: & $\begin{array}{c}0.4 \\
0.93:\end{array}$ & -9.8 : \\
\hline $\operatorname{FeII}(28)$ & 4296.57 & 0.64 & -4.86 & 0.76 & 34.1 & 0.44 & -6.2 \\
\hline $\begin{array}{l}\text { FeI(152) } \\
\text { TiII(41) }\end{array}$ & $\begin{array}{l}4299.23 \\
4300.04\end{array}$ & 0.59 & -5.04 & 0.85 & 32.4 & $\begin{array}{l}0.83: \\
0.34\end{array}$ & $\begin{array}{c}-13.1 \\
-3.8\end{array}$ \\
\hline $\begin{array}{l}\text { TiII }(41) \\
\mathrm{CaI} \\
\mathrm{FeII}(27)\end{array}$ & $\begin{array}{l}4301.91 \\
4302.53 \\
4303.18\end{array}$ & $\begin{array}{l}0.79 \\
0.53 \\
\end{array}$ & $\begin{array}{l}-4.36 \\
-4.62 \\
\end{array}$ & $\begin{array}{l}0.82 \\
0.67 \\
\end{array}$ & $\begin{array}{c}31 \\
33.7:\end{array}$ & $\begin{array}{l}0.52 \\
0.35 \\
\end{array}$ & $\begin{array}{c}-6 \\
-5.7\end{array}$ \\
\hline $\operatorname{ScII}(15)$ & 4305.71 & 0.98 & 10.96 & & & 0.94 & -13.2 : \\
\hline $\begin{array}{l}\text { CrII } \\
\text { TiII(41) } \\
\text { FeI } \\
\text { MnII }\end{array}$ & $\begin{array}{l}4306.92 \\
4307.86 \\
4307.90 \\
4308.16\end{array}$ & $\begin{array}{l}0.98 \\
0.73\end{array}$ & $\begin{array}{l}-1.56 \\
-4.59\end{array}$ & 0.77 & 28.7 & $\begin{array}{l}0.91 \\
0.47\end{array}$ & $\begin{array}{c}-12.3: \\
-4.4\end{array}$ \\
\hline $\begin{array}{l}\text { VII } \\
\text { TiII }(41) \\
\text { FeII } \\
\text { ScII }(15) \\
\text { FeII }(32) \\
\text { TiII }(41) \\
\text { FeI } \\
\end{array}$ & $\begin{array}{l}4312.35 \\
4312.86 \\
4312.99 \\
4314.08 \\
4314.31 \\
4314.97 \\
4315.08\end{array}$ & $\begin{array}{l}0.76 \\
0.72 \\
0.75\end{array}$ & $\begin{array}{l}-4.7 \\
6.42 \\
-6.19\end{array}$ & $\begin{array}{l}0.78 \\
0.81 \\
0.77\end{array}$ & $\begin{array}{c}30: \\
30.5: \\
23.7\end{array}$ & $\begin{array}{c}0.47 \\
\\
0.48 \\
0.5\end{array}$ & $\begin{array}{c}-5.7 \\
\\
-13.5 \\
-6.3\end{array}$ \\
\hline $\begin{array}{l}\operatorname{TiII}(94) \\
\mathrm{ZrII}(40)\end{array}$ & $\begin{array}{l}4316.79 \\
4317.32 \\
\end{array}$ & 0.95 & -5.73 & 0.93 & 27: & 0.82 & $-10.3:$ \\
\hline FeII & 4318.19 & 0.99 & -7.9 & & & & \\
\hline $\mathrm{CaI}(5)$ & 4318.66 & & & 0.95: & 19.4: & 0.94: & \\
\hline $\begin{array}{l}\text { FeII } \\
\text { FeII }(200)\end{array}$ & $\begin{array}{l}4319.41 \\
4319.68\end{array}$ & 0.97 & -10.65 & & & 0.9: & $-15.2:$ \\
\hline $\begin{array}{l}\operatorname{ScII}(15) \\
\operatorname{TiII}(41) \\
\text { FeII }\end{array}$ & $\begin{array}{l}4320.73 \\
4320.95 \\
4321.31 \\
\end{array}$ & 0.85 & -12.12 & 0.83 & 33 & 0.63 & -14.3 \\
\hline $\begin{array}{l}\text { ScII(15) } \\
\text { MnII } \\
\text { FeII } \\
\text { FeII } \\
\text { FeI(42) } \\
\text { MnII }\end{array}$ & $\begin{array}{l}4325.00 \\
4325.04 \\
4325.44 \\
4325.54 \\
4325.76 \\
4326.64\end{array}$ & $\begin{array}{l}0.82 \\
0.95\end{array}$ & $\begin{array}{c}-12.33 \\
-6.1\end{array}$ & 0.83 & 23.9: & 0.54 & $-12.8:$ \\
\hline $\begin{array}{l}\text { TiII }(94) \\
\operatorname{TiII}(41)\end{array}$ & $\begin{array}{l}4330.24 \\
4330.70\end{array}$ & 0.93 & -11.77 & 0.88 & 21 & $\begin{array}{l}0.82 \\
0.79 \\
\end{array}$ & $\begin{array}{c}-8.4: \\
-10.2:\end{array}$ \\
\hline $\mathrm{VII}(36)$ & 4331.55 & & & & & & \\
\hline
\end{tabular}




\begin{tabular}{|c|c|c|c|c|c|c|c|}
\hline \multirow[t]{2}{*}{ Ident } & \multirow[t]{2}{*}{$\bar{\lambda}$} & \multicolumn{2}{|c|}{$\alpha$ Cyg } & \multicolumn{2}{|r|}{3 Pup } & \multicolumn{2}{|c|}{ IRAS 05040} \\
\hline & & $r$ & $V_{r}(\mathrm{~km} / \mathrm{s})$ & $r$ & $V_{r}(\mathrm{~km} / \mathrm{s})$ & $r$ & $V_{r}(\mathrm{~km} / \mathrm{s}$ \\
\hline VII $(23)$ & 4331.79 & & & & & 0.92: & $-11:$ \\
\hline MgII & 4331.95 & 0.98 & -7.46 & & & & \\
\hline$\overline{\mathrm{FeII}(33)}$ & 4332.88 & & & & & $0.94:$ & $-13:$ \\
\hline FeI & 4337.05 & & & & & & \\
\hline TiII(94) & 4337.26 & & & & & & -6.2 \\
\hline $\operatorname{TiII}(20)$ & 4337.91 & 0.64 & -4.04 & & 30.4: & $0.5:$ & -2.1 : \\
\hline MnII & 4338.37 & & & & & & \\
\hline FeII & 4338.70 & & & & & & \\
\hline $\mathrm{HI}$ & 4340.46 & & & & & 0.64 & -48.3 \\
\hline & & 0.27 & -6.36 & 0.17 & 25.5 & 0.15 & $-11.4:$ \\
\hline Crll & 4341.07 & & & & & & \\
\hline TiII & 4341.36 & & & & & & \\
\hline CI & 4341.64 & & & & & & \\
\hline MnII & 4342.58 & & & & & & \\
\hline FeII & 4343.28 & 0.83 & -10.79 & & & & \\
\hline MnII & 4343.98 & & & & & & \\
\hline TiII(20) & 4344.28 & 0.82 & -16.07 & & 22.6: & 0.64 & -10.9 \\
\hline CrI & 4344.50 & & & & & & \\
\hline MnII & 4345.59 & & & & & & \\
\hline MnII & 4348.40 & 0.98 & -9.25 & & & 0.91: & \\
\hline TiII(94) & 4350.83 & & & & & 0.83 & -8.3 \\
\hline FeII $(27)$ & 4351.77 & & & 0.96 & -21.6 & & \\
\hline & 4351.77 & 0.47 & -4.4 & 0.58 & 32.1: & 0.29 & -4.9 \\
\hline CrI & 4351.81 & & & & & & \\
\hline $\operatorname{MgI}(14)$ & 4351.91 & & & & & & -9.1 \\
\hline FeI & 4352.73 & & & & & 0.88 & \\
\hline $\mathrm{FeII}(213)$ & 4354.34 & 0.95 & -5.23 & 0.94: & 33.1: & 0.87 & -11.9 \\
\hline ScII & 4354.60 & & & & & 0.94: & \\
\hline MnII & 4356.62 & 0.99 & -6.08 & & & & \\
\hline FeII & 4357.58 & 0.9 & -5.77 & 0.93 & 25.9: & 0.81 & -11.5 \\
\hline $\mathrm{FeI}(412)$ & 4358.51 & & & 0.97: & 19.8: & & \\
\hline$[\mathrm{FeII}] 7 \mathrm{~F}$ & 4359.34 & & & 1.02 & 4: & & \\
\hline ZrII & 4359.72 & 0.99 & -6.03 & & & & \\
\hline FeII & 4361.25 & 0.94 & -4.43 & 0.96: & 21.2: & \begin{tabular}{|l|l|}
0.89 \\
\end{tabular} & $-8.7:$ \\
\hline $\operatorname{NiII}(1)$ & 4362.10 & 0.91 & -4.44 & 0.94: & 21.4: & 0.78 & -12.1 \\
\hline CrII(179) & 4362.92 & & & & & 0.94: & -10.6 : \\
\hline MnII & 4363.26 & & & & & & \\
\hline MnII & 4365.22 & 0.99 & -5.65 & & & & \\
\hline$\overline{\mathrm{FeI}}(414)$ & 4367.59 & & & & & & \\
\hline TiII(104) & 4367.65 & 0.9 & -3.84 & 0.89: & & 0.72 & -11 \\
\hline CrII(37) & 4368.11 & & & & & 0.77 & $-8.6:$ \\
\hline OI & 4368.24 & & & & & & \\
\hline OI & 4368.26 & & & & & & \\
\hline$\overline{\mathrm{FeII}}(28)$ & 4369.41 & 0.84 & -4.94 & 0.89 & 33.2: & 0.64 & -8.6 \\
\hline FeI & 4369.77 & & & & & & \\
\hline$\overline{\mathrm{ZrII}(79)}$ & 4370.95 & 0.99 & -5.45 & & & & \\
\hline VII(36) & 4371.17 & & & & & & \\
\hline $\mathrm{CI}(14)$ & 4371.37 & & & 0.97: & & 0.94: & $-9:$ \\
\hline$\overline{\text { FeII(33) }}$ & 4372.22 & 0.99 & -4.76 & & & $0.97:$ & $-9.1:$ \\
\hline$\overline{\operatorname{ScII}(14)}$ & 4374.46 & & & & & 0.76 : & $-4.6:$ \\
\hline TiII(93) & 4374.82 & & & 0.87 & 24.4 & & $-13.7:$ \\
\hline YII & 4374.94 & & & & & & \\
\hline TiII & 4375.33 & & & & & & \\
\hline $\mathrm{FeI}(2)$ & 4375.93 & & & 0.97 & 20.7 & 0.98: & $-13:$ \\
\hline SiII & 4376.97 & 0.99 & -4.31 & & & 0.97: & \\
\hline SiII & 4376.99 & & & & & & \\
\hline
\end{tabular}




\begin{tabular}{|c|c|c|c|c|c|c|c|}
\hline \multirow[t]{2}{*}{ Ident } & \multirow[t]{2}{*}{$\lambda$} & \multicolumn{2}{|c|}{$\alpha \mathrm{Cyg}$} & \multicolumn{2}{|c|}{$3 \mathrm{Pup}$} & \multicolumn{2}{|c|}{ IRAS 05040} \\
\hline & & $r$ & $V_{r}(\mathrm{~km} / \mathrm{s})$ & $r$ & $V_{r}(\mathrm{~km} / \mathrm{s})$ & $r$ & $V_{r}(\mathrm{~km} / \mathrm{s})$ \\
\hline $\operatorname{MnII}(3)$ & 4377.77 & 0.99 & -9.78 & & & 0.98: & \\
\hline MnII & 4379.67 & & & & & & \\
\hline $\mathrm{ZrII}(88)$ & 4379.74 & 0.98 & -5.02 & & & $0.96:$ & \\
\hline$\overline{\mathrm{FeI}(41)}$ & 4383.55 & & & 1.01 & -24.3 & 0.57 & -9.3 \\
\hline & 4383.55 & 0.8 & -5.28 & 0.85 & 26.7: & & \\
\hline FeII & 4384.09 & & & & & & \\
\hline FeII(32) & 4384.32 & 0.76 & -2 & & & 0.53 & -3.6 \\
\hline $\operatorname{MgII}(10)$ & 4384.64 & & & & & & \\
\hline NiII & 4384.71 & & & & & & \\
\hline $\mathrm{ScII}$ & 4384.81 & & & & & & \\
\hline $\mathrm{FeII}(27)$ & 4385.39 & 0.42 & -5.13 & 0.73 & 25.6: & 0.39 & -3.8 \\
\hline FeII & 4386.59 & & & & & & \\
\hline $\operatorname{TiII}(104)$ & 4386.84 & 0.91 & -6.62 & 0.93 & 31.4: & 0.77 & -10.1 \\
\hline $\operatorname{HeI}(51)$ & 4387.93 & 0.95 & -3.77 & & & 0.92 & -21 \\
\hline $\mathrm{FeI}(830)$ & 4388.41 & & & & & 0.96: & \\
\hline MgII & 4390.51 & & & & & & \\
\hline $\operatorname{MgII}(10)$ & 4390.57 & 0.83 & -4.42 & & & 0.68 & $-15.5:$ \\
\hline $\mathrm{FeI}(414)$ & 4390.96 & & & & & & \\
\hline $\operatorname{TiII}(61)$ & 4391.02 & & & 0.89 & & 0.85 & $-12.5:$ \\
\hline MnII & 4393.38 & & & & & & \\
\hline $\operatorname{TiII}(51)$ & 4394.05 & 0.9 & -3.55 & 0.91 & 22.5 & 0.71 & -7.3 \\
\hline TiII(19) & 4395.03 & 0.6 & -4.97 & 0.63 & 30.6 & 0.34 & -4.2 \\
\hline $\operatorname{TiII}(61)$ & 4395.84 & & & & & 0.74 & -9.1 \\
\hline YII & 4398.01 & & & & & & \\
\hline $\operatorname{TiII}(61)$ & 4398.27 & 0.98 & -6.78 & 0.95: & & $0.92:$ & $-6.5:$ \\
\hline$\overline{T i I I}(51)$ & 4399.77 & 0.8 & -4.04 & 0.84 & $27.5:$ & 0.53 & -6 \\
\hline $\operatorname{ScII}(14)$ & 4400.39 & & & & & 0.77 & -11.1 \\
\hline $\operatorname{NiI}(86)$ & 4401.54 & & & 0.96 & 15.2: & 0.94: & \\
\hline FeII & 4402.88 & 0.95 & -4.66 & 0.96: & & 0.87 & $-13.2:$ \\
\hline $\mathrm{ZrII}(79)$ & 4403.35 & & & & & & \\
\hline $\mathrm{FeI}(41)$ & 4404.75 & 0.88 & -6.05 & 0.86 & $32:$ & 0.62 & -10.8 \\
\hline $\mathrm{VI}(22)$ & 4406.65 & & & 0.98: & 17.5: & 0.98: & \\
\hline $\operatorname{TiII}(61)$ & 4407.67 & 0.98 & -5 & 0.95: & 19.4: & 0.91 & $-9.4:$ \\
\hline $\mathrm{FeI}(68)$ & 4407.71 & & & & & & \\
\hline $\mathrm{FeI}(68)$ & 4408.42 & & & & & 0.98: & $-10:$ \\
\hline $\mathrm{VI}(22)$ & 4408.51 & & & & & & \\
\hline $\operatorname{TiII}(61)$ & 4409.40 & 0.96 & -2.54 & 0.96 & 24: & 0.88 & $-9.6:$ \\
\hline TiII(115) & 4411.07 & 0.92 & -5.39 & 0.92 & $33.2:$ & 0.77 & -9.5 \\
\hline TiII(61) & 4411.93 & 0.98 & -6.51 & 0.97: & & 0.94 & $-11.2:$ \\
\hline FeII $(32)$ & 4413.60 & 0.92 & -5 & 0.95 & 33.8: & 0.78 & -10.9 \\
\hline CoII & 4413.99 & & & & & & \\
\hline ZrII & 4414.54 & & & & & & \\
\hline $\mathrm{FeI}(41)$ & 4415.12 & 0.93 & 5.27 & & & 0.74 & -11.7 \\
\hline $\operatorname{ScII}(14)$ & 4415.56 & & & 0.9: & & 0.82 & $-12:$ \\
\hline $\operatorname{FeII}(27)$ & 4416.83 & 0.58 & -4.79 & 0.75 & 26.8 & 0.41 & -6.2 \\
\hline $\operatorname{TiII}(40)$ & 4417.71 & 0.78 & -5.84 & 0.82: & & 0.54 & -6.5 \\
\hline $\operatorname{TiII}(51)$ & 4418.33 & & & 0.84: & & 0.79 & -10.4 \\
\hline FeII & 4418.96 & & & & & & \\
\hline FeIII & 4419.60 & 0.98 & -5.82 & & & 0.96: & \\
\hline $\operatorname{ScII}(14)$ & 4420.67 & & & & & 0.98: & $-6:$ \\
\hline $\operatorname{TiII}(93)$ & 4421.94 & 0.96 & -5.54 & & & 0.84 & -9 \\
\hline $\mathrm{FeI}(350)$ & 4422.57 & & & 0.94: & & 0.95 & $-11.9:$ \\
\hline YII & 4422.59 & & & & & & \\
\hline $\operatorname{TiII}(61)$ & 4423.24 & & & 0.97: & & 0.96 & -13.3: \\
\hline $\mathrm{CaI}(4)$ & 4425.44 & & & 0.96: & 19.3 & 0.97: & \\
\hline $\mathrm{FeI}(2)$ & 4427.31 & & & 0.98 & 18.5: & $0.96:$ & $-9.5:$ \\
\hline
\end{tabular}




\begin{tabular}{|c|c|c|c|c|c|c|c|}
\hline \multirow[t]{2}{*}{ Ident } & \multirow[t]{2}{*}{$\lambda$} & \multicolumn{2}{|c|}{$\alpha \mathrm{Cyg}$} & \multicolumn{2}{|c|}{3 Pup } & \multicolumn{2}{|c|}{ IRAS 05040} \\
\hline & & $r$ & $V_{r}(\mathrm{~km} / \mathrm{s})$ & $r$ & $V_{r}(\mathrm{~km} / \mathrm{s})$ & $r$ & $\overline{V_{r}(\mathrm{~km} / \mathrm{s})}$ \\
\hline $\operatorname{TiII}(61)$ & 4427.88 & & & & & $0.83:$ & $-8.2:$ \\
\hline $\operatorname{MgII}(9)$ & 4427.99 & 0.94 & -5.22 & 0.98: & & & \\
\hline $\mathrm{ScII}(14)$ & 4431.37 & & & $0.98:$ & & & \\
\hline FeII & 4431.61 & 0.99 & -7.2 & & & & \\
\hline $\operatorname{TiII}(51)$ & 4432.11 & & & $0.97:$ & & & \\
\hline $\mathrm{FeI}(797)$ & 4432.57 & & & $0.98:$ & 19.6: & & \\
\hline $\mathrm{FeI}(830)$ & 4433.22 & & & 0.98: & 19.8: & & \\
\hline $\operatorname{MgII}(9)$ & 4433.99 & 0.91 & -4.93 & 0.94 & 23.9 & 0.78 & -14.6 \\
\hline $\mathrm{CaI}(4)$ & 4435.69 & & & 0.98: & 17.5: & 0.96 & \\
\hline $\operatorname{MgII}(19)$ & 4436.49 & 0.97 & -2.54 & & & $0.89:$ & $-16:$ \\
\hline $\operatorname{FeI}(567)$ & 4436.93 & & & $0.97:$ & 18: & & \\
\hline $\mathrm{HeI}(50)$ & 4437.55 & & & & & $0.97:$ & $-11:$ \\
\hline $\operatorname{TiII}(40)$ & 4441.73 & 0.96 & -4.66 & 0.95 & 18: & 0.85 & -9.8 \\
\hline $\mathrm{FeI}(68)$ & 4442.34 & & & & & 0.92 & $-11:$ \\
\hline ZrII & 4442.99 & & & & & & \\
\hline $\mathrm{FeI}$ & 4443.19 & & & & & & \\
\hline TiII(19) & 4443.79 & 0.66 & -4.22 & 0.74 & 37.4: & 0.41 & $-3.6:$ \\
\hline FeII & 4444.54 & & & & & & \\
\hline $\operatorname{TiII}(31)$ & 4444.55 & & & & & 0.8 & -11.6 \\
\hline$\overline{\mathrm{FeII}}(187)$ & 4446.24 & 0.98 & -5.4 & $0.99:$ & & 0.92 & $-12.5:$ \\
\hline $\mathrm{FeI}(828)$ & 4446.84 & & & 0.98: & 20: & & \\
\hline $\mathrm{FeI}(68)$ & 4447.72 & & & 0.97 & 33.1: & 0.93 & $-12.8:$ \\
\hline FeII $(222)$ & 4449.62 & 0.97 & -2.1 & & & $0.95:$ & $-11:$ \\
\hline TiII(19) & 4450.48 & & & 1.01 & -22.4 & & \\
\hline & 4450.48 & 0.84 & -5.64 & 0.85 & 28.1 & 0.63 & -7.2 \\
\hline FeII & 4451.55 & 0.88 & -5.44 & 0.93 & 23.6 & 0.78 & -13.5 \\
\hline $\operatorname{MnII}(22)$ & 4451.59 & & & $0.93:$ & 21: & & \\
\hline FeII & 4453.21 & 0.99 & -3.51 & & & & \\
\hline TiII(113) & 4453.32 & & & $0.97:$ & 18.7: & & \\
\hline VII(199) & 4453.34 & & & & & $0.97:$ & $-11:$ \\
\hline $\mathrm{CaI}(4)$ & 4454.78 & & & & & $0.93:$ & $-11:$ \\
\hline ZrII & 4454.79 & & & & & & \\
\hline FeII & 4455.27 & 0.91 & -5.42 & 0.93 & 23.7: & 0.81 & -13.7: \\
\hline $\mathrm{CaI}(4)$ & 4455.89 & & & 0.93 & 15.7: & & \\
\hline TiII(115) & 4456.65 & 0.98 & -5.07 & & & 0.92 & $-10.2:$ \\
\hline $\mathrm{CrII}(16)$ & 4456.84 & & & & & & \\
\hline NiI & 4459.03 & & & & & & \\
\hline $\mathrm{FeI}(68)$ & 4459.12 & 0.99 & -7.01 & 0.96 & 18.6 & 0.91: & -12.3 \\
\hline $\mathrm{VI}(21)$ & 4460.30 & & & 0.98 & 18.2 & & \\
\hline $\operatorname{ZrII}(67)$ & 4461.22 & & & & & & \\
\hline $\mathrm{FeII}(26)$ & 4461.44 & & & & & & \\
\hline FeII & 4461.71 & & & 1 & -34.3 & & \\
\hline & & 0.89 & -13.66 & 0.91: & 21.45: & 0.75 & -22.9 : \\
\hline Ce20) & 4463.41 & & & & & 0.97 & $-10:$ \\
\hline TiII(40) & 4464.45 & & & 1 & -24.4 & & \\
\hline & & 0.9 & -5.56 & 0.89 & $32.6:$ & 0.7 & -8.8 \\
\hline $\mathrm{CrII}(191)$ & 4465.73 & 0.99 & -3.82 & 0.97 & 21.1: & 0.95 & -8.4 \\
\hline $\mathrm{FeI}(350)$ & 4466.55 & 0.98 & -6.44 & 0.95 & 18.3 & 0.92 & $-11.9:$ \\
\hline $\mathrm{FeI}(992)$ & 4466.94 & & & $0.97:$ & 17: & & \\
\hline TiII(31) & 4468.51 & & & 1.02 & -23.6 & & \\
\hline & & 0.66 & -6.02 & 0.73 & $35.4:$ & 0.42 & $-5:$ \\
\hline TiII(18) & 4469.14 & & & & & $0.87:$ & $-6:$ \\
\hline $\mathrm{FeI}$ & 4469.38 & & & & & & \\
\hline $\operatorname{TiII}(40)$ & 4470.85 & & & $0.92:$ & & 0.8 & -9.5 \\
\hline $\operatorname{HeI}(14)$ & 4471.47 & 0.89 & -2.98 & 0.91: & & $0.86:$ & $-16.8:$ \\
\hline
\end{tabular}




\begin{tabular}{|c|c|c|c|c|c|c|c|}
\hline \multirow[t]{2}{*}{ Ident } & \multirow[t]{2}{*}{$\lambda$} & \multicolumn{2}{|c|}{$\alpha \mathrm{Cyg}$} & \multicolumn{2}{|c|}{$3 \mathrm{Pup}$} & \multicolumn{2}{|c|}{ IRAS 05040} \\
\hline & & $r$ & $V_{r}(\mathrm{~km} / \mathrm{s})$ & $r$ & $V_{r}(\mathrm{~km} / \mathrm{s})$ & $r$ & $V_{r}(\mathrm{~km} / \mathrm{s})$ \\
\hline $\mathrm{HeI}$ & 4471.49 & & & & & & \\
\hline $\mathrm{HeI}$ & 4471.68 & & & & & & \\
\hline$\overline{F e I I}(37)$ & 4472.93 & 0.84 & -5.94 & 0.89 & 28.4: & 0.65 & -8.9 \\
\hline$\overline{F e I I}(172)$ & 4474.19 & & & & & 0.94 & $-12.5:$ \\
\hline$\overline{\mathrm{FeI}}$ & 4476.02 & & & & & & \\
\hline $\operatorname{FeI}(350)$ & 4476.08 & & & 1.01 & -22.9 & & \\
\hline & & 0.99 & -7.44 & 0.96: & 18.3 & 0.91: & $-14.4:$ \\
\hline MnII & 4478.64 & 0.99 & -6.67 & & & 0.92 & $-11.5:$ \\
\hline FeII & 4480.69 & & & & & & \\
\hline MgII & 4481.13 & & & & & & \\
\hline MgII & 4481.15 & & & & & & \\
\hline $\operatorname{MgII}(4)$ & 4481.33 & 0.31 & -11.6 & 0.53 & 28.4: & 0.19 & -14.7 \\
\hline $\mathrm{FeI}(2)$ & 4482.18 & & & & & 0.91 & -9.5 \\
\hline $\mathrm{FeI}(68)$ & 4482.25 & & & & & & \\
\hline $\mathrm{FeI}(828)$ & 4484.23 & & & 0.98: & 19 & & \\
\hline $\mathrm{FeI}(830)$ & 4485.68 & & & 0.98 & 17.9: & & \\
\hline TiII(115) & 4488.32 & & & 0.99 & -25.6 & & \\
\hline & & 0.89 & -4.65 & 0.9 & 20.1 & 0.73 & -9.2 \\
\hline CrII & 4489.10 & & & & & & \\
\hline $\operatorname{FeII}(37)$ & 4489.18 & 0.69 & -5.26 & 0.79 & 27.2 & 0.53 & -6.8 \\
\hline FeII(37) & 4491.41 & 0.65 & -4.68 & 0.76 & 31.4: & 0.5 & -5.7 \\
\hline TiII(18) & 4493.51 & 0.96 & -4.98 & 0.96 & 21.3 & 0.87 & $-12:$ \\
\hline FeII & 4493.53 & & & & & & \\
\hline $\mathrm{FeI}(68)$ & 4494.56 & 0.98 & -5.87 & 0.96 & 19 & 0.89 & $-11.3:$ \\
\hline $\operatorname{CrI}(10)$ & 4496.85 & & & 0.97 & 20: & & \\
\hline $\mathrm{ZrII}(40)$ & 4496.96 & 0.99 & -5.54 & & & 0.97: & \\
\hline FeII & 4499.69 & 0.97 & -5.28 & & & 0.93: & $-13.5:$ \\
\hline $\operatorname{TiII}(31)$ & 4501.27 & & & 1.04 & -23 & & \\
\hline & & 0.69 & -4.91 & 0.76 & 33.4: & 0.45 & -3.9 \\
\hline $\mathrm{FeI}(555)$ & 4504.83 & & & 0.98: & 17.6: & & \\
\hline TiII & 4506.74 & & & & & & \\
\hline $\operatorname{FeII}(213)$ & 4507.10 & 0.97 & -4.71 & 0.98: & 19.5: & 0.95 & -8.5 : \\
\hline FeII(38) & 4508.29 & & & 1.01 & -25.5 & & \\
\hline & & 0.54 & -4.64 & 0.68 & $32.5:$ & 0.38 & $-4.5:$ \\
\hline NiII & 4509.27 & & & & & 0.95: & \\
\hline CrII & 4511.78 & & & & & & \\
\hline FeII & 4512.06 & 0.98 & -8.81 & & & 0.94: & \\
\hline VII & 4512.72 & & & & & & \\
\hline$\overline{F e I I}(37)$ & 4515.34 & & & 1.03 & -25.8 & & \\
\hline & & 0.58 & -4.29 & 0.72 & $32.7:$ & 0.41 & -4.3 \\
\hline FeII & 4515.61 & & & & & & \\
\hline CrII(191) & 4516.56 & & & & & $0.96:$ & $-9:$ \\
\hline TiII(18) & 4518.33 & 0.97 & -3.47 & 0.96: & & 0.91 & $-10.8:$ \\
\hline $\mathrm{VII}(212)$ & 4518.38 & & & & & & \\
\hline MnII & 4518.96 & & & & & & \\
\hline$\overline{F e I I}(37)$ & 4520.22 & & & 1.01 & -24.3 & & \\
\hline & & 0.59 & -4.32 & 0.74 & 32.3: & 0.42 & $-4.2:$ \\
\hline $\operatorname{FeII}(38)$ & 4522.63 & & & 1.01 & -25.3 & & \\
\hline & & 0.5 & -4.17 & 0.62 & 33.1: & 0.34 & -4.2 : \\
\hline SII & 4524.68 & & & & & & \\
\hline $\operatorname{TiII}(60)$ & 4524.68 & 0.97 & 11.62 & & & & \\
\hline SII & 4524.94 & & & & & 0.91 & $-12.8:$ \\
\hline $\mathrm{FeI}(826)$ & 4525.14 & & & 0.95: & 18.9 & & \\
\hline $\mathrm{FeI}(969)$ & 4526.45 & & & 0.97: & 20.5: & 0.94 & -9 \\
\hline FeII(171) & 4526.58 & & & & & & \\
\hline
\end{tabular}




\begin{tabular}{|c|c|c|c|c|c|c|c|}
\hline \multirow[t]{2}{*}{ Ident } & \multirow[t]{2}{*}{$\lambda$} & \multicolumn{2}{|c|}{$\alpha \mathrm{Cyg}$} & \multicolumn{2}{|c|}{$3 \mathrm{Pup}$} & \multicolumn{2}{|c|}{ IRAS 05040} \\
\hline & & $r$ & $V_{r}(\mathrm{~km} / \mathrm{s})$ & $r$ & $V_{r}(\mathrm{~km} / \mathrm{s})$ & $r$ & $V_{r}(\mathrm{~km} / \mathrm{s})$ \\
\hline $\mathrm{CaI}(36)$ & 4526.93 & & & 0.99: & 19: & & \\
\hline TiI(42) & 4527.32 & & & 0.98: & 20: & & \\
\hline $\mathrm{VII}(50)$ & 4528.49 & & & & & & \\
\hline $\mathrm{FeI}(68)$ & 4528.61 & & & 1.03 & -23.6 & & \\
\hline & & 0.95 & -9.29 & 0.94 & 19.2 & 0.78 & $-14:$ \\
\hline $\operatorname{TiII}(82)$ & 4529.47 & 0.92 & -5.26 & 0.93 & 28: & 0.78 & -8.4 \\
\hline $\mathrm{FeI}(39)$ & 4531.15 & & & 0.98: & 19.3: & $0.98:$ & \\
\hline $\mathrm{FeI}(555)$ & 4531.63 & & & 0.98: & 17.5: & & \\
\hline $\operatorname{VII}(212)$ & 4532.19 & & & 0.99: & 19.2: & $0.97:$ & $-9:$ \\
\hline TiII(50) FeII(37) & 4534.02 & & & 1.02 & -24 & & \\
\hline 4534.02 & 0.58 & -4.64 & 0.66 & 33: & 0.36 & -5.3 & \\
\hline MgII & 4534.28 & & & & & & \\
\hline MgII & 4534.30 & & & & & & \\
\hline $\mathrm{CrII}(39)$ & 4539.60 & 0.95 & -4.47 & 0.96 & $30.2:$ & 0.85 & $-9:$ \\
\hline $\mathrm{FeII}(38)$ & 4541.52 & 0.69 & -5.29 & $\begin{array}{l}1.02 \\
0.81\end{array}$ & $\begin{array}{l}-24.3 \\
30.7:\end{array}$ & 0.53 & -6.7 \\
\hline $\operatorname{TiII}(60)$ & 4544.02 & 0.98 & -6.05 & $\begin{array}{c}1.01 \\
0.97:\end{array}$ & $\begin{array}{l}-23 \\
20.6\end{array}$ & 0.9 & \\
\hline $\operatorname{TiII}(30)$ & 4545.13 & 0.97 & -6.42 & 0.96: & 25.4: & 0.9 & -9.5 \\
\hline $\mathrm{FeI}(755)$ & 4547.85 & & & 0.98: & 18.5: & & \\
\hline FeII & 4549.19 & & & & & & \\
\hline FeII(38) TiII(82) & 4549.54 & 0.42 & -8.2 & 0.51 & 29.7 & 0.32 & $-8:$ \\
\hline $\operatorname{TiII}(30)$ & 4552.29 & 0.98 & 0.8 & & & 0.92 & $-12.5:$ \\
\hline $\operatorname{TiI}(42)$ & 4552.46 & & & 0.97 & 20 & & \\
\hline SiIII & 4552.62 & & & & & & \\
\hline $\mathrm{BaII}(1)$ & 4554.03 & & & 1.02: & -22.5 & & \\
\hline & & 0.97 & -4.78 & 0.95: & 20.3 & 0.89 & $-12:$ \\
\hline $\operatorname{CrII}(44)$ & 4554.99 & 0.78 & -5.03 & 0.89: & 26.1: & 0.59 & -6.7 \\
\hline $\mathrm{FeII}(37)$ & 4555.89 & 0.54 & -4.62 & 0.67 & 29.7: & 0.37 & -4.2 \\
\hline $\operatorname{CrII}(44)$ & 4558.65 & & & 1.03 & -26.7 & & \\
\hline CrII & 4558.78 & 0.56 & -5.13 & 0.71 & $32.5:$ & 0.37 & -5.1 \\
\hline TiII(50) & 4563.76 & & & 1.02 & -23.3 & & \\
\hline $\mathrm{VII}(56)$ & 4564.58 & 0.7 & -4.98 & 0.75 & $34.2:$ & $\begin{array}{l}0.44 \\
0.89\end{array}$ & $\begin{array}{l}-4.1 \\
-9.3\end{array}$ \\
\hline $\operatorname{CrII}(39)$ & 4565.74 & 0.9 & -5.27 & 0.93 & $25.7:$ & 0.73 & -9.3 \\
\hline $\operatorname{TiII}(60)$ & 4568.31 & 0.99 & -5.93 & 0.97: & $23.2:$ & $0.94:$ & $-9.6:$ \\
\hline CoII & 4569.25 & 0.99 & -4.79 & & & 0.94 & -14.3 \\
\hline $\operatorname{TiII}(82)$ & 4571.97 & 0.63 & -4.71 & $\begin{array}{l}1.04 \\
0.71\end{array}$ & $\begin{array}{l}-24.4 \\
33.1:\end{array}$ & 0.4 & -3.6 \\
\hline $\mathrm{FeII}(38)$ & 5476.34 & 0.7 & -4.98 & $\begin{array}{l}1.03 \\
0.82 \\
\end{array}$ & $\begin{array}{c}-23.2 \\
33.2 \text { : }\end{array}$ & 0.52 & -6.2 \\
\hline $\mathrm{CaI}(23)$ & 4578.55 & & & 0.98 & 19.3 & & \\
\hline FeII & 4579.53 & & & & & 0.86 & $-13.5:$ \\
\hline $\mathrm{FeII}(26)$ & 4580.06 & & & 0.99 & -25.8 & & \\
\hline TiII & 4580.44 & 0.88 & -6.66 & 0.9 & 29.8: & 0.73 & -8.7 \\
\hline $\mathrm{CaI}(23)$ & 4581.40 & & & 0.98: & 20: & & \\
\hline $\operatorname{FeI}(555)$ & 4581.51 & & & 0.98: & 19.5: & & \\
\hline FeII(37) & 4582.84 & & & 1.02 & -24.8 & & \\
\hline $\operatorname{FeII}(37)$ & 4582.84 & 0.74 & -4.24 & 0.85 & 28.2 & 0.59 & -6.6 \\
\hline TiII & 4583.41 & & & & & & \\
\hline $\mathrm{FeII}(38)$ & 4583.84 & 0.46 & -4.49 & 0.55 & 29.4 & 0.31 & -5.7 \\
\hline FeII & 4584.00 & & & & & & \\
\hline $\mathrm{CaI}(23)$ & 4585.87 & & & 0.98 & 20.5 & & \\
\hline
\end{tabular}




\begin{tabular}{|c|c|c|c|c|c|c|c|}
\hline \multirow[t]{2}{*}{ Ident } & \multirow[t]{2}{*}{$\lambda$} & \multicolumn{2}{|c|}{$\alpha \mathrm{Cyg}$} & \multicolumn{2}{|c|}{$3 \mathrm{Pup}$} & \multicolumn{2}{|c|}{ IRAS 05040} \\
\hline & & $r$ & $\overline{V_{r}(\mathrm{~km} / \mathrm{s})}$ & $r$ & $V_{r}(\mathrm{~km} / \mathrm{s})$ & $r$ & $V_{r}(\mathrm{~km} / \mathrm{s})$ \\
\hline CrII & 4587.26 & & & & & 0.95 & -6.1 \\
\hline $\operatorname{CrII}(44)$ & 4588.20 & & & 1.02 & $-23.7:$ & & \\
\hline & & 0.6 & -4.74 & 0.74 & $32:$ & 0.41 & $-6:$ \\
\hline $\operatorname{CrII}(44)$ & 4589.90 & 0.87 & -3.12 & & & & \\
\hline $\operatorname{TiII}(50)$ & 4589.95 & & & 1.01 & $-21.4:$ & & \\
\hline & & & & 0.89 & 30.6: & 0.67 & -8.4 \\
\hline $\operatorname{CrII}(44)$ & 4592.05 & 0.78 & -4.77 & 0.87 & 28: & 0.59 & -7.1 \\
\hline $\operatorname{NiI}(98)$ & 4532.53 & & & 0.89: & & & \\
\hline $\mathrm{FeI}(39)$ & 4592.00 & & & & & & \\
\hline FeII & 4593.83 & 0.97 & -8.76 & 0.98: & & 0.91 & -14.5 \\
\hline FeII(38) & 4595.68 & & & & & & \\
\hline FeII & 4596.02 & 0.88 & -7.35 & 0.92 & 25.9 & 0.79 & -14.8 \\
\hline FeII(219) & 4598.49 & 0.96 & -5.18 & 0.98 & 29.8: & 0.92 & $-14.2:$ \\
\hline VII(50) & 4600.17 & & & 1.01 & $-20.4:$ & & \\
\hline & & 0.99 & -3.14 & 0.97: & 28.6: & 0.92 & $-10.1:$ \\
\hline NiI(98) & 4600.36 & & & & & & \\
\hline $\operatorname{CrI}(21)$ & 4600.75 & & & 0.98: & 18.6: & & \\
\hline FeII(43) & 4601.38 & 0.97 & -3.39 & 0.98: & 20.8: & 0.9 & $-13:$ \\
\hline $\mathrm{FeI}(39)$ & 4602.00 & & & 0.97: & 18.2: & & \\
\hline $\mathrm{FeI}(39)$ & 4602.94 & & & 0.98 & 19.3 & 0.98: & $-10:$ \\
\hline NiI(98) & 4604.99 & & & 0.99: & 19.5: & 0.97: & \\
\hline $\operatorname{VII}(56)$ & 4605.35 & & & 0.99: & & 0.97: & \\
\hline $\mathrm{FeI}(554)$ & 4607.65 & & & 0.99 & 19 & 0.97: & \\
\hline TiII(39) & 4609.26 & 0.99 & -4.83 & 0.99: & & 0.97: & \\
\hline FeII & 4610.59 & 0.99 & -4.09 & & & 0.95: & $-17.2:$ \\
\hline $\mathrm{FeI}(826)$ & 4611.28 & & & 0.98 & 19.1 & & \\
\hline $\operatorname{CrII}(44)$ & 4616.63 & & & 1.01 & -26.4 & & \\
\hline & & 0.81 & -5.43 & 0.88 & 32.2 : & 0.63 & -8.1 \\
\hline CrII(44) & 4618.80 & & & 1.02 & -23.3 & & \\
\hline & & 0.32 & -4.44 & 0.8 & 33.4: & 0.49 & -5.3 \\
\hline FeII & 4619.63 & & & & & & \\
\hline $\operatorname{FeII}(38)$ & 4620.52 & & & 1.01 & -21.8 & & \\
\hline & & 0.79 & -5.28 & 0.87 & 31.1: & 0.62 & -8.7 \\
\hline SiII & 4621.42 & & & & & 0.94 & -8.2 : \\
\hline SiII & 4621.72 & 0.97 & -14.57 & & & & \\
\hline $\operatorname{CrI}(32)$ & 4621.94 & & & 0.98: & & & \\
\hline $\operatorname{CrI}(233)$ & 4622.45 & & & 0.99: & 18.5: & & \\
\hline TiI(145) & 4623.10 & & & 0.99 & 17.9: & & \\
\hline $\mathrm{FeI}(554)$ & 4625.05 & & & $0.99:$ & 18.5 & & \\
\hline FeII(186) & 4625.89 & 0.96 & -5.22 & $0.97:$ & 23.6 & 0.9 & -12.9 \\
\hline $\operatorname{CrI}(21)$ & 4626.18 & & & 0.97 & 17.3: & & \\
\hline FeII & 4628.79 & & & & & & \\
\hline TiII & 4629.28 & & & & & & \\
\hline $\mathrm{FeII}(37)$ & 4629.34 & & & 1.02 & -27.1 & & \\
\hline & & 0.56 & -4.67 & 0.71 & 31.7: & 0.4 & -4.5 \\
\hline $\mathrm{FeII}(219)$ & 4631.87 & 0.99 & -6.29 & 0.99: & 21.7: & 0.94 & $-13.3:$ \\
\hline $\mathrm{FeI}(850)$ & 4632.83 & & & 0.99: & 21.4: & & \\
\hline $\operatorname{CrII}(44)$ & 4634.07 & & & 1.02 & -24.2 & & \\
\hline & 4634.07 & 0.72 & -4.68 & 0.82 & 33: & 0.53 & -5.6 \\
\hline FeII(186) & 4635.32 & 0.8 & -4.8 & 0.89 & 26.7 & 0.71 & -9.8 \\
\hline $\operatorname{TiII}(38)$ & 4636.32 & & & & & 0.97: & $-13.7:$ \\
\hline $\mathrm{FeI}(822)$ & 4638.02 & & & 0.97 & 19.6 & 0.89 & -13.3 \\
\hline FeII & 4638.05 & 0.96 & -5.99 & & & & \\
\hline FeII & 4640.81 & 0.98 & -6.53 & 0.99: & & 0.96 & -19.1 \\
\hline $\mathrm{FeI}(820)$ & 4643.47 & & & 0.99 & 19.5 & & \\
\hline
\end{tabular}




\begin{tabular}{|c|c|c|c|c|c|c|c|}
\hline \multirow[t]{2}{*}{ Ident } & \multirow[t]{2}{*}{$\lambda$} & \multicolumn{2}{|c|}{$\alpha \mathrm{Cyg}$} & \multicolumn{2}{|c|}{$3 \mathrm{Pup}$} & \multicolumn{2}{|c|}{ IRAS 05040} \\
\hline & & $r$ & $V_{r}(\mathrm{~km} / \mathrm{s})$ & $r$ & $V_{r}(\mathrm{~km} / \mathrm{s})$ & $r$ & $\overline{V_{r}(\mathrm{~km} / \mathrm{s})}$ \\
\hline $\operatorname{CrI}(21)$ & 4646.16 & & & 0.99: & 19.4: & 0.98: & $-11.5:$ \\
\hline $\mathrm{FeI}(409)$ & 4647.44 & & & 0.99 & 17.8 & 0.98: & $-12:$ \\
\hline NiI(98) & 4648.65 & & & & & & \\
\hline \multirow[t]{2}{*}{$\mathrm{FeII}(25)$} & 4648.94 & & & 1.01 & $-25.6:$ & & \\
\hline & & 0.97 & -6.78 & 0.98 & 23.1: & 0.87 & $-13.9:$ \\
\hline $\operatorname{CrI}(21)$ & 4651.29 & & & 0.99 & 19.9 & $0.98:$ & \\
\hline $\operatorname{CrI}(21)$ & 4652.16 & & & 0.985 & 20.3 & $0.96:$ & $-12.7:$ \\
\hline $\mathrm{FeI}(38)$ & 4654.50 & & & & & & \\
\hline $\mathrm{FeI}(554)$ & 4654.62 & & & $0.98:$ & 19.4: & $0.94:$ & $-13:$ \\
\hline SII & 4656.76 & \multirow{4}{*}{0.85} & \multirow{4}{*}{-3.45} & & \multirow{4}{*}{$\begin{array}{c}-23.4: \\
36:\end{array}$} & \multirow{4}{*}{0.68} & \multirow{4}{*}{$-7.2:$} \\
\hline FeII(43) & 4656.98 & & & 1.01 & & & \\
\hline & & & & 0.89 & & & \\
\hline TiII(59) & 4657.20 & & & & & & \\
\hline CoII & 4660.63 & 0.98 & -7.82 & & & $0.96:$ & \\
\hline FeII $(146)$ & 4660.93 & & & 0.99 & 18.5: & & \\
\hline AlII & 4663.05 & 0.91 & -3.33 & & & 0.87 & -15.8 \\
\hline $\operatorname{FeII}(44)$ & 4663.71 & 0.91 & -6.97 & 0.93 & 21.5 & 0.76 & -11.9 \\
\hline NiII & 4665.55 & 0.98 & 0.02 & & & 0.95 & -6.6 \\
\hline \multirow[t]{2}{*}{ FeII(37) } & 4666.76 & & & 1.01 & -25.9 & & \\
\hline & & 0.79 & -5.26 & 0.87 & 31.8: & 0.63 & -7.7 \\
\hline $\mathrm{FeI}(554)$ & 4668.14 & & & $0.98:$ & 18.9: & & \\
\hline $\mathrm{FeI}(821)$ & 4669.17 & & & $0.99:$ & 20.8: & & \\
\hline NI & 4669.89 & \multirow{4}{*}{0.89} & \multirow{4}{*}{-3.4} & & \multirow{4}{*}{$\begin{array}{c}-20.8: \\
36.2:\end{array}$} & \multirow{4}{*}{0.74} & \multirow{4}{*}{-7.5} \\
\hline $\mathrm{FeII}(25)$ & 4670.18 & & & 1.01 & & & \\
\hline & & & & 0.92 & & & \\
\hline $\operatorname{ScII}(24)$ & 4670.41 & & & & & & \\
\hline $\mathrm{FeI}(820)$ & 4673.17 & & & $0.99:$ & $22.6:$ & $0.96:$ & \\
\hline SiII & 4673.26 & 0.98 & -6.36 & & & & \\
\hline SiII & 4673.28 & & & & & & \\
\hline $\mathrm{FeI}(821)$ & 4678.85 & & & 0.985 & 19.4 & $0.94:$ & \\
\hline NiII & 4679.16 & 0.98 & -7.09 & 0.985: & 23.8: & & \\
\hline $\mathrm{FeI}(346)$ & 4683.56 & & & $0.99:$ & 18: & & \\
\hline NiI(98) & 4686.22 & & & $0.99:$ & 19.5: & & \\
\hline TiII & 4688.73 & 1 & -5.02 & & & 0.98 & -6.1 \\
\hline $\mathrm{FeI}(820)$ & 4690.14 & & & 0.99 & 18.6 & & \\
\hline $\mathrm{FeI}(409)$ & 4691.42 & & & 0.99 & 17.9: & & \\
\hline $\mathrm{FeI}$ & 4694.86 & & & $0.99:$ & 20.2: & & \\
\hline CrII(177) & 4697.60 & 0.99 & -7.07 & 0.99 & & 0.95 & -13.6 : \\
\hline TiII(59) & 4698.66 & & & $0.99:$ & 23.9: & $0.96:$ & 0.9: \\
\hline Vi1 & 4699.33 & & & 0.99 & 17.8: & & \\
\hline $\mathrm{FeI}(935)$ & 4700.16 & & & $0.99:$ & 18.5: & & \\
\hline $\operatorname{NiI}(235)$ & 4701.53 & & & $0.99:$ & 19.6: & & \\
\hline $\operatorname{MgI}(11)$ & 4702.99 & & & 1 & -23 & & \\
\hline & & 0.97 & -8.49 & 0.95 & 33.4: & 0.83 & -12 \\
\hline $\mathrm{FeI}(821)$ & 4704.95 & & & 0.99 & 20 & & \\
\hline $\mathrm{FeI}(752)$ & 4705.46 & & & 0.995 & 19.1: & & \\
\hline FeI(554) & 4707.28 & & & 0.99 & 18 & $0.97:$ & $-12:$ \\
\hline TiII(49) & 4708.66 & & & 1.01 & -20.3 & & \\
\hline & & 0.97 & -6.52 & 0.96 & 30.5: & 0.89 & $-6.5:$ \\
\hline $\mathrm{FeI}(409)$ & 4708.67 & & & 0.99 & 17.7 & & \\
\hline HeI & 4713.14 & 0.95 & -1.45 & & & & \\
\hline $\mathrm{HeI}$ & 4713.16 & & & & & & \\
\hline $\operatorname{FeII}(26)$ & 4713.17 & & & 1 & $-28:$ & & \\
\hline & 4713.17 & & & 0.98 & 33: & 0.94 & $-9:$ \\
\hline NiI(98) & 4714.41 & $\overline{1}$ & -7.79 & 0.985 & 20.1 & 0.94 & $-12:$ \\
\hline
\end{tabular}




\begin{tabular}{|c|c|c|c|c|c|c|c|}
\hline \multirow[t]{2}{*}{ Ident } & \multirow[t]{2}{*}{$\lambda$} & \multicolumn{2}{|c|}{$\alpha \mathrm{Cyg}$} & \multicolumn{2}{|c|}{$3 \mathrm{Pup}$} & \multicolumn{2}{|c|}{ IRAS 05040} \\
\hline & & $r$ & $V_{r}(\mathrm{~km} / \mathrm{s})$ & $r$ & $V_{r}(\mathrm{~km} / \mathrm{s})$ & $r$ & $\overline{V_{r}(\mathrm{~km} / \mathrm{s})}$ \\
\hline $\mathrm{NiI}(98)$ & 4715.77 & & & 0.99 & 18.7 & & \\
\hline $\begin{array}{l}\text { SII } \\
\text { CaII }\end{array}$ & $\begin{array}{l}4716.27 \\
4716.74\end{array}$ & 0.99 & -4.33 & & & & \\
\hline $\operatorname{CrI}(186)$ & 4718.42 & & & 0.99 & 18.1 & & \\
\hline $\begin{array}{l}\text { TiII } \\
\text { FeII }(54) \\
\text { CaII } \\
\end{array}$ & $\begin{array}{l}4719.52 \\
4720.15 \\
4721.02\end{array}$ & 0.99 & -6.95 & 0.99 & 16.8 & $\begin{array}{l}0.97: \\
0.95:\end{array}$ & $-7.8:$ \\
\hline $\mathrm{ZnI}(2)$ & 4722.16 & & & 0.99 & 18.2 & & \\
\hline CrII & 4723.34 & 0.99 & -5.69 & & & 0.94 & -11.1 \\
\hline $\operatorname{MnII}(5)$ & 4727.84 & 0.99 & -4.71 & & & 0.95 & -11 \\
\hline $\operatorname{MnII}(5)$ & 4730.36 & & & & & 0.94 & $-7.1:$ \\
\hline FeII $(43)$ & 4731.45 & 0.77 & -4.91 & $\begin{array}{l}1.02 \\
0.85\end{array}$ & $\begin{array}{l}-22: \\
31.5:\end{array}$ & 0.6 & -6.9 \\
\hline $\mathrm{FeI}(38)$ & 4733.60 & & & 0.99 & 18.8: & & \\
\hline $\begin{array}{l}\mathrm{FeI}(554) \\
\mathrm{CrII}\end{array}$ & $\begin{array}{l}4736.77 \\
4737.00\end{array}$ & 0.99 & 2.7 & 0.98 & 20.6 & 0.94 & -5.2 \\
\hline $\operatorname{MnII}(5)$ & 4738.29 & 0.98 & -5.2 & 0.99 & 27.4 & 0.94 & -9.1 \\
\hline $\begin{array}{l}\text { MgII } \\
\text { MgII }\end{array}$ & $\begin{array}{l}4739.59 \\
4739.71\end{array}$ & 0.96 & -2.95 & 0.99 & & 0.89 & -12 \\
\hline $\mathrm{FeI}(346)$ & 4741.53 & & & 0.995: & 19.9: & & \\
\hline $\mathrm{FeI}(821)$ & 4745.80 & & & 0.99: & $20.8:$ & & \\
\hline $\operatorname{MnI}(16)$ & 4754.04 & & & 0.985 & 18.6 & & \\
\hline $\mathrm{NiI}(141)$ & 4754.76 & & & 0.99: & 18: & & \\
\hline $\operatorname{MnII}(5)$ & 4755.73 & 0.95 & -5.46 & $\begin{array}{c}1 \\
0.97\end{array}$ & $\begin{array}{c}-25.9 \\
30.6:\end{array}$ & 0.85 & -9.2 \\
\hline $\mathrm{FeI}(634)$ & 4757.58 & & & 0.995 & 18.9 & & \\
\hline $\operatorname{TiI}(233)$ & 4759.27 & & & 0.99 & 18.8 & & \\
\hline FeII(169) & 4760.15 & & & & & $0.96:$ & $-11:$ \\
\hline $\begin{array}{l}\mathrm{CrII}(176) \\
\operatorname{MnI}(21)\end{array}$ & $\begin{array}{l}4761.43 \\
4761.53\end{array}$ & 0.99 & -3.71 & $\begin{array}{l}0.99 \\
0.98\end{array}$ & $\begin{array}{c}26 \\
18.2\end{array}$ & $0.96:$ & \\
\hline $\operatorname{MnI}(21)$ & 4762.37 & & & & & 0.93: & $-12.8:$ \\
\hline $\operatorname{TiII}(17)$ & 4762.78 & 0.98 & -11.09 & 0.97 & & 0.9 & $-14.8:$ \\
\hline $\operatorname{TiII}(48)$ & 4763.88 & 0.97 & -4.28 & & & 0.89 & $-6.9:$ \\
\hline TiII(48) & 4764.52 & & & 0.97: & & & \\
\hline $\operatorname{MnII}(5)$ & 4764.73 & 0.95 & -6.68 & & & 0.85 & -14.4 \\
\hline CrII & 4765.11 & & & & & & \\
\hline $\operatorname{MnI}(21)$ & 4766.42 & & & 0.99 & 18.2 & & \\
\hline $\mathrm{FeI}$ & $\overline{4768.35}$ & & & 0.99 & 19.5: & & \\
\hline $\mathrm{CI}(6)$ & 4770.02 & & & & & 0.94 & -12 \\
\hline $\mathrm{CI}(6)$ & 4771.74 & 0.99 & -8.91 & 0.98 & & 0.86 & $-14:$ \\
\hline $\mathrm{OI}$ & 4773.76 & 0.99 & -3.93 & & & 0.95 & -13.2 \\
\hline$[\mathrm{FeII}] 20 \mathrm{~F}$ & 4774.74 & & & 1.01 & 27: & & \\
\hline $\mathrm{CI}(6)$ & 4775.89 & & & & & 0.94 & $-13.6:$ \\
\hline $\operatorname{TiII}(92)$ & 4779.99 & 0.91 & -6.08 & $\begin{array}{l}1.01 \\
0.91 \\
\end{array}$ & $\begin{array}{c}-24.8 \\
31.3:\end{array}$ & 0.74 & -8.3 \\
\hline $\operatorname{MnI}(16)$ & 4783.43 & 1 & -1.91 & 0.99 & 19.3 & 0.97: & $-12:$ \\
\hline $\mathrm{FeI}(588)$ & 4788.76 & & & 0.99 & 18.7 & & \\
\hline $\mathrm{FeI}(753)$ & 4783.65 & & & 0.99 & 19.4 & & \\
\hline $\operatorname{TiII}(17)$ & 4798.52 & 0.98 & -3.95 & $\begin{array}{l}1.03 \\
0.98 \\
1.02\end{array}$ & $\begin{array}{c}-18.6 \\
26.8 \\
71.6\end{array}$ & 0.92: & $-8:$ \\
\hline $\mathrm{FeI}(1042)$ & 4800.65 & & & 0.99: & 17.5: & & \\
\hline $\mathrm{FeI}(888)$ & 4802.88 & & & 0.99 & 19.8 & & \\
\hline $\operatorname{TiII}(92)$ & 4805.09 & 0.86 & -5.43 & \begin{tabular}{|l|}
1.02 \\
0.88 \\
\end{tabular} & $\begin{array}{c}-24.4 \\
32.6:\end{array}$ & 0.67 & -7.9 \\
\hline
\end{tabular}




\begin{tabular}{|c|c|c|c|c|c|c|c|}
\hline \multirow[t]{2}{*}{ Ident } & \multirow[t]{2}{*}{$\lambda$} & \multicolumn{2}{|c|}{$\alpha \mathrm{Cyg}$} & \multicolumn{2}{|c|}{$3 \mathrm{Pup}$} & \multicolumn{2}{|c|}{ IRAS 05040} \\
\hline & & $r$ & $V_{r}(\mathrm{~km} / \mathrm{s})$ & $r$ & $V_{r}(\mathrm{~km} / \mathrm{s})$ & $r$ & $V_{r}(\mathrm{~km} / \mathrm{s})$ \\
\hline TiII & 4806.32 & & & & & & \\
\hline MnII & 4806.82 & 0.99 & -3.95 & 0.985 & 27.2 & 0.95 & $-5.3:$ \\
\hline $\mathrm{ZnI}(2)$ & 4810.53 & & & 0.99: & 18.8: & & \\
\hline FeII(169) & 4810.75 & 0.99 & -5.87 & 0.99 & 28.9: & 0.95 & -13.9 \\
\hline $\mathrm{CrII}(30)$ & 4812.34 & & & 1 & -23.7 & & \\
\hline $\mathrm{CrII}(30)$ & 4812.34 & 0.91 & -4.99 & 0.93 & 33.1: & 0.76 & -8.2 \\
\hline VII(197) & 4813.95 & & & & & 0.97: & $-13:$ \\
\hline$[\mathrm{FeII}] 20 \mathrm{~F}$ & 4814.53 & & & 1.02: & 33: & & \\
\hline SII(9) & 4815.55 & 0.97 & -3.33 & 0.99 & 36.5 & 0.93 & -17.1 \\
\hline TiI(126) & 4820.41 & & & 0.99 & 19.9: & & \\
\hline FeII & 4820.83 & 0.99 & -7.95 & & & 0.96 & $-8.2:$ \\
\hline $\operatorname{MnI}(16)$ & 4823.52 & & & & & 0.94 & $-14.9:$ \\
\hline $\mathrm{CrII}(30)$ & 4824.13 & & & 1.01 & -26.7 & & \\
\hline & & 0.66 & -4.39 & 0.78 & $32.3:$ & 0.47 & $-4.3:$ \\
\hline FeII(30) & 4825.74 & 0.99 & -5.97 & & & 0.94 & $-10.3:$ \\
\hline FeII & 4826.68 & & & & & $0.94:$ & $-15:$ \\
\hline $\mathrm{NiI}(131)$ & 4829.03 & & & 0.99 & 19.3 & $0.98:$ & \\
\hline $\operatorname{CrI}(31)$ & 4829.37 & & & 0.99: & 17.1: & & \\
\hline FeII & 4831.20 & & & & & 0.96: & $-13:$ \\
\hline FeII(30) & 4833.20 & 0.98 & -5.48 & 0.99 & 32.3 & 0.92 & $-11.5:$ \\
\hline $\mathrm{CrII}(30)$ & 4836.23 & & & 1.01 & -25.8 & & \\
\hline & & 0.9 & -8.59 & 0.93 & 32.6: & 0.73 & -8.4 \\
\hline $\mathrm{FeII}(30)$ & 4840.00 & 0.98 & -8.59 & 0.98 & 32.1 & 0.94 & $-11.3:$ \\
\hline VII(248) & 4842.50 & & & & & $0.97:$ & $-13:$ \\
\hline FeII & 4843.30 & & & & & $0.97:$ & $-17:$ \\
\hline MnII & 4847.61 & & & & & 0.92 & $-5.9:$ \\
\hline $\mathrm{CrII}(30)$ & 4848.24 & & & 0.99 & -23.8 & & \\
\hline & & 0.72 & -3.73 & 0.8 & 33.4: & 0.52 & $-4.8:$ \\
\hline TiII & 4849.17 & & & & & & \\
\hline MgII & 4851.07 & & & & & & \\
\hline $\operatorname{MgII}(25)$ & 4851.10 & 0.96 & -6.26 & & & 0.89: & $-15.7:$ \\
\hline YII & 4854.86 & & & & & & \\
\hline NiI & 4855.41 & & & & & & \\
\hline $\mathrm{FeII}(25)$ & 4855.55 & & & & & 0.83: & $-6:$ \\
\hline TiII & 4855.91 & & & & & & \\
\hline $\operatorname{CrII}(30)$ & 4856.19 & 0.89 & -6.53 & & & $0.74:$ & $-10:$ \\
\hline FeI & 4859.74 & & & & & & \\
\hline CrII & 4860.20 & & & & & & \\
\hline FeII & 4860.68 & & & & & & \\
\hline SiII & 4861.07 & & & & & & \\
\hline $\mathrm{HI}$ & 4861.32 & & & 0.74 & -46.9 & 1.02 & -47.3 \\
\hline & & 0.38 & -9.21 & 0.2 & 27.5 & 0.25 & $-8.9:$ \\
\hline & & & & 0.87 & 96.6 & & \\
\hline $\mathrm{CrII}(30)$ & 4864.33 & 0.72 & -5.92 & & 23.6: & 0.54 & -5.1 \\
\hline TiII(29) & 4865.61 & & & & & & \\
\hline $\mathrm{FeII}(25)$ & 4871.28 & & & 0.97 & $-22.4:$ & & \\
\hline & & 0.93 & -5.61 & & 34: & 0.8 & $-10.9:$ \\
\hline $\mathrm{FeI}(318)$ & 4871.32 & & & & 20 & & \\
\hline $\mathrm{FeI}(318)$ & 4872.14 & & & & & 0.9 & $-14.1:$ \\
\hline TiII(114) & 4874.01 & 0.94 & -5.47 & $0.93:$ & 29: & 0.82 & -10.2 \\
\hline $\operatorname{CrII}(30)$ & 4876.40 & & & 1.01 & -22.9 & & \\
\hline & & 0.76 & -3.56 & 0.83 & 33.4 & 0.58 & -4.7 \\
\hline CrII & 4876.47 & & & & & & \\
\hline $\mathrm{FeI}(318)$ & 4878.21 & 0.99 & -3.59 & & & 0.95 & -9.4 \\
\hline FeII & 4883.29 & & & & & 0.98: & $-11.5:$ \\
\hline VII(209) & 4883.41 & & & & & & \\
\hline
\end{tabular}




\begin{tabular}{|c|c|c|c|c|c|c|c|}
\hline \multirow[t]{2}{*}{ Ident } & \multirow[t]{2}{*}{$\lambda$} & \multicolumn{2}{|c|}{$\alpha \mathrm{Cyg}$} & \multicolumn{2}{|c|}{$3 \mathrm{Pup}$} & \multicolumn{2}{|c|}{ IRAS 05040} \\
\hline & & $r$ & $\overline{V_{r}(\mathrm{~km} / \mathrm{s})}$ & $r$ & $V_{r}(\mathrm{~km} / \mathrm{s})$ & $r$ & $\overline{V_{r}(\mathrm{~km} / \mathrm{s})}$ \\
\hline $\mathrm{YII}(22)$ & 4883.68 & 0.97 & -19.35 & 0.97: & 17.4: & $0.95:$ & \\
\hline $\operatorname{CrII}(30)$ & 4884.61 & 0.93 & -5.71 & 0.95 & 28.4 & $0.8:$ & $-9.8:$ \\
\hline $\mathrm{FeI}(1066)$ & 4886.40 & & & 0.99 & 19.1 & $0.97:$ & \\
\hline $\mathrm{FeI}(318)$ & 4890.76 & & & 1.02 & -20 & & \\
\hline & & 0.98 & -5.7 & 0.98 & 19.9 & 0.87 & $-14.7:$ \\
\hline $\operatorname{FeI}(318)$ & 4891.49 & 0.97 & -5.81 & 0.95 & 23 & 0.81 & -12.2 \\
\hline$\overline{\mathrm{FeII}}(36)$ & 4893.82 & & & 1 & -21 & & \\
\hline & & 0.96 & -6.11 & 0.97 & 30.7 & 0.87 & $-12.7:$ \\
\hline $\mathrm{FeII}(36)$ & 4893.82 & & & 1 & 76 & & \\
\hline $\mathrm{BaII}(3)$ & 4899.93 & & & 0.97 & 37 & & \\
\hline $\mathrm{YII}(22)$ & 4900.12 & & & 1.01 & $-24.8:$ & & \\
\hline & & 0.99 & -6.9 & 0.97 & 25.5: & 0.96 & $-12:$ \\
\hline CrII(190) & 4901.62 & 0.97 & -5.99 & 0.97 & 36.4 & 0.91 & -11.3 \\
\hline $\mathrm{FeI}(318)$ & 4903.31 & & & 0.98 & 18.5 & 0.94 & -11.9 \\
\hline $\operatorname{NiI}(129)$ & 4904.42 & & & 0.99 & 18.3 & $0.95:$ & \\
\hline FeII & 4908.15 & 0.98 & -5.11 & 0.99 & & 0.93 & $-17:$ \\
\hline $\mathrm{FeI}(687)$ & 4910.02 & & & & & $0.95:$ & $-15:$ \\
\hline $\mathrm{FeI}(1068)$ & 4910.57 & & & & & 0.96 & -12 \\
\hline TiII(114) & 4911.19 & & & 1.01 & -25.2 & & \\
\hline & & 0.91 & -4.81 & 0.92 & 32.4 & 0.76 & -8.2 \\
\hline CrII(190) & 4912.46 & 0.97 & -6.66 & & & 0.92 & -9.9 \\
\hline $\operatorname{FeII}(218)$ & 4913.29 & 0.95 & -6.21 & & & 0.89 & $-10.5:$ \\
\hline $\operatorname{NiI}(132)$ & 4913.98 & & & 0.98: & 17.7: & & \\
\hline $\mathrm{FeI}(1066)$ & 4917.23 & & & $0.99:$ & 19.4: & $0.97:$ & \\
\hline $\mathrm{FeI}(318)$ & 4918.99 & 0.98 & -5.09 & 0.96 & 29.8 & 0.87 & -12.3 \\
\hline $\operatorname{CrII}(36)$ & 4920.23 & & & & & & \\
\hline MnII & 4920.44 & & & & & & \\
\hline FeI (318) & 4920.50 & 0.94 & -6.08 & 0.94 & 31.4 & 0.77 & -12.7 \\
\hline $\operatorname{HeI}(48)$ & 4921.93 & 0.93 & -1.82 & 0.97 & 31.2 & 0.93 & $-10.5:$ \\
\hline $\mathrm{FeII}(42)$ & 4923.93 & & & 1 & $-34.4:$ & & \\
\hline & & 0.43 & -4.46 & 0.44 & 31.6 & 0.35 & -9 \\
\hline SII & 4924.11 & & & & & & \\
\hline FeII & 4924.92 & & & & & & \\
\hline SII & 4925.34 & 0.98 & -6.88 & & & 0.95 & -3.4 \\
\hline FeI & 4927.87 & & & 0.99 & 18.8 & & \\
\hline $\mathrm{CI}(13)$ & 4932.05 & & & & & 0.95 & -14 \\
\hline$\overline{\mathrm{BaII}}(1)$ & 4934.08 & 0.98 & -6.08 & $\begin{array}{l}1.01 \\
0.96 \\
1.01\end{array}$ & $\begin{array}{c}-19.6: \\
30.7: \\
77.4:\end{array}$ & 0.94 & $-13.5:$ \\
\hline NI & 4935.11 & 0.99 & -6.2 & & & & \\
\hline $\mathrm{NiI}(114)$ & 4937.35 & & & 0.99 & 18.3 & $0.98:$ & \\
\hline $\mathrm{FeI}(318)$ & 4938.81 & 0.99 & -8.1 & 0.99 & 20.1 & $0.96:$ & $-11.4:$ \\
\hline $\mathrm{FeI}(1065)$ & 4939.24 & & & $0.99:$ & 19.3: & $0.98:$ & $-9:$ \\
\hline $\mathrm{FeI}(16)$ & 4939.69 & & & 0.99: & 18.7: & 0.98: & $-7:$ \\
\hline CrII(36) & 4941.03 & & & & & $0.99:$ & $-12:$ \\
\hline $\mathrm{FeI}(687)$ & 4946.39 & & & 0.99 & 19 & & \\
\hline FeII & 4948.10 & 0.98 & -3.98 & & & $0.94:$ & $-13.8:$ \\
\hline FeII & 4948.79 & 0.97 & -6.44 & 0.98 & & 0.92 & $-16.5:$ \\
\hline $\mathrm{FeI}(687)$ & 4950.11 & & & $0.995:$ & 20.1: & 0.97 & \\
\hline FeII & 4951.58 & 0.94 & -5.28 & $0.98:$ & 27.8: & 0.86 & -16.2 \\
\hline CrII & 4952.79 & 0.98 & -7.23 & & & 0.92 & $-15.8:$ \\
\hline FeII(168) & 4953.99 & 0.97 & -4.65 & 0.98 & & 0.93 & -11.5 \\
\hline $\operatorname{BaII}(10)$ & 4957.15 & & & & & & \\
\hline $\mathrm{FeI}(318)$ & 4957.30 & & & & & & \\
\hline $\operatorname{FeI}(318)$ & 4957.60 & & & 1.01 & -27.2 & & \\
\hline
\end{tabular}




\begin{tabular}{|c|c|c|c|c|c|c|c|}
\hline \multirow[t]{2}{*}{ Ident } & \multirow[t]{2}{*}{$\lambda$} & \multicolumn{2}{|c|}{$\alpha \mathrm{Cyg}$} & \multicolumn{2}{|c|}{$3 \mathrm{Pup}$} & \multicolumn{2}{|c|}{ IRAS 05040} \\
\hline & & $r$ & $V_{r}(\mathrm{~km} / \mathrm{s})$ & $r$ & $V_{r}(\mathrm{~km} / \mathrm{s})$ & $r$ & $V_{r}(\mathrm{~km} / \mathrm{s})$ \\
\hline FeII & 4958.83 & 0.93 & -8.97 & 0.92 & 24.6 & $\begin{array}{c}0.74 \\
0.98:\end{array}$ & -11.6 \\
\hline FeII & 4958.82 & 0.99 & -4.7 & & & & \\
\hline FeI(1097) & 4962.57 & & & 0.99 & 19.8 & & \\
\hline VII(221) & 4963.75 & & & & & & \\
\hline DIB & 4963.90 & & & & & 0.97: & -8.6 \\
\hline $\operatorname{CrII}(36)$ & 4964.34 & & & & & 0.98: & $-12.5:$ \\
\hline $\mathrm{VII}(209)$ & 4965.40 & & & & & $0.98:$ & $-10:$ \\
\hline$\overline{\mathrm{VII}(29)}$ & 4966.08 & & & & & & \\
\hline $\mathrm{FeI}(687)$ & 4966.09 & & & 0.99: & 19.8: & 0.96: & $-11:$ \\
\hline $\mathrm{OI}(14)$ & 4967.38 & & & & & $0.96:$ & $-8:$ \\
\hline & & 0.99 & -6.33 & & & 0.93: & $-14.7:$ \\
\hline FeI(1067) & 4967.90 & & & 0.98: & 19.8: & & \\
\hline $\mathrm{OI}(14)$ & 4968.79 & 0.98 & -5.38 & & & $0.92:$ & $-13.5:$ \\
\hline $\mathrm{FeI}(1066)$ & 4969.92 & & & $0.99:$ & 18.7: & & \\
\hline FeII & 4971.30 & & & & & $0.95:$ & $-15.5:$ \\
\hline $\mathrm{FeI}(984)$ & 4973.11 & & & $0.99:$ & 18.7: & & \\
\hline FeII & 4974.22 & & & & & $0.98:$ & $-16:$ \\
\hline FeII & 4977.04 & 0.96 & -5.15 & 0.98 & 29.3 & 0.89 & $-15.8:$ \\
\hline FeII & 4977.92 & & & & & $0.97:$ & $-15.5:$ \\
\hline $\mathrm{NaI}$ & 4978.54 & & & 0.99 & 20.7: & & \\
\hline $\mathrm{NiI}(112)$ & 4980.17 & & & 0.99 & 20.7: & & \\
\hline TiI(38) & 4981.74 & & & 0.99 & 19.2 & & \\
\hline FeI(1067) & 4982.50 & 0.99 & 0.74 & 0.98 & 20.3 & $0.96:$ & $-8:$ \\
\hline $\mathrm{NaI}$ & 4982.81 & & & 0.98 & 20 & & \\
\hline $\mathrm{FeI}(1067)$ & 4983.25 & & & 0.98 & 20.2 : & & \\
\hline $\mathrm{FeI}$ & 4983.85 & & & 0.975 & 19.6 & & \\
\hline NiI & 4984.11 & & & 0.97 & 19.7 & & \\
\hline FeII & 4984.49 & 0.95 & -5.91 & 0.97 & 24.1 & 0.9 & $-16.4:$ \\
\hline $\mathrm{FeI}(984)$ & 4985.26 & & & & & $0.96:$ & $-12:$ \\
\hline $\mathrm{FeI}(318)$ & 4985.55 & & & & & & \\
\hline $\mathrm{FeI}(1066)$ & 4988.95 & & & 0.99: & 19: & 0.98: & $-12:$ \\
\hline FeII & 4990.51 & 0.94 & -3.66 & & & 0.87 & -15.2 \\
\hline $\operatorname{FeII}(25)$ & 4991.13 & 0.97 & -4.37 & $0.96:$ & 20.75: & 0.88 & -7.3 \\
\hline FeII & 4991.46 & & & & & & \\
\hline $\operatorname{SII}(7)$ & 4991.97 & & & & & $0.92:$ & $-14:$ \\
\hline & 4992.02 & 0.97 & -7.7 & & & & \\
\hline $\operatorname{FeII}(36)$ & 4993.36 & & & 1.02 & -21.5 & & \\
\hline & & 0.88 & -4.87 & $\begin{array}{l}0.93 \\
1.01\end{array}$ & $\begin{array}{c}29.5: \\
76.5\end{array}$ & 0.75 & -7.2 \\
\hline TiII & 4996.37 & 0.99 & -4.57 & & & & \\
\hline NiI(14) & 4998.23 & & & 0.99: & 19.6: & & \\
\hline FeII & 4999.18 & 0.99 & -6.2 & & & $0.95:$ & $-17:$ \\
\hline TiI(38) & 4999.51 & & & 0.985 & 19.3 & & \\
\hline FeII $(25)$ & 5000.74 & 0.98 & -2.7 & & & 0.93 & $-6.8:$ \\
\hline CaII & 5001.48 & & & & & & \\
\hline $\mathrm{FeI}$ & 5001.86 & & & & & & \\
\hline FeII & 5001.96 & 0.93 & -6.06 & 0.92 & 32 & 0.74 & -15.5 \\
\hline FeII & 5004.20 & 0.91 & -5.66 & 0.96 & 32.8: & 0.8 & -16 \\
\hline $\mathrm{FeI}(984)$ & 5005.71 & & & 0.99 & 20 & & \\
\hline $\mathrm{FeI}(318)$ & 5006.12 & & & & & $0.93:$ & $-13.3:$ \\
\hline FeII & 5006.84 & 0.98 & -4.69 & & & 0.94 & $-15:$ \\
\hline $\mathrm{FeI}$ & 5007.28 & & & 0.98 & 18.7 & & \\
\hline FeII & 5007.45 & & & & & 0.92 & -9.4 \\
\hline FeII & 5007.74 & 0.97 & -12.12 & & & & \\
\hline
\end{tabular}




\begin{tabular}{|c|c|c|c|c|c|c|c|}
\hline \multirow[t]{2}{*}{ Ident } & \multirow[t]{2}{*}{$\lambda$} & \multicolumn{2}{|c|}{$\alpha \mathrm{Cyg}$} & \multicolumn{2}{|c|}{$3 \mathrm{Pup}$} & \multicolumn{2}{|c|}{ IRAS 05040} \\
\hline & & $r$ & $V_{r}(\mathrm{~km} / \mathrm{s})$ & $r$ & $V_{r}(\mathrm{~km} / \mathrm{s})$ & $r$ & $\overline{V_{r}(\mathrm{~km} / \mathrm{s})}$ \\
\hline FeII & 5009.00 & 0.99 & -0.11 & & & 0.97: & $-14:$ \\
\hline SII & 5009.57 & & & & & & \\
\hline TiII(113) & 5010.21 & 0.97 & -9.63 & 0.97 & 27.3 & 0.91 & $-12.3:$ \\
\hline $\mathrm{FeI}(16)$ & 5012.08 & & & & & 0.97 & $-7:$ \\
\hline TiII & 5013.33 & & & & & & \\
\hline $\operatorname{TiII}(71)$ & 5013.68 & 0.97 & 5.95 & $\begin{array}{l}1.03 \\
0.97 \\
1.01\end{array}$ & $\begin{array}{c}-24.5 \\
34.4: \\
73:\end{array}$ & 0.9 & $-7.9:$ \\
\hline SII & 5014.04 & & & & & & \\
\hline $\mathrm{FeI}(965)$ & 5014.95 & & & & 18.7: & 0.96 & -12.9 : \\
\hline $\operatorname{HeI}(4)$ & 5015.68 & & & & & & \\
\hline FeII & 5015.76 & 0.93 & -6.36 & 0.97 & 27.3 & 0.9 & -16.7 \\
\hline NiI & 5017.57 & & & & & & \\
\hline $\mathrm{FeII}(42)$ & 5018.44 & & & 1.01 & $-41:$ & & \\
\hline$\Gamma-\mathrm{T}$ & & 0.43 & -4.42 & 0.39 & 28: & 0.39 & -10 \\
\hline $\begin{array}{l}\text { FeII } \\
\text { VII(232) }\end{array}$ & 5019.46 & & & & & 0.89: & \\
\hline $\mathrm{CaII}(15)$ & 5019.97 & & & & & 0.84: & -16.7 : \\
\hline $\mathrm{OI}$ & 5020.22 & & & & & & \\
\hline FeII & 5021.59 & 0.97 & -8.08 & 0.99 & 29.5 & 0.92 & \\
\hline TilI & 5022.05 & & & & & & \\
\hline FeII & 5022.42 & & & & & 0.91: & $-19:$ \\
\hline FeII & 5022.79 & 0.95 & -14.92 & 0.97 & 22.3 & 0.89 & $-18.1:$ \\
\hline CrII & 5024.52 & 0.99 & -2.96 & & & 0.96 & -7.3 \\
\hline $\begin{array}{l}\text { FeII } \\
\text { SII }\end{array}$ & $\begin{array}{l}5026.81 \\
5027.22\end{array}$ & 0.97 & -2.36 & & & 0.93: & $-14.3:$ \\
\hline FeII & 5030.63 & 0.91 & -1 & & & 0.83 & -15.8 \\
\hline $\operatorname{ScII}(23)$ & 5031.02 & & & $\begin{array}{l}1.02 \\
0.94 \\
1.01\end{array}$ & $\begin{array}{c}-25: \\
23.2: \\
74:\end{array}$ & $0.87:$ & $-13:$ \\
\hline $\operatorname{SII}(7)$ & 5032.43 & & & & & & \\
\hline FeII & 5032.71 & 0.94 & -10.3 & 0.97 & 28 & 0.89 & $-18.1:$ \\
\hline $\mathrm{NiI}$ & 5035.36 & & & & & & \\
\hline FeII & 5035.71 & 0.89 & -4.98 & & & 0.8 & -16.1 \\
\hline $\operatorname{FeII}(36)$ & 5036.92 & 0.98 & -11.1 & & & 0.93 & $-12.3:$ \\
\hline $\mathrm{CI}(4)$ & 5039.67 & & & & & 0.95 & $-16:$ \\
\hline SiII(5) & 5041.02 & 0.73 & -5.43 & 0.88: & 33.4: & 0.63 & -13.8 \\
\hline FeII & 5045.11 & 0.97 & -4.6 & & & 0.92 & $-16.3:$ \\
\hline $\begin{array}{l}\text { FeII } \\
\operatorname{HeI}(47)\end{array}$ & $\begin{array}{l}5047.64 \\
5047.71\end{array}$ & 0.95 & -5.19 & 0.97: & $28:$ & 0.89 & -16.2 \\
\hline $\mathrm{FeI}$ & 5049.82 & 1 & -3.98 & & & & \\
\hline $\mathrm{I}(12)$ & 5052.17 & 0.99 & -6.57 & & & 0.88 & -14.5 \\
\hline $\begin{array}{l}\text { SiII(5) } \\
\text { SiII }\end{array}$ & $\begin{array}{l}5055.98 \\
5056.32\end{array}$ & 0.66 & -0.91 & 0.81: & 39.3: & 0.55 & -7.5 \\
\hline FeII & 5060.26 & & & & & 0.97: & $-17.5:$ \\
\hline FeII & 5061.72 & 0.95 & -6.28 & 0.98: & 33.5 & 0.87 & -17 \\
\hline FeII & 5062.93 & & & & & $0.98:$ & $-19:$ \\
\hline $\begin{array}{l}\text { FeI(1094) } \\
\text { FeII }\end{array}$ & $\begin{array}{l}5065.02 \\
5065.42\end{array}$ & 0.98 & -1.81 & & & 0.93 & $-11.4:$ \\
\hline FeII & 5067.89 & 0.98 & -6.35 & & & 0.93 & $-17.5:$ \\
\hline FeII & 5070.58 & & & & & & \\
\hline FeII & 5070.90 & 0.94 & -5.94 & & & 0.86 & -16.6 \\
\hline TilI(113) & 5072.28 & 0.95 & -3.74 & 0.97: & & 0.87 & -8.8 \\
\hline $\begin{array}{l}\text { FeII } \\
\text { FeI }(1094)\end{array}$ & $\begin{array}{l}5074.05 \\
5074.75\end{array}$ & 0.97 & -9.58 & & & $\begin{array}{l}\text { 0.93: } \\
0.96:\end{array}$ & $\begin{array}{c}-16.2: \\
-14:\end{array}$ \\
\hline
\end{tabular}




\begin{tabular}{|c|c|c|c|c|c|c|c|}
\hline \multirow[t]{2}{*}{ Ident } & \multirow[t]{2}{*}{$\lambda$} & \multicolumn{2}{|c|}{$\alpha \mathrm{Cyg}$} & \multicolumn{2}{|c|}{$3 \mathrm{Pup}$} & \multicolumn{2}{|c|}{ IRAS 05040} \\
\hline & & $r$ & $V_{r}(\mathrm{~km} / \mathrm{s})$ & $r$ & $V_{r}(\mathrm{~km} / \mathrm{s})$ & $r$ & $\overline{V_{r}(\mathrm{~km} / \mathrm{s})}$ \\
\hline FeII & 5075.76 & 0.95 & -2.61 & & & 0.89 & -16 \\
\hline $\mathrm{CrII}(201)$ & 5076.15 & & & & & & \\
\hline $\mathrm{FeI}(1089)$ & 5076.27 & & & & & & \\
\hline FeII & 5076.61 & & & & & & \\
\hline$\overline{\mathrm{FeI}(1092)}$ & 5078.98 & & & & & & \\
\hline $\operatorname{FeI}(66)$ & 5079.23 & & & & & $0.97:$ & $-12:$ \\
\hline NiI(143) & 5080.54 & & & & & $0.97:$ & $-13:$ \\
\hline NiI(194) & 5081.11 & & & & & $0.97:$ & $-10.7:$ \\
\hline $\operatorname{FeII}(221)$ & 5081.90 & & & & & & \\
\hline FeII & 5082.23 & 0.97 & -7.05 & & & 0.93 & $-16:$ \\
\hline FeII & 5086.31 & & & & & 0.94 & $-8.5:$ \\
\hline FeII & 5087.30 & 0.97 & -6.62 & & & 0.91 & -17.8 \\
\hline $\mathrm{YII}(20)$ & 5087.42 & & & & & & \\
\hline FeII & 5089.21 & 0.96 & -4.4 & 0.985: & 33.9: & 0.9 & -15.4 \\
\hline FeII & 5089.42 & & & & & & \\
\hline FeII & 5093.47 & & & & & & \\
\hline FeII & 5093.58 & & -5.56 & & & 0.87 & -15.2 \\
\hline FeII & 5093.77 & & & & & & \\
\hline FeII & 5094.90 & & & & & 0.97 & -11.5 \\
\hline FeII & 5097.27 & & & & & 0.84 & -12.9 \\
\hline $\operatorname{CrII}(24)$ & 5097.31 & 0.92 & -8.39 & 0.95 & 26.6: & & \\
\hline $\mathrm{FeI}(66)$ & 5098.70 & 0.98 & -8.35 & & & 0.94 & -14.4 \\
\hline FeII & 5100.61 & & & & & & \\
\hline FeII & 5100.66 & & & & & & \\
\hline FeII & 5100.73 & & & 1 & -22 : & & \\
\hline & & 0.82 & -4.42 & 0.89 & $33.5:$ & 0.73 & -10 \\
\hline FeII & 5100.85 & & & & & & \\
\hline NdIII & 5102.43 & 0.99 & -3.54 & & & & \\
\hline SII & 5103.33 & 1 & -5.34 & & & & \\
\hline FeII & 5106.11 & 0.98 & -4.36 & & & 0.92 & $-14.2:$ \\
\hline FeII & 5107.12 & & & & & & \\
\hline $\mathrm{FeI}(16)$ & 5107.50 & 0.98 & -5.05 & 0.99 & 22.3 & $0.95:$ & -18.4 : \\
\hline $\operatorname{FeI}(36)$ & 5107.64 & & & & & & \\
\hline $\mathrm{FeI}(1)$ & 5110.42 & & & $0.99:$ & 18.5: & $0.98:$ & \\
\hline FeII & 5112.99 & 0.99 & -6.64 & 0.99: & 29: & $0.97:$ & $-10:$ \\
\hline FeII & 5115.06 & 0.99 & -5.9 & & & $0.97:$ & \\
\hline FeII & 5117.03 & 0.97 & -4.33 & 0.99: & $26.2:$ & 0.93 & -17.2 \\
\hline FeII(35) & 5120.35 & 0.96 & -4.8 & 0.97 & 33.1: & 0.88 & -9.7 \\
\hline FeII & 5123.19 & & & & & 0.94 & $-11.5:$ \\
\hline YII(21) & 5123.21 & 0.98 & -2.45 & & & & \\
\hline$\overline{\mathrm{FeI}}(1090)$ & 5125.12 & 0.99 & -6.38 & & & $0.97:$ & $-14.8:$ \\
\hline $\operatorname{NiI}(160)$ & 5125.23 & & & & & & \\
\hline NdIII & 5127.04 & & & & & & \\
\hline $\mathrm{FeI}(16)$ & 5127.36 & & & & & 0.95 & $-15:$ \\
\hline FeII(167) & 5127.87 & 0.93 & -7.03 & 0.97 & & 0.87 & -10.9 \\
\hline $\operatorname{TiII}(86)$ & 5129.15 & & & 1.01 & $-15.3:$ & & \\
\hline & & 0.92 & -5.65 & $\begin{array}{l}0.93 \\
1.02\end{array}$ & $\begin{array}{l}30.2: \\
74.7:\end{array}$ & 0.79 & $-7:$ \\
\hline $\operatorname{TiII}(86)$ & 5131.30 & & & & & & \\
\hline $\operatorname{FeI}(66)$ & 5131.47 & & & & & 0.97 & $-16:$ \\
\hline FeII $(35)$ & 5132.67 & & & 1.02 & $-19:$ & & \\
\hline & & 0.95 & -5.16 & $\begin{array}{l}0.97 \\
1.01\end{array}$ & $\begin{array}{c}24.7: \\
71:\end{array}$ & 0.86 & \\
\hline $\mathrm{FeI}$ & 5133.69 & & & & & 0.93 & -12.9 \\
\hline FeII $(35)$ & 5136.80 & & & 1.01 & -21.5 & & \\
\hline
\end{tabular}




\begin{tabular}{|c|c|c|c|c|c|c|c|}
\hline \multirow[t]{2}{*}{ Ident } & \multirow[t]{2}{*}{$\lambda$} & \multicolumn{2}{|c|}{$\alpha \mathrm{Cyg}$} & \multicolumn{2}{|c|}{$3 \mathrm{Pup}$} & \multicolumn{2}{|c|}{ IRAS 05040} \\
\hline & & $r$ & $V_{r}(\mathrm{~km} / \mathrm{s})$ & $r$ & $V_{r}(\mathrm{~km} / \mathrm{s})$ & $r$ & $\overline{V_{r}(\mathrm{~km} / \mathrm{s})}$ \\
\hline $\mathrm{CrII}(201)$ & 5137.09 & 0.96 & -1.11 & 0.98 & 35.9 & 0.9 & $-13.1:$ \\
\hline$\overline{\mathrm{FeI}(318)}$ & 5139.25 & & & & & & \\
\hline $\mathrm{FeI}$ & 5139.46 & & & 1.01 & -22.8 & & \\
\hline $\mathrm{FeI}$ & 5139.46 & 0.98 & -9.82 & 0.98 & 28.4 & 0.9 & $-16.2:$ \\
\hline FeII & 5140.69 & & & & & 0.97 & $-16:$ \\
\hline FeII & 5143.88 & & & & & & \\
\hline FeII & 5144.36 & & & 1 & -26.8 & & \\
\hline & & 0.95 & -8.16 & 0.97 & 26.1 & 0.87 & -15.1 \\
\hline FeII & 5145.77 & & & & & & \\
\hline FeII & 5145.82 & & & & & & \\
\hline $\mathrm{FeII}(35)$ & 5146.13 & & & 1.01 & -22.8 & & \\
\hline & & 0.93 & -12.62 & 0.96 & 26.4 & 0.83 & -14.4 \\
\hline & & & & 1.01 & 75.1 & & \\
\hline FeII & 5148.94 & & & & & $0.92:$ & $-13:$ \\
\hline FeII & 5149.47 & 0.92 & 8.01 & 0.97 & 22.6 & 0.85 & -17.1 \\
\hline FeII & 5150.49 & & & & & 0.94: & \\
\hline FeII & 5150.94 & 0.97 & -18.93 & & & 0.92: & $-15.5:$ \\
\hline FeII & 5152.97 & & & & & $0.95:$ & $-17:$ \\
\hline $\operatorname{CrII}(24)$ & 5153.50 & & & & & $0.86:$ & $-10.3:$ \\
\hline $\operatorname{TiII}(70)$ & 5154.07 & & & 1.01 & -22.9 & & \\
\hline & & 0.93 & -1.12 & 0.94 & 30.7 & & \\
\hline $\operatorname{FeII}(35)$ & 5154.41 & & & & & 0.81: & $-18:$ \\
\hline FeIII & 5156.12 & 0.99 & -2.54 & & & & \\
\hline FeII & 5157.28 & 0.98 & -2.78 & & & 0.94: & $-16.1:$ \\
\hline FeII & 5157.51 & & & & & & \\
\hline FeII & 5158.07 & & & & & $0.97:$ & \\
\hline$[\mathrm{FeII}] 19 \mathrm{~F}$ & 5158.78 & & & 1.05 & 27.8 & & \\
\hline FeII & 5159.91 & & & & & 0.98: & $-9.5:$ \\
\hline FeII(187) & 5160.84 & 0.94 & -1.71 & 0.96 & 38.2 & 0.85 & $-7.3:$ \\
\hline FeII(35) & 5161.18 & & & & & & \\
\hline FeI & 5162.27 & 1 & -2.34 & & & 0.94: & $-13.8:$ \\
\hline FeII & 5163.01 & & & & & & \\
\hline FeII & 5163.58 & 0.99 & -8.85 & & & 0.96: & $-15.5:$ \\
\hline $\mathrm{FeI}(1089)$ & 5165.41 & & & & & & \\
\hline FeII & 5166.56 & & & & & 0.91 & -15.7 \\
\hline $\operatorname{MgI}(2)$ & 5167.32 & & & 1.02 & $-15.9:$ & & \\
\hline & & 0.9 & -3.6 & 0.91 & 33.4: & 0.67 & -6.8 \\
\hline $\mathrm{FeI}$ & 5167.49 & & & & & & \\
\hline $\mathrm{FeI}(1)$ & 5168.90 & & & & & 0.51 & -11.9 \\
\hline FeII(42) & 5169.03 & 0.42 & -4.63 & 0.32 & 31.8: & 0.41 & -9.3 \\
\hline FeII & 5170.13 & & & & & $0.96:$ & $-12.3:$ \\
\hline FeII & 5170.78 & & & & & $0.95:$ & $-18.3:$ \\
\hline $\mathrm{FeI}(36)$ & 5171.60 & & & & & 0.92 & $-10:$ \\
\hline FeII & 5171.64 & & & & & & \\
\hline $\operatorname{MgI}(2)$ & 5172.68 & 0.83 & -3.87 & & & 0.6 & $-6.2:$ \\
\hline FeII & 5177.02 & & & & & $0.93:$ & \\
\hline FeII & 5177.39 & 0.96 & -10.39 & 0.98: & 30: & $0.92:$ & $-15:$ \\
\hline FeII & 5178.37 & 0.99 & -1.96 & & & 0.97: & \\
\hline FeII & 5180.31 & 0.97 & -4.59 & & & 0.91 & -14.8 \\
\hline $\operatorname{FeII}(35)$ & 5180.53 & & & & & & \\
\hline $\operatorname{MgI}(2)$ & 5183.60 & & & 1.04 & $-23.5:$ & & \\
\hline & & 0.79 & -5.32 & 0.84 & 27.1: & 0.57 & -7.3 \\
\hline TiII & 5183.71 & & & & & & \\
\hline SiII & 5185.52 & & & & & & \\
\hline SiII & 5185.56 & & & & 0.94 & & \\
\hline
\end{tabular}




\begin{tabular}{|c|c|c|c|c|c|c|c|}
\hline \multirow[t]{2}{*}{ Ident } & \multirow[t]{2}{*}{$\lambda$} & \multicolumn{2}{|c|}{$\alpha \mathrm{Cyg}$} & \multicolumn{2}{|c|}{$3 \mathrm{Pup}$} & \multicolumn{2}{|c|}{ IRAS 05040} \\
\hline & & $r$ & $V_{r}(\mathrm{~km} / \mathrm{s})$ & $r$ & $V_{r}(\mathrm{~km} / \mathrm{s})$ & $r$ & $\overline{V_{r}(\mathrm{~km} / \mathrm{s})}$ \\
\hline TiII(86) & 5185.90 & 0.93 & -7.67 & $\begin{array}{l}1.02 \\
0.93 \\
1.01\end{array}$ & $\begin{array}{c}-22.8: \\
29.3: \\
75.7:\end{array}$ & 0.82 & $-7:$ \\
\hline FeII & 5186.87 & 0.99 & -9.08 & & & 0.95 & -16.5 \\
\hline $\begin{array}{l}\operatorname{TiII}(70) \\
\mathrm{CaI}(49)\end{array}$ & $\begin{array}{l}5188.68 \\
5188.84\end{array}$ & 0.86 & -4.38 & $\begin{array}{l}1.04 \\
0.88 \\
1.01\end{array}$ & $\begin{array}{c}-19.1 \\
31.4 \\
77.6\end{array}$ & 0.69 & $-4.9:$ \\
\hline FeII & 5190.01 & & & & & 0.97: & $-14:$ \\
\hline $\begin{array}{l}\mathrm{CrII}(24) \\
\mathrm{FeI}(383) \\
\mathrm{FeI}(383)\end{array}$ & $\begin{array}{l}5191.43 \\
5191.45 \\
5192.34\end{array}$ & $\begin{array}{l}0.98 \\
0.98\end{array}$ & $\begin{array}{l}-2.2 \\
5.75\end{array}$ & 0.97: & 20.8: & $\begin{array}{l}0.88: \\
0.92:\end{array}$ & $\begin{array}{l}-8.6: \\
-9.4:\end{array}$ \\
\hline FeII & 5193.74 & & & & & 0.97: & $-14:$ \\
\hline $\begin{array}{l}\text { FeII } \\
\text { FeI }(36) \\
\text { FeI }\end{array}$ & $\begin{array}{l}5194.89 \\
5194.94 \\
5195.47\end{array}$ & 0.98 & -4.7 & & & $\begin{array}{l}0.93: \\
0.96:\end{array}$ & $\begin{array}{c}-13: \\
-13.4:\end{array}$ \\
\hline$\overline{\mathrm{FeII}}(49)$ & 5197.58 & 0.64 & -4 & $\begin{array}{l}1.06 \\
0.77 \\
1.02\end{array}$ & $\begin{array}{c}-21.4 \\
29.2 \\
76.6\end{array}$ & 0.49 & -2.2 : \\
\hline $\begin{array}{l}\text { FeI(66) } \\
\text { FeII }\end{array}$ & $\begin{array}{l}5198.72 \\
5199.12\end{array}$ & 0.96 & -6.31 & 0.99: & 27.9: & 0.9 & -14.7 \\
\hline $\begin{array}{l}\text { YII } \\
\text { FeII } \\
\text { SII } \\
\text { FeII }\end{array}$ & $\begin{array}{l}5200.41 \\
5200.80 \\
5201.02 \\
5201.45\end{array}$ & 0.98 & -1.17 & & & $\begin{array}{l}0.94 \\
0.97:\end{array}$ & $\begin{array}{c}-10.6: \\
-16:\end{array}$ \\
\hline $\begin{array}{l}\text { FeI(66) } \\
\text { SiII } \\
\text { FeII }\end{array}$ & $\begin{array}{l}5202.34 \\
5202.41 \\
5202.58\end{array}$ & 0.99 & -0.44 & & & 0.94 & -16 \\
\hline $\begin{array}{l}\text { FeII } \\
\text { NdIII } \\
\text { CrI( } 7) \\
\end{array}$ & $\begin{array}{l}5203.64 \\
5203.92 \\
5204.51 \\
\end{array}$ & 0.97 & -2.94 & & & $\begin{array}{l}0.92 \\
0.95 \\
\end{array}$ & $\begin{array}{l}-15.9: \\
-14.9:\end{array}$ \\
\hline $\begin{array}{l}\text { YII(20) } \\
\text { CrI }\end{array}$ & $\begin{array}{l}5205.72 \\
5206.04\end{array}$ & 0.99 & -10.07 & & & 0.91 & $-11.7:$ \\
\hline $\begin{array}{l}\operatorname{CrI}(7) \\
\operatorname{FeI}(553)\end{array}$ & $\begin{array}{l}5208.43 \\
5208.60\end{array}$ & 0.98 & -4.9 & 0.96 & 29.4 & 0.89 & -10.2 \\
\hline $\begin{array}{l}\operatorname{TiI}(4) \\
\operatorname{CrII}(24) \\
\operatorname{TiII}(103)\end{array}$ & $\begin{array}{l}5210.39 \\
5210.87 \\
5211.53\end{array}$ & $\begin{array}{l}0.97 \\
0.98\end{array}$ & $\begin{array}{l}-11.6 \\
-6.25\end{array}$ & & & $\begin{array}{c}0.93: \\
0.91\end{array}$ & $\begin{array}{c}-12.9: \\
-9.2\end{array}$ \\
\hline SII & 5212.62 & 0.99 & -2.66 & & & & \\
\hline FeII & 5213.96 & 0.97 & -3.6 & & & 0.92 & $-14.3:$ \\
\hline $\begin{array}{l}\text { FeII } \\
\text { FeII } \\
\text { FeII }\end{array}$ & $\begin{array}{l}5215.35 \\
5215.76 \\
5215.84\end{array}$ & & & & & $\begin{array}{c}0.89 \\
0.9 \\
0.91\end{array}$ & $\begin{array}{l}-17.1 \\
-22.3 \\
-12.3:\end{array}$ \\
\hline $\begin{array}{l}\text { FeII } \\
\text { FeII } \\
\text { FeI(553) }\end{array}$ & $\begin{array}{l}5216.85 \\
5216.86 \\
5217.39\end{array}$ & 0.89 & -5.66 & 0.97: & 19.9: & 0.83 & $-14.4:$ \\
\hline FeII & 5218.84 & 0.97 & -8.15 & & & 0.94 & $-20.1:$ \\
\hline$[\mathrm{FeII}] 19 \mathrm{~F}$ & 5220.06 & & & 1.01: & & & \\
\hline FeII & 5222.36 & 0.99 & -6.23 & & & & \\
\hline $\begin{array}{l}\text { FeII } \\
\text { FeII }\end{array}$ & $\begin{array}{l}5223.26 \\
5223.81\end{array}$ & 0.98 & -3.74 & & & $\begin{array}{l}0.94: \\
0.96:\end{array}$ & $\begin{array}{c}-13.7: \\
-20:\end{array}$ \\
\hline $\begin{array}{l}\text { FeII } \\
\text { FeII } \\
\text { TiII(70) } \\
\text { FeI(383) }\end{array}$ & $\begin{array}{l}5225.30 \\
5225.99 \\
5226.54 \\
5226.86\end{array}$ & 0.88 & -4.47 & $\begin{array}{l}1.03 \\
0.91\end{array}$ & $\begin{array}{c}-20.3: \\
31.7:\end{array}$ & $\begin{array}{l}0.93 \\
0.92 \\
0.71\end{array}$ & $\begin{array}{l}-15: \\
-13: \\
-3.2\end{array}$ \\
\hline
\end{tabular}




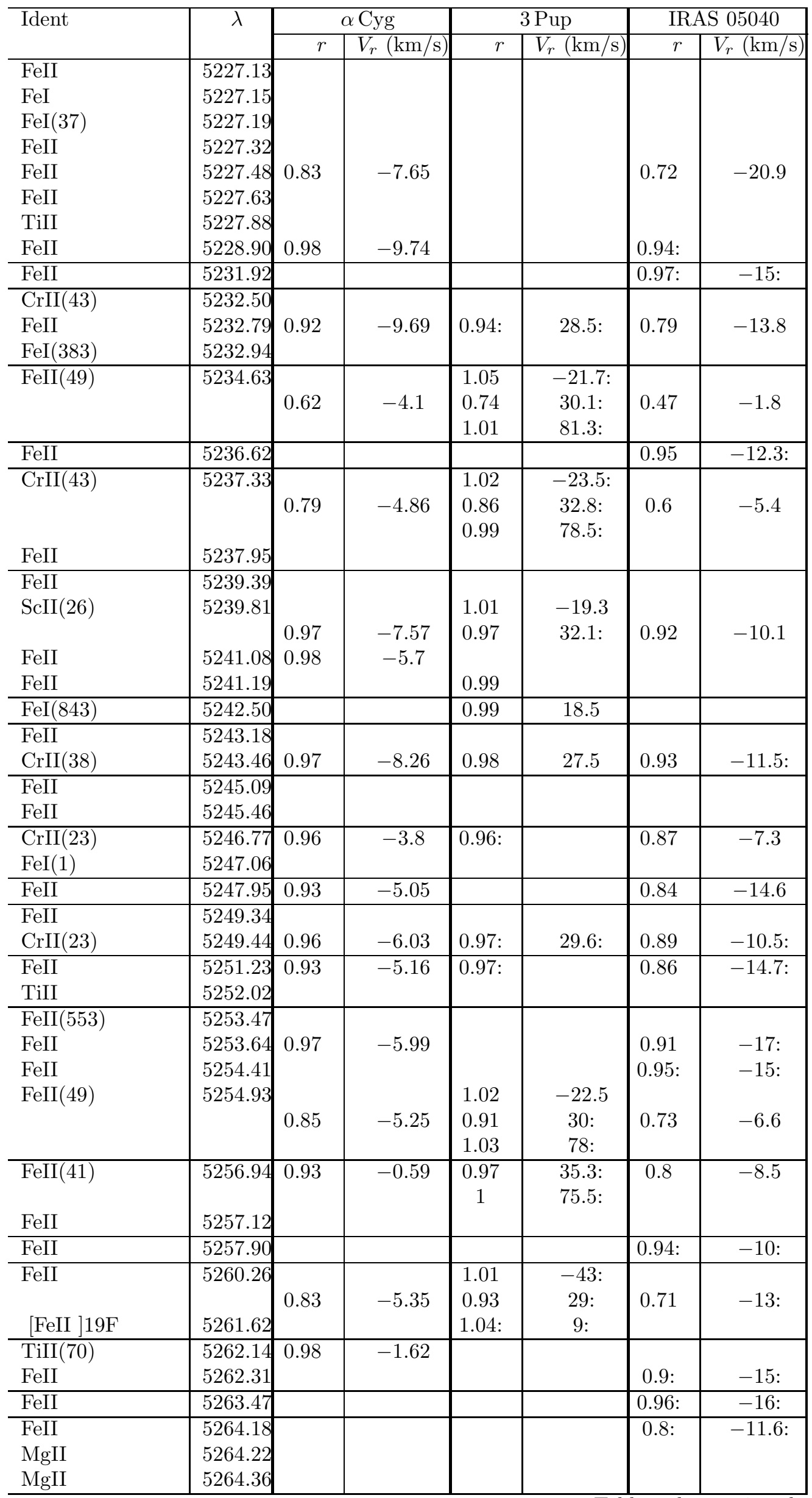




\begin{tabular}{|c|c|c|c|c|c|c|c|}
\hline \multirow[t]{2}{*}{ Ident } & \multirow[t]{2}{*}{$\lambda$} & \multicolumn{2}{|c|}{$\alpha \mathrm{Cyg}$} & \multicolumn{2}{|c|}{$3 \mathrm{Pup}$} & \multicolumn{2}{|c|}{ IRAS 05040} \\
\hline & & $r$ & $V_{r}(\mathrm{~km} / \mathrm{s})$ & $r$ & $V_{r}(\mathrm{~km} / \mathrm{s})$ & $r$ & $\overline{V_{r}(\mathrm{~km} / \mathrm{s})}$ \\
\hline $\begin{array}{l}\text { FeII(48) } \\
\text { NdIII }\end{array}$ & $\begin{array}{l}5264.81 \\
5264.96\end{array}$ & 0.82 & -6.82 & $\begin{array}{c}1 \\
0.87 \\
1.01\end{array}$ & $\begin{array}{c}-26.2: \\
24.3: \\
79.1:\end{array}$ & 0.7 & -6.6 \\
\hline $\begin{array}{l}\mathrm{FeI}(383) \\
\mathrm{CrII}\end{array}$ & $\begin{array}{l}5266.56 \\
5267.03\end{array}$ & & & 0.97: & 24: & 0.93 & $-9.7:$ \\
\hline $\begin{array}{l}\text { TiII(103) } \\
\text { FeI(15) } \\
\text { FeII } \\
\mathrm{CaI}(22) \\
\mathrm{FeI}(37)\end{array}$ & $\begin{array}{l}5268.61 \\
5269.54 \\
5270.03 \\
5270.28 \\
5270.36\end{array}$ & $\begin{array}{l}0.98 \\
0.97\end{array}$ & $\begin{array}{l}-6.63 \\
-3.7\end{array}$ & & & $\begin{array}{l}0.95: \\
0.86 \\
0.91:\end{array}$ & $\begin{array}{r}-6.3: \\
-14.6 \\
-12.8:\end{array}$ \\
\hline $\begin{array}{l}\text { FeII }(185) \\
{[\text { FeII }] 18 \mathrm{~F}}\end{array}$ & $\begin{array}{l}5272.40 \\
5273.36\end{array}$ & 0.91 & -4.75 & $\begin{array}{l}1.02 \\
0.95 \\
1.05\end{array}$ & $\begin{array}{c}-46.6: \\
22: \\
69:\end{array}$ & 0.84 & -9.9 \\
\hline $\begin{array}{l}\text { CrII(43) } \\
\text { FeII(49) }\end{array}$ & $\begin{array}{l}5274.96 \\
5276.00\end{array}$ & $\begin{array}{l}0.82 \\
0.59\end{array}$ & $\begin{array}{l}-2.36 \\
-4.43\end{array}$ & $\begin{array}{c}0.93: \\
0.73\end{array}$ & $\begin{array}{l}23.9: \\
29.2\end{array}$ & $\begin{array}{l}0.69 \\
0.46\end{array}$ & $\begin{array}{l}-3.8 \\
-1.4:\end{array}$ \\
\hline $\begin{array}{l}\text { FeII }(225) \\
\text { FeII }(184) \\
\text { CrII }\end{array}$ & $\begin{array}{l}5278.20 \\
5278.94 \\
5279.95\end{array}$ & $\begin{array}{l}0.98 \\
0.97 \\
0.91\end{array}$ & $\begin{array}{l}-5.31 \\
-4.09 \\
-4.5\end{array}$ & $\begin{array}{l}0.99: \\
1.01 \\
0.95 \\
1.02\end{array}$ & $\begin{array}{l}-21 \\
30 \\
84\end{array}$ & $\begin{array}{l}0.96 \\
0.93 \\
0.77\end{array}$ & $\begin{array}{l}-15.5 \\
-11.3 \\
-6.7:\end{array}$ \\
\hline $\mathrm{FeI}(3833)$ & 5281.79 & & & & & $0.96:$ & $-12.2:$ \\
\hline $\begin{array}{l}\text { FeI(553) } \\
\text { FeII }(41)\end{array}$ & $\begin{array}{l}5283.62 \\
5284.11\end{array}$ & 0.8 & -4.56 & $\begin{array}{c}1.07 \\
0.9 \\
1.04\end{array}$ & $\begin{array}{c}-21.5: \\
30.5: \\
73.5:\end{array}$ & $\begin{array}{l}0.93: \\
0.66\end{array}$ & $\begin{array}{l}-14.1: \\
-4.4\end{array}$ \\
\hline $\mathrm{CaII}(14)$ & 5285.34 & & & & & 0.97 & $-13:$ \\
\hline FeII & 5291.67 & 0.92 & -5.58 & 0.97 & $30.2:$ & 0.84 & -15.4 \\
\hline $\begin{array}{l}\text { NdIII } \\
\operatorname{MnII}(11)\end{array}$ & $\begin{array}{l}5294.11 \\
5294.22\end{array}$ & 0.99 & -3.57 & $\begin{array}{l}1.01 \\
0.99 \\
1.01\end{array}$ & $\begin{array}{c}-22.5: \\
-32.7: \\
66.8:\end{array}$ & 0.97: & $-14.4:$ \\
\hline $\begin{array}{l}\text { MnII(11) } \\
\text { FeI }\end{array}$ & $\begin{array}{l}5296.97 \\
5297.13\end{array}$ & 0.99 & -7.68 & & & 0.94 & $-11:$ \\
\hline $\begin{array}{l}\text { FeII } \\
\text { MnII(11) }\end{array}$ & $\begin{array}{l}5298.84 \\
5299.39\end{array}$ & 0.98 & -18.97 & & & $\begin{array}{l}0.93: \\
0.91:\end{array}$ & $\begin{array}{c}-15: \\
-19.5:\end{array}$ \\
\hline $\begin{array}{l}\text { FeI }(553) \\
\text { MnII(11) } \\
\text { VII }(54) \\
\text { FeII }(225)\end{array}$ & $\begin{array}{l}5302.30 \\
5302.43 \\
5303.26 \\
5303.40\end{array}$ & $\begin{array}{l}0.97 \\
0.99 \\
\end{array}$ & $\begin{array}{l}-5.96 \\
-7.36 \\
\end{array}$ & & & $\begin{array}{r}0.9 \\
0.94 \\
\end{array}$ & $\begin{array}{l}-16.7 \\
-17.7: \\
\end{array}$ \\
\hline FeII(184) & 5304.27 & & & & & $0.98:$ & $-15.7:$ \\
\hline $\begin{array}{l}\text { CrII }(24) \\
\text { FeII }\end{array}$ & $\begin{array}{l}5305.85 \\
5306.18\end{array}$ & 0.91 & -0.14 & $\begin{array}{l}1.02 \\
0.94\end{array}$ & $\begin{array}{c}-21.6: \\
34.9:\end{array}$ & 0.77 & $-3.8:$ \\
\hline $\begin{array}{l}\text { CaII(14) } \\
\text { FeI(36) }\end{array}$ & $\begin{array}{l}5307.22 \\
5307.37 \\
\end{array}$ & & & & & 0.96 & -6.7 \\
\hline $\operatorname{CrII}(43)$ & 5308.41 & 0.93 & -4.62 & $\begin{array}{l}1.01 \\
0.95\end{array}$ & $\begin{array}{c}-22.3: \\
33.1:\end{array}$ & 0.82 & -7.3 \\
\hline FeII & 5309.87 & & & & & $0.97:$ & $-9.2:$ \\
\hline $\mathrm{CrII}(43)$ & 5310.69 & 0.96 & -5.41 & 0.98: & $29.2:$ & 0.88 & -8.2 \\
\hline $\begin{array}{l}\text { FeII } \\
\mathrm{CrII}(43)\end{array}$ & $\begin{array}{l}5313.11 \\
5313.56\end{array}$ & 0.87 & -3.94 & $\begin{array}{l}1.02 \\
0.92 \\
1.01 \\
\end{array}$ & $\begin{array}{l}-22.4 \\
32.3: \\
78:\end{array}$ & $\begin{array}{l}0.96: \\
0.72\end{array}$ & $\begin{array}{l}-19.5: \\
-5.8:\end{array}$ \\
\hline $\begin{array}{l}\text { FeII }(225) \\
\text { FeII }\end{array}$ & $\begin{array}{l}5315.56 \\
5316.23\end{array}$ & & & & & $\begin{array}{l}0.95: \\
0.88:\end{array}$ & \\
\hline
\end{tabular}




\begin{tabular}{|c|c|c|c|c|c|c|c|}
\hline \multirow[t]{2}{*}{ Ident } & \multirow[t]{2}{*}{$\lambda$} & \multicolumn{2}{|c|}{$\alpha \mathrm{Cyg}$} & \multicolumn{2}{|c|}{$3 \mathrm{Pup}$} & \multicolumn{2}{|c|}{ IRAS 05040} \\
\hline & & $r$ & $V_{r}(\mathrm{~km} / \mathrm{s})$ & $r$ & $V_{r}(\mathrm{~km} / \mathrm{s})$ & $r$ & $V_{r}(\mathrm{~km} / \mathrm{s})$ \\
\hline FeII & 5316.62 & 0.54 & -2.97 & $\begin{array}{l}1.03 \\
0.65 \\
1.02\end{array}$ & $\begin{array}{c}-21.9 \\
31.5: \\
86.5:\end{array}$ & 0.41 & 1.7 \\
\hline FeII & 5316.78 & & & & & & \\
\hline FeII & 5318.06 & & & & & 0.92 & $-17.8:$ \\
\hline $\mathrm{CrII}(23)$ & 5318.38 & & & 0.98: & $31.6:$ & 0.93 & $-8.6:$ \\
\hline FeII & 5318.75 & & & & & & \\
\hline $\mathrm{SII}(380$ & 5320.73 & 0.99 & -4.34 & & & 0.95 & $-15:$ \\
\hline FeII & 5321.84 & & & & & $0.96:$ & $-19:$ \\
\hline FeII & 5322.23 & 0.99 & -10.72 & & & 0.95: & $-16:$ \\
\hline$\overline{\mathrm{FeI}}(553)$ & 5324.18 & 0.98 & -3.23 & 0.97 & 21.9 & 0.88 & $-11.7:$ \\
\hline FeII(49) & 5325.55 & & & 1.015 & -21 & 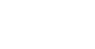 & \\
\hline & & 0.84 & -4.71 & $\begin{array}{c}0.9 \\
1.01\end{array}$ & $\begin{array}{c}31: \\
77.1\end{array}$ & 0.72 & $-5.3:$ \\
\hline $\mathrm{FeI}(15)$ & 5328.04 & 0.98 & -3.31 & & & 0.87 & $-11:$ \\
\hline FeI & 5328.53 & & & & & & \\
\hline $\mathrm{OI}(12)$ & 5329.10 & 0.97 & -1.75 & & & 0.9 & $-12.3:$ \\
\hline OI & 5329.11 & & & & & & \\
\hline OI & 5329.68 & & & & & & \\
\hline $\mathrm{OI}(12)$ & 5329.69 & & & 1.02 & -23.2 : & & \\
\hline & & 0.96 & -7.64 & 0.95 & 24.9: & 0.87 & $-14.5:$ \\
\hline OI & 5330.74 & & & $0.96:$ & 18.5: & & \\
\hline $\mathrm{OI}(12)$ & 5330.74 & 0.95 & -6.12 & 0.96 & 18.6 & 0.84 & $-13.7:$ \\
\hline$[\mathrm{FeII}] 19 \mathrm{~F}$ & 5333.65 & & & 1.03: & $27.5:$ & & \\
\hline ScII(30) & 5334.22 & & & & & 0.98 & $-12.4:$ \\
\hline $\operatorname{CrII}(43)$ & 5334.87 & & & 1.04 & -22.2 : & & \\
\hline & & 0.89 & -5.3 & 0.94 & $32.6:$ & 0.75 & -6.6 \\
\hline TiII(69) & 5336.77 & & & 1.04 & -20.6 : & & \\
\hline 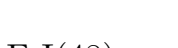 & -1 & 0.94 & -3.65 & 0.97 & $22:$ & 0.82 & -4.3 \\
\hline $\mathrm{FeI}(48)$ & 5337.73 & 0.9 & -4.38 & 0.96 & & & \\
\hline $\mathrm{CrII}(43)$ & 5337.77 & & & & & & \\
\hline FeII & 5339.59 & 0.92 & -4.41 & 0.96 & 33.3 & 0.84 & -14.6 \\
\hline $\mathrm{FeI}$ & 5339.93 & & & & & & \\
\hline FeII & 5340.30 & & & & & & \\
\hline$\overline{\mathrm{FeI}}(37)$ & 5341.03 & & & $0.99:$ & 20.1: & 0.96:- & $-10:$ \\
\hline VII(239) & 5341.22 & & & & & & \\
\hline FeII & 5344.10 & 0.99 & -4.7 & & & 0.98: & $-11:$ \\
\hline SII & 5345.72 & & & & & & \\
\hline $\operatorname{CrII}(24)$ & 5346.07 & & & & & 0.91: & \\
\hline $\mathrm{CrII}(23)$ & 5346.54 & & & & & 0.91: & \\
\hline FeII & 5346.57 & & & & & 0.92: & \\
\hline FeII & 5347.18 & & & & & & \\
\hline CrI(18) & 5348.32 & & & 0.995: & 18.9: & & \\
\hline FeII & 5353.09 & & & & & & \\
\hline FeII & 5353.22 & 0.99 & -5.13 & & & 0.96: & $-18:$ \\
\hline FeII & 5355.42 & 0.99 & -4.72 & & & $0.96:$ & $-13.5:$ \\
\hline $\operatorname{ScII}(30)$ & 5357.19 & & & & & 0.98: & $-14:$ \\
\hline FeII & 5358.87 & 0.99 & -0.13 & & & 0.96 & -11 \\
\hline FeII & 5359.24 & & & & & & \\
\hline FeII & 5360.49 & & & & & 0.98: & $-14.8:$ \\
\hline FeII & 5362.74 & & & & & & \\
\hline FeII(48) & 5362.87 & & & 1.06 & -22 : & & \\
\hline & & 0.72 & -4.07 & $\begin{array}{c}0.83 \\
1.02:\end{array}$ & $\begin{array}{l}31: \\
76:\end{array}$ & 0.58 & 0.3 \\
\hline FeII & 5362.97 & & & & & & \\
\hline
\end{tabular}




\begin{tabular}{|c|c|c|c|c|c|c|c|}
\hline \multirow[t]{2}{*}{ Ident } & \multirow[t]{2}{*}{$\lambda$} & \multicolumn{2}{|c|}{$\alpha \mathrm{Cyg}$} & \multicolumn{2}{|c|}{$3 \mathrm{Pup}$} & \multicolumn{2}{|c|}{ IRAS 05040} \\
\hline & & $r$ & $V_{r}(\mathrm{~km} / \mathrm{s})$ & $r$ & $V_{r}(\mathrm{~km} / \mathrm{s})$ & $r$ & $\overline{V_{r}(\mathrm{~km} / \mathrm{s})}$ \\
\hline $\mathrm{FeI}(1146)$ & 5364.87 & 0.99 & -7.98 & 0.995 & 19.8 & 0.96: & $-12.4:$ \\
\hline $\operatorname{FeI}(786)$ & 5365.41 & & & 0.99: & 20.8: & & \\
\hline NI & 5366.15 & & & & & & \\
\hline FeII & 5366.21 & 0.99 & -3.25 & & & 0.96 & $-15.8:$ \\
\hline $\mathrm{FeI}(1146)$ & 5367.47 & & & & & & \\
\hline VII(53) & 5367.53 & & & & & 0.93 & $-16:$ \\
\hline $\operatorname{CrII}(29)$ & 5369.35 & & & & & & \\
\hline $\mathrm{FeI}(1146)$ & 5369.96 & & & 0.98: & 20.5: & 0.9: & $-8.9:$ \\
\hline FeII & 5370.30 & 0.97 & -12.15 & & & & \\
\hline $\mathrm{FeI}(15)$ & 5371.49 & & & 1.03 & $-16:$ & & \\
\hline & & 0.98 & -6.12 & $\begin{array}{c}1: \\
1.04\end{array}$ & $\begin{array}{c}21.2: \\
71\end{array}$ & 0.91 & -10.4 \\
\hline FeI(1166) & 5373.71 & & & 0.995 & 19.5 & & \\
\hline FeII & 5375.84 & 0.98 & -3.86 & & & 0.94 & -13.6 \\
\hline $\mathrm{CI}(11)$ & 5380.32 & & & & & $0.96:$ & $-13.7:$ \\
\hline$\overline{T i I I(69)}$ & 5381.02 & 0.97 & -4.88 & $\begin{array}{l}1.02 \\
0.97 \\
1.02 \\
\end{array}$ & $\begin{array}{c}-18.7: \\
26: \\
72.8 \\
\end{array}$ & 0.89 & $-6.6:$ \\
\hline FeII(184) & 5382.40 & 0.99 & -2.61 & & & 0.97 & $-11:$ \\
\hline $\mathrm{FeI}(1146)$ & 5383.37 & 0.98 & -7.88 & 0.97: & 20.2: & 0.88 & $-12:$ \\
\hline FeII & 5387.06 & 0.92 & -5.38 & 0.97: & 35.4: & 0.86 & -14.9 \\
\hline FeII & 5388.02 & & & & & $0.98:$ & $-13.7:$ \\
\hline FeI(1062) & 5391.46 & & & 0.99: & 19.9: & & \\
\hline $\mathrm{FeI}(553)$ & 5393.17 & & & 0.99: & 19.6: & & \\
\hline FeII & 5393.59 & & & & & $0.95:$ & $-16:$ \\
\hline FeII & 5393.85 & 0.98 & -9.04 & 0.99: & & 0.94: & $-14.5:$ \\
\hline $\operatorname{CrII}(29)$ & 5395.41 & & & & & & \\
\hline FeII & 5395.86 & 0.94 & -5.39 & 0.98: & 30.2 : & 0.85 & -15.3 \\
\hline $\operatorname{TiII}(80)$ & 5396.23 & & & & & & \\
\hline TiII & 5396.56 & & & & & & \\
\hline $\mathrm{FeI}(15)$ & 5397.13 & & & 0.99: & 21.4: & 0.94 & $-9.8:$ \\
\hline FeII & 5399.56 & 1 & -9.46 & & & 0.99: & $-17:$ \\
\hline $\mathrm{FeI}(1145)$ & 5400.51 & & & 0.99 & 19.3 & $0.96:$ & $-11:$ \\
\hline MgII & 5401.52 & & & & & & \\
\hline MgII & 5401.56 & & & & & $0.87:$ & $-13.5:$ \\
\hline FeII & 5402.06 & & & 0.95: & $20.2:$ & 0.85 & $-21:$ \\
\hline FeII & 5402.87 & & & & & & \\
\hline $\mathrm{FeI}(1145)$ & 5404.12 & & & & & 0.9 & -12 \\
\hline $\operatorname{FeI}(445)$ & 5404.15 & 0.98 & -6.26 & 0.98: & 20.4: & & \\
\hline FeII & 5405.10 & 0.98 & -2.16 & & & 0.93 & $-16.5:$ \\
\hline $\mathrm{FeI}(15)$ & 5405.78 & & & 1.02: & $-12.7:$ & & \\
\hline & & 0.98 & -11.41 & $\begin{array}{c}1 \\
1.04:\end{array}$ & $\begin{array}{l}20.2: \\
68.3:\end{array}$ & 0.92 & $-14.7:$ \\
\hline $\operatorname{CrII}(23)$ & 5407.60 & 0.95 & -4.14 & 0.97: & 34.3: & 0.87 & -5.9 \\
\hline $\operatorname{FeII}(184)$ & 5408.81 & 0.97 & -2.48 & & & 0.92 & $-10:$ \\
\hline $\mathrm{CrII}(29)$ & 5409.28 & & & & & & \\
\hline $\operatorname{CrI}(18)$ & 5409.78 & & & & & 0.98 & $-10.9:$ \\
\hline FeI (1165) & 5410.91 & & & & & 0.94 & $-13.7:$ \\
\hline FeII & 5411.38 & 0.99 & -10.29 & & & 0.95 & $-15.8:$ \\
\hline $\mathrm{FeII}(48)$ & 5414.07 & 0.92 & -3.4 & $0.95:$ & & 0.81 & -6.9 \\
\hline FeII & 5414.85 & 0.97 & -1.09 & & & & \\
\hline $\mathrm{FeI}(1165)$ & 5415.20 & & & & & 0.89 & $-14.3:$ \\
\hline TiII(69) & 5418.75 & 0.98 & -4.44 & $\begin{array}{l}1.03: \\
0.98: \\
1.02:\end{array}$ & $\begin{array}{c}-18.3: \\
27.7: \\
77.7:\end{array}$ & 0.92 & -6.9 \\
\hline $\operatorname{CrII}(23)$ & 5420.92 & 0.96 & -4.94 & 0.98: & 28.4: & 0.89 & -6.7 \\
\hline
\end{tabular}




\begin{tabular}{|c|c|c|c|c|c|c|c|}
\hline \multirow[t]{2}{*}{ Ident } & \multirow[t]{2}{*}{$\lambda$} & \multicolumn{2}{|c|}{$\alpha \mathrm{Cyg}$} & \multicolumn{2}{|c|}{$3 \mathrm{Pup}$} & \multicolumn{2}{|c|}{ IRAS 05040} \\
\hline & & $r$ & $V_{r}(\mathrm{~km} / \mathrm{s})$ & $r$ & $V_{r}(\mathrm{~km} / \mathrm{s})$ & $r$ & $V_{r}(\mathrm{~km} / \mathrm{s})$ \\
\hline $\operatorname{BaII}(6)$ & 5421.05 & & & & & & \\
\hline FeI(1146) & 5424.07 & 0.98 & -2.7 & 0.98: & & 0.89 & -13.1 \\
\hline NiII & 5424.57 & & & & & & \\
\hline $\mathrm{FeII}(49)$ & 5425.26 & & & 1.02: & $-23.4:$ & & \\
\hline & & 0.86 & -4.87 & $\begin{array}{c}0.93 \\
1.02:\end{array}$ & $\begin{array}{c}29: \\
75.6:\end{array}$ & 0.75 & -6.2 \\
\hline FeII & 5427.83 & 0.96 & -4.55 & 0.98: & 24.7: & 0.91 & -15.7 \\
\hline $\operatorname{SII}(6)$ & 5428.67 & & & & & $0.95:$ & \\
\hline $\mathrm{FeI}(15)$ & 5429.70 & & & & & & \\
\hline FeII & 5429.99 & & & 1.02: & $-13.1:$ & & \\
\hline & & 0.93 & -6.41 & $\begin{array}{l}0.98: \\
1.02:\end{array}$ & $\begin{array}{c}33.9: \\
71.9\end{array}$ & 0.82 & $-17.9:$ \\
\hline SII(6) & 5432.80 & & & & & & \\
\hline $\mathrm{FeII}(55)$ & 5432.97 & & & 1.03 & $-24.7:$ & & \\
\hline & & 0.89 & -6.34 & $\begin{array}{l}0.95 \\
1.03\end{array}$ & $\begin{array}{c}27.3: \\
73.8\end{array}$ & 0.79 & -8 \\
\hline $\mathrm{FeI}(15)$ & 5434.52 & & & & & 0.97 & $-11.7:$ \\
\hline $\mathrm{OI}(11)$ & 5435.78 & & & & & $0.95:$ & \\
\hline $\mathrm{OI}(11)$ & 5436.86 & 0.98 & -5.27 & & & 0.94 & -13.5 \\
\hline FeII & 5439.71 & 0.99 & -2.08 & 0.99: & 23.7: & 0.96 & $-15.5:$ \\
\hline FeII & 5440.05 & & & & & 0.98 & \\
\hline FeII & 5442.35 & & & & & 0.95 & -11.5 \\
\hline $\mathrm{ClII}(2)$ & 5443.37 & & & & & $0.96:$ & $-11.5:$ \\
\hline FeII & 5443.45 & & & & & & \\
\hline $\mathrm{ClII}(2)$ & 5444.21 & & & & & & \\
\hline FeII & 5444.39 & 0.98 & -4.26 & & & 0.94: & $-15:$ \\
\hline $\mathrm{FeI}(1163)$ & 5445.04 & & & 0.99: & 19.7: & 0.95 & $-10.9:$ \\
\hline FeII & 5445.81 & 0.98 & -6.56 & & & 0.93 & $-16.5:$ \\
\hline $\mathrm{CrII}(35)$ & 5446.57 & & & & & & \\
\hline $\mathrm{FeI}(15)$ & 5446.92 & & & 1.02 & $-19.2:$ & & \\
\hline & & 0.99 & -3.88 & $\begin{array}{c}\text { 1: } \\
\text { 1.04: }\end{array}$ & $\begin{array}{l}\text { 24.8: } \\
\text { 68.8: }\end{array}$ & 0.94 & -10 \\
\hline FeII & 5450.10 & 0.99 & -5.49 & & & $0.97:$ & $-15:$ \\
\hline SII(6) & 5453.86 & 0.96 & -3.59 & & & 0.93 & -16.9 \\
\hline FeI & 5455.45 & & & & & & \\
\hline $\mathrm{FeI}(15)$ & 5455.61 & & & 1.03: & $-17:$ & & \\
\hline & & 0.98 & 9.5 & $\begin{array}{c}1: \\
1.02:\end{array}$ & $\begin{array}{c}25.1: \\
72:\end{array}$ & 0.93 & \\
\hline $\operatorname{CrII}(50)$ & 5455.88 & & & & & & \\
\hline FeII & 5455.93 & & & & & & \\
\hline FeII & 5457.73 & 0.98 & -6.98 & & & 0.95 & -13.4 \\
\hline FeI(1163) & 5463.28 & & & & & $0.97:$ & $-14.4:$ \\
\hline FeII & 5465.93 & 0.94 & -2.21 & & & 0.87 & -14.2 \\
\hline SiII & 5466.46 & & & & & & \\
\hline SiII & 5466.89 & & & & & & \\
\hline FeII & 5466.91 & 0.93 & -6.62 & & & 0.89 & -13.4 \\
\hline FeII & 5467.43 & & & & & & \\
\hline $\operatorname{CrII}(50)$ & 5472.60 & & & & & & \\
\hline FeII & 5472.86 & 0.99 & -11.1 & & & 0.95 & $-18:$ \\
\hline YII(27) & 5473.38 & & & & & & \\
\hline TiII(109) & 5473.52 & & & & & 0.95 & $-15:$ \\
\hline FeII & 5473.60 & & & & & & \\
\hline $\operatorname{SII}(6)$ & 5473.62 & 0.98 & -7.1 & & & & \\
\hline $\mathrm{FeI}(1062)$ & 5473.91 & & & & & 0.96: & $-14.5:$ \\
\hline FeII & 5475.83 & 0.98 & -4.58 & 0.995: & 21: & 0.95 & $-19:$ \\
\hline $\mathrm{FeI}(1062)$ & 5476.57 & & & & & & \\
\hline
\end{tabular}




\begin{tabular}{|c|c|c|c|c|c|c|c|}
\hline \multirow[t]{2}{*}{ Ident } & \multirow[t]{2}{*}{$\bar{\lambda}$} & \multicolumn{2}{|c|}{$\alpha$ Cyg } & \multicolumn{2}{|c|}{3 Pup } & \multicolumn{2}{|c|}{ IRAS 05040} \\
\hline & & $r$ & $V_{r}(\mathrm{~km} / \mathrm{s})$ & $r$ & $V_{r}(\mathrm{~km} / \mathrm{s})$ & $r$ & $\overline{V_{r}(\mathrm{~km} / \mathrm{s})}$ \\
\hline $\mathrm{NiI}(59)$ & 5476.90 & 0.99 & -5.58 & & & 0.93 & $-12.8:$ \\
\hline CrII & 5477.48 & & & & & & \\
\hline FeII(49) & 5477.65 & & & & & $0.96:$ & \\
\hline FeII & 5478.32 & & & & & & \\
\hline $\mathrm{CrII}(50)$ & 5478.37 & & & 1.01 & $-23.7:$ & & \\
\hline & & 0.92 & -5.11 & $\begin{array}{c}0.95 \\
1\end{array}$ & $\begin{array}{l}31.8: \\
80.3:\end{array}$ & 0.81 & -7.7 \\
\hline FeII & 5479.41 & 0.99 & -7.48 & & & 0.97: & \\
\hline FeII & 5480.95 & & & & & & \\
\hline FeII & 5481.29 & 0.99 & -10.18 & & & 0.94: & \\
\hline FeII & 5482.31 & 0.94 & -5.88 & 0.98: & 25.6: & 0.88 & -14.4 \\
\hline FeII & 5487.62 & 0.94 & -5.81 & $0.97:$ & 32.4: & 0.87 & -15 \\
\hline FeII & 5488.78 & 0.99 & -6.93 & & & 0.96 & $-16.3:$ \\
\hline TilI & 5490.69 & & & & & & \\
\hline FeII & 5492.08 & & & & & 0.93: & \\
\hline FeII & 5492.40 & 0.96 & -11.85 & & & 0.91: & $-15:$ \\
\hline TiII & 5492.86 & & & & & & \\
\hline FeII & 5493.83 & 0.95 & -5.42 & & & 0.89 & -17.5 \\
\hline DIB & 5494.10 & & & 0.97: & 29: & & \\
\hline VII $(53)$ & 5494.35 & & & & & & \\
\hline YII(27) & 5497.42 & & & & & & \\
\hline $\mathrm{FeI}(15)$ & 5497.52 & & & 1.01 & $-24:$ & & \\
\hline & & & & 0.98 & 30: & 0.97: & $-15.5:$ \\
\hline & & & & & & & \\
\hline FeII(204) & 5497.70 & & & & & & \\
\hline $\mathrm{CrII}(22)$ & 5497.86 & & & & & & \\
\hline FeII (24) & 5498.19 & & & & & & \\
\hline FeII & 5498.57 & 0.99 & -6.77 & & & 0.97: & \\
\hline FeII & 5501.04 & & & & & $0.96:$ & $-13.7:$ \\
\hline CrII(50) & 5502.07 & & & 1.01 & $-24.3:$ & & \\
\hline & & 0.94 & -1.79 & 0.97: & 34.3: & 0.84 & -6.2 \\
\hline FeII & 5502.68 & & & 0.96 & 25.7: & & \\
\hline FeII & 5503.21 & 0.94 & -5.11 & 0.96 & & 0.85 & $-9.3:$ \\
\hline CrII(50) & 5503.21 & & & & & & \\
\hline FeII & 5505.24 & & & & & $0.96:$ & $-15:$ \\
\hline FeII & 5506.20 & 0.88 & -4.78 & 0.95 & 28.2 & 0.78 & -14.2 \\
\hline $\mathrm{FeI}(15)$ & 5506.78 & & & 0.95 & 19.3 & & \\
\hline FeII & 5507.07 & & & & & 0.92: & $-16.6:$ \\
\hline CrII(50) & 5508.61 & 0.96 & -4.25 & 0.96 & 25.2 : & 0.85 & -6.1 \\
\hline SII(6) & 5509.72 & & & & & $0.96:$ & $-14:$ \\
\hline YII(19) & 5509.90 & 0.99 & -8.06 & & & 0.95 & -11.2 : \\
\hline FeII & 5510.67 & & & & & & \\
\hline CrII(50) & 5510.70 & 0.94 & -0.99 & 0.97 & 33.8: & 0.86 & -6.3 \\
\hline FeII & 5510.78 & & & & & & \\
\hline $\mathrm{OI}(25)$ & 5512.70 & & & & & & \\
\hline $\mathrm{CaI}(48)$ & 5512.98 & & & 0.985 & 19.1 & $0.96:$ & \\
\hline FeII(56) & 5525.13 & & & 1.01 & $-23.3:$ & & \\
\hline$\Gamma^{\prime}$ & & 0.98 & -5.56 & $0.985:$ & 25.7: & 0.91 & -9.5 \\
\hline $\operatorname{ScII}(31)$ & 5526.79 & & & 1.04 & -166 & & \\
\hline & & 0.96 & -4.61 & 0.975 & 27.7 & 0.87 & -7.9 \\
\hline & & & & 1.04 & 72.4 & & \\
\hline $\operatorname{MgI}(9)$ & 5528.41 & 0.98 & -3.25 & $0.97:$ & 27.9: & 0.87 & -14.6 \\
\hline FeII & 5529.06 & 0.98 & -8.63 & 0.97: & & & \\
\hline FeII & 5529.93 & & & & & & \\
\hline $\operatorname{TiII}(68)$ & 5529.94 & 0.97 & -4.8 & 0.98: & 26: & 0.93 & $-12:$ \\
\hline
\end{tabular}




\begin{tabular}{|c|c|c|c|c|c|c|c|}
\hline \multirow[t]{2}{*}{ Ident } & \multirow[t]{2}{*}{$\lambda$} & \multicolumn{2}{|c|}{$\alpha \mathrm{Cyg}$} & \multicolumn{2}{|c|}{$3 \mathrm{Pup}$} & \multicolumn{2}{|c|}{ IRAS 05040} \\
\hline & & $r$ & $V_{r}(\mathrm{~km} / \mathrm{s})$ & $r$ & $V_{r}(\mathrm{~km} / \mathrm{s})$ & $r$ & $V_{r}(\mathrm{~km} / \mathrm{s})$ \\
\hline FeII & 5532.09 & 0.99 & -6.6 & & & 0.95 & -16.6 \\
\hline FeII(55) & 5534.85 & 0.77 & -4.28 & $\begin{array}{l}1.06 \\
0.87 \\
1.03 \\
\end{array}$ & $\begin{array}{c}-21.4 \\
30.6: \\
74.6 \\
\end{array}$ & 0.65 & -3.1 \\
\hline $\begin{array}{l}\text { CrII }(35) \\
\text { FeII } \\
\text { FeII }(166) \\
\text { FeII }(24)\end{array}$ & $\begin{array}{l}5543.86 \\
5544.20 \\
5544.76 \\
5545.26\end{array}$ & 0.96 & -6.06 & 0.97: & 26.5: & $\begin{array}{c}0.92 \\
0.9\end{array}$ & $-16:$ \\
\hline FeI(1145) & 5546.51 & & & $0.995:$ & 20.3: & & \\
\hline $\begin{array}{l}\text { FeII } \\
\text { FeII }\end{array}$ & $\begin{array}{l}5548.21 \\
5549.00\end{array}$ & $\begin{array}{l}0.99 \\
0.98\end{array}$ & $\begin{array}{c}-5.41 \\
-5.7\end{array}$ & & & $\begin{array}{l}0.97 \\
0.93\end{array}$ & $\begin{array}{l}-12.5: \\
-14.1\end{array}$ \\
\hline $\begin{array}{l}\mathrm{FeI}(1183) \\
\mathrm{OI}(24)\end{array}$ & $\begin{array}{l}5554.90 \\
5554.95\end{array}$ & 0.98 & -4.66 & $0.985:$ & $20:$ & 0.95 & -13.2 \\
\hline $\begin{array}{l}\mathrm{MnII} \\
\mathrm{NI} \\
\mathrm{FeII}\end{array}$ & $\begin{array}{l}5559.05 \\
5560.34 \\
5561.14\end{array}$ & $\begin{array}{l}0.99 \\
0.99 \\
0.99\end{array}$ & $\begin{array}{l}-4.63 \\
-5.83 \\
-1.76\end{array}$ & & & $\begin{array}{l}0.96 \\
0.99:\end{array}$ & -3.7 \\
\hline $\begin{array}{l}\text { FeII } \\
\text { FeI }(1062) \\
\text { SII } \\
\end{array}$ & $\begin{array}{l}5563.40 \\
5563.61 \\
5564.98 \\
\end{array}$ & $\begin{array}{l}0.99 \\
0.99 \\
\end{array}$ & $\begin{array}{l}-1.78 \\
-8.75 \\
\end{array}$ & 0.995: & 20.7: & 0.98: & \\
\hline $\mathrm{FeI}(1183)$ & 5565.71 & & & 0.995: & 19.1: & & \\
\hline FeII & 5567.84 & 0.98 & -3.57 & $\begin{array}{l}1.01: \\
1: \\
1.01:\end{array}$ & $\begin{array}{c}-23.1: \\
26.2: \\
77.9:\end{array}$ & 0.96 & $-9.3:$ \\
\hline $\begin{array}{l}\operatorname{ScII}(29) \\
\mathrm{FeI}(686) \\
\mathrm{MnII} \\
\end{array}$ & $\begin{array}{l}5569.03 \\
5569.62 \\
5570.54 \\
\end{array}$ & $\begin{array}{l}0.99 \\
0.99\end{array}$ & $\begin{array}{l}-4.31 \\
-7.22\end{array}$ & 0.995: & 20.5: & $\begin{array}{c}0.98 \\
0.97:\end{array}$ & $-16.9:$ \\
\hline$\overline{F e I}(686)$ & 5572.84 & 0.99 & -4.58 & \begin{tabular}{|c|}
$1.01:$ \\
$0.99:$ \\
$1:$
\end{tabular} & $\begin{array}{c}-20.6: \\
26.4: \\
72.4:\end{array}$ & 0.94 & $-10.8:$ \\
\hline $\mathrm{FeI}(686)$ & 5576.10 & & & 0.99: & 19.3: & 0.98 & $-16:$ \\
\hline $\begin{array}{l}\text { FeII } \\
\text { MnII } \\
\text { NiI }(47) \\
\text { SII }\end{array}$ & $\begin{array}{l}5577.92 \\
5578.13 \\
5578.72 \\
5578.89\end{array}$ & 0.97 & -1.62 & 0.99 & 19.1 & & \\
\hline FeII & 5579.92 & 0.99 & -6.86 & & & 0.97: & $-10:$ \\
\hline $\begin{array}{l}\text { FeII } \\
\mathrm{CaI}(21)\end{array}$ & $\begin{array}{l}5581.63 \\
5581.97\end{array}$ & 0.99 & -3.03 & 0.99 & 18.4 & 0.98: & \\
\hline FeII & 5583.94 & 0.99 & -4.96 & & & 0.98: & $-14.2:$ \\
\hline $\begin{array}{l}\text { FeI(686) } \\
\text { FeII }\end{array}$ & $\begin{array}{l}5586.76 \\
5587.08 \\
\end{array}$ & & & $\begin{array}{c}1: \\
0.98:\end{array}$ & $\begin{array}{c}-18.7: \\
29.3:\end{array}$ & 0.89 & -9.2 \\
\hline FeII & 5588.22 & & & & & 0.93 & $-17:$ \\
\hline FeII(55) & 5591.37 & 0.99 & -4.38 & & & 0.95 & -13.2 \\
\hline AlII & 5593.30 & 0.99 & -2.47 & & & & \\
\hline $\mathrm{CaI}(21)$ & 5594.46 & 0.99 & -0.99 & & & 0.98: & \\
\hline $\begin{array}{l}\mathrm{FeI}(1183) \\
\mathrm{CaI}(21)\end{array}$ & $\begin{array}{l}5598.30 \\
5598.48\end{array}$ & & & 0.99: & 19.9: & 0.98 & $-14:$ \\
\hline $\mathrm{CaI}(21)$ & 5601.28 & & & 0.99: & 19.4: & & \\
\hline $\begin{array}{l}\mathrm{CaI}(21) \\
\mathrm{FeI}(686)\end{array}$ & $\begin{array}{l}5602.85 \\
5602.95 \\
\end{array}$ & & & & & 0.97 & $-12:$ \\
\hline $\begin{array}{l}\text { FeII(51) } \\
\text { SII(11) }\end{array}$ & $\begin{array}{l}5605.91 \\
5606.15 \\
\end{array}$ & 0.98 & -2.45 & & & 0.96 & \\
\hline $\begin{array}{l}\mathrm{FeI}(686) \\
\mathrm{NI}\end{array}$ & $\begin{array}{l}5615.64 \\
5616.55\end{array}$ & $\begin{array}{l}0.98 \\
0.98\end{array}$ & $\begin{array}{l}-5.89 \\
-3.33\end{array}$ & $\begin{array}{l}1.01 \\
0.98\end{array}$ & $\begin{array}{c}-16: \\
32:\end{array}$ & 0.9 & -11.8 \\
\hline
\end{tabular}




\begin{tabular}{|c|c|c|c|c|c|c|c|}
\hline \multirow[t]{2}{*}{ Ident } & \multirow[t]{2}{*}{$\bar{\lambda}$} & \multicolumn{2}{|c|}{$\alpha$ Cyg } & \multicolumn{2}{|r|}{3 Pup } & \multicolumn{2}{|c|}{ IRAS 05040} \\
\hline & & $r$ & $V_{r}(\mathrm{~km} / \mathrm{s})$ & $r$ & $V_{r}(\mathrm{~km} / \mathrm{s})$ & $r$ & $\overline{V_{r}(\mathrm{~km} / \mathrm{s})}$ \\
\hline SII & 5616.63 & & & & & & \\
\hline CrII(189) & 5620.63 & 0.98 & -2.48 & $\begin{array}{l}\text { 1.005: } \\
0.99:\end{array}$ & $\begin{array}{l}-20: \\
32.8:\end{array}$ & 0.95 & -13 \\
\hline $\begin{array}{l}\text { FeII } \\
\text { FeI }(686)\end{array}$ & $\begin{array}{l}5624.37 \\
5624.54\end{array}$ & 0.99 & -3.15 & $\begin{array}{c}1 \\
0.99 \\
1\end{array}$ & $\begin{array}{l}-22.8: \\
20.1: \\
71.2:\end{array}$ & 0.95: & $-14.1:$ \\
\hline FeII(57) & 5627.50 & 0.97 & -3.71 & $\begin{array}{l}1.01 \\
0.985 \\
1.005:\end{array}$ & $\begin{array}{l}-23.4 \\
31.1: \\
74.6:\end{array}$ & 0.92 & -8.6 \\
\hline$\overline{\mathrm{FeI}}(1314)$ & 5633.95 & & & 0.995: & 20.1: & 0.98: & $-15:$ \\
\hline $\begin{array}{l}\text { FeI(1087) } \\
\text { FeII } \\
\text { SII(14) } \\
\text { SII } \\
\text { CII }(15) \\
\text { ScII(29) }\end{array}$ & $\begin{array}{l}5638.27 \\
5639.69 \\
5639.97 \\
5640.33 \\
5640.55 \\
5640.98\end{array}$ & 0.97 & 1.84 & $\begin{array}{c}0.985 \\
1.01\end{array}$ & $\begin{array}{l}\text { 19.3: } \\
\text { 73.5: }\end{array}$ & $\begin{array}{l}0.94 \\
0.95: \\
0.96:\end{array}$ & $\begin{array}{l}-16: \\
-12:\end{array}$ \\
\hline VII(238) & 5642.01 & & & & & 0.98: & $-16:$ \\
\hline $\begin{array}{l}\text { FeII } \\
\text { FeII } \\
\text { FeII } \\
\text { SII(14) } \\
\text { FeII }\end{array}$ & $\begin{array}{l}5643.88 \\
5645.39 \\
5646.23 \\
5647.03 \\
5647.39\end{array}$ & $\begin{array}{l}0.97 \\
0.96 \\
0.98\end{array}$ & $\begin{array}{l}-5.41 \\
-4.32 \\
-3.81\end{array}$ & $0.985:$ & 36.8: & $\begin{array}{l}0.93 \\
0.92 \\
0.97 \\
0.97\end{array}$ & $\begin{array}{c}-13.2 \\
-14.2 \\
-16.5: \\
-13:\end{array}$ \\
\hline FeII & 5648.90 & 0.98 & -3.68 & $0.99:$ & 36.8: & 0.94 & $-15.2:$ \\
\hline FeII & 5651.54 & 0.99 & -6.85 & $0.995:$ & 37.6: & 0.98: & $-11.6:$ \\
\hline $\begin{array}{l}\text { ScII(29) } \\
\text { FeII(57) }\end{array}$ & $\begin{array}{l}5657.90 \\
5657.94\end{array}$ & 0.96 & -4.07 & $\begin{array}{l}1.03 \\
0.97 \\
1.02\end{array}$ & $\begin{array}{c}-22.8 \\
33.4 \\
73.6\end{array}$ & $\begin{array}{l}0.87 \\
0.96\end{array}$ & $\begin{array}{l}-11: \\
-18:\end{array}$ \\
\hline $\begin{array}{l}\text { SII } \\
\text { FeII } \\
\end{array}$ & $\begin{array}{l}5659.99 \\
5660.15 \\
\end{array}$ & 0.98 & -0.83 & & & 0.97: & \\
\hline $\begin{array}{l}\text { CII(15) } \\
\text { FeI(1087) } \\
\text { YII(38) }\end{array}$ & $\begin{array}{l}5662.47 \\
5662.52 \\
5662.93\end{array}$ & 0.99 & -8.51 & $\begin{array}{c}1.01 \\
0.995 \\
1.01 \\
\end{array}$ & $\begin{array}{c}-16.2 \\
23.8: \\
70.4 \\
\end{array}$ & 0.98: & $-14:$ \\
\hline SII(11) & 5664.78 & 0.99 & -6.06 & & & $0.98:$ & \\
\hline ScII $(29)$ & 5667.15 & & & $\begin{array}{l}1.02: \\
1: \\
1.02\end{array}$ & $\begin{array}{c}-19: \\
25.3: \\
76:\end{array}$ & 0.97: & -10 \\
\hline $\begin{array}{l}\text { FeII } \\
\text { ScII(29) }\end{array}$ & $\begin{array}{c}5668.64 \\
5669.03\end{array}$ & & & $\begin{array}{l}0.99 \\
1.01:\end{array}$ & $\begin{array}{c}29.6: \\
72\end{array}$ & 0.95 & $-9:$ \\
\hline SiII & 5669.56 & 0.99 & -8.08 & & & & \\
\hline FeII & 5675.26 & 0.99 & -2.47 & & & 0.96 & $-14.3:$ \\
\hline CrII(189) & 5678.39 & 0.99 & -4.84 & & & $0.94:$ & $-10.4:$ \\
\hline $\mathrm{NaI}(6)$ & 5682.63 & & & & & 0.97 & $-16.3:$ \\
\hline $\begin{array}{l}\operatorname{ScII}(29) \\
\operatorname{SiI}(11)\end{array}$ & $\begin{array}{l}5684.20 \\
5684.48\end{array}$ & & & & & $0.97:$ & $-7.9:$ \\
\hline $\mathrm{NaI}(6)$ & 5688.21 & & & & & 0.94 & -12 \\
\hline FeII & 5691.00 & & & & & $0.94:$ & $-14:$ \\
\hline AlIII & 5696.60 & 1 & -3.91 & & & & \\
\hline $\mathrm{FeI}(1183)$ & 5706.00 & & & & & 0.95 & $-7.8:$ \\
\hline
\end{tabular}




\begin{tabular}{|c|c|c|c|c|c|c|c|}
\hline \multirow[t]{2}{*}{ Ident } & \multirow[t]{2}{*}{$\lambda$} & \multicolumn{2}{|c|}{$\alpha \mathrm{Cyg}$} & \multicolumn{2}{|c|}{$3 \mathrm{Pup}$} & \multicolumn{2}{|c|}{ IRAS 05040} \\
\hline & & $r$ & $V_{r}(\mathrm{~km} / \mathrm{s})$ & $r$ & $V_{r}(\mathrm{~km} / \mathrm{s})$ & $r$ & $V_{r}(\mathrm{~km} / \mathrm{s})$ \\
\hline FeII & 5726.56 & 0.98 & -6.58 & & & 0.94: & \\
\hline FeII(57) & 5732.72 & & & & & 0.97 & $-8:$ \\
\hline FeII & 5737.90 & & & & & $0.96:$ & $-16.5:$ \\
\hline FeII & 5746.58 & 0.99 & -3.79 & & & $0.96:$ & $-13:$ \\
\hline $\operatorname{FeII}(164)$ & 5747.88 & 0.97 & -4.16 & & & 0.94 & -10 \\
\hline FeII & 5755.45 & 0.99 & -1.17 & & & 0.98 & -16.5 \\
\hline FeI(1107) & 5763.00 & & & & & 0.98: & $-10:$ \\
\hline FeII & 5776.77 & & & & & 0.97: & $-15:$ \\
\hline FeII & 5780.13 & 0.93 & -2.9 & & & $0.8:$ & $-11.9:$ \\
\hline DIB & 5780.63 & & & 0.93 & 15.9 & & \\
\hline FeII & 5783.63 & 0.97 & -4.98 & & & 0.9 & $-16.6:$ \\
\hline FeII & 5783.84 & & & & & & \\
\hline FeII & 5784.45 & 0.98 & -6.73 & & & 0.94 & $-13:$ \\
\hline FeII & 5790.69 & & & & & 0.97: & $-16.7:$ \\
\hline DIB & 5796.96 & & & 0.97: & $36:$ & 0.9 & $-2.7:$ \\
\hline $\operatorname{SiII}(8)$ & 5806.74 & & & & & 0.98: & $-11.5:$ \\
\hline FeII & 5811.63 & & & & & 0.97 & -13.5 \\
\hline $\mathrm{FeII}(24)$ & 5811.93 & & & & & & \\
\hline$\overline{F e I I}(183)$ & 5813.68 & 0.96 & -3.88 & 0.98 & 25.2 & 0.93 & $-11.4:$ \\
\hline FeII & 5813.83 & & & & & & \\
\hline $\operatorname{FeII}(164)$ & 5823.16 & 0.98 & -3.83 & 0.99 & 25.7 & 0.96 & -13.2 \\
\hline FeII & 5830.35 & & & & & $0.96:$ & $-17:$ \\
\hline$\overline{\mathrm{FeII}}(182)$ & 5835.49 & 0.98 & -1.6 & 0.99 & 25.9 & $0.96:$ & -12.6 \\
\hline FeII & 5835.61 & & & & & & \\
\hline FeII & 5838.98 & & & & & 0.98: & $-9.5:$ \\
\hline $\operatorname{CrII}(198)$ & 5840.86 & & & & & 0.98: & $-15.8:$ \\
\hline FeII & 5842.30 & 0.99 & -6.81 & & & 0.96: & $-18.5:$ \\
\hline$\overline{\mathrm{DIB}}$ & 5849.80 & & & 0.99 & 30 & 0.97 & $-2:$ \\
\hline $\mathrm{NeI}(6)$ & 5852.49 & 1 & -5.74 & & & 0.98: & $-16.5:$ \\
\hline$\overline{\mathrm{BaII}}(2)$ & 5853.68 & & & & & & \\
\hline FeII & 5854.19 & & & & & 0.96 & -16 \\
\hline FeII & 5854.19 & 0.99 & -5.44 & & & & \\
\hline $\mathrm{FeI}(1181)$ & 5859.59 & & & & & 0.98: & $-14:$ \\
\hline FeII & 5871.77 & & & & & 0.97: & $-18:$ \\
\hline HeI & 5875.61 & & & & & & \\
\hline $\mathrm{HeI}$ & 5875.62 & 0.87 & -1 & & & 0.9 & $-11.5:$ \\
\hline $\mathrm{HeI}$ & 5875.63 & & & & & & \\
\hline $\mathrm{HeI}$ & 5875.64 & & & & & & \\
\hline $\mathrm{HeI}$ & 5875.97 & & & & & & \\
\hline FeII & 5885.02 & 0.98 & -5.56 & & & 0.94: & $-14.2:$ \\
\hline $\mathrm{NaI}(1)$ & $\begin{array}{c}5889.95 \\
0.1\end{array}$ & -9.32 & 0.08 & $\begin{array}{c}1.09 \\
33: \\
1.04 \\
\end{array}$ & $\begin{array}{c}-18.6: \\
0.01 \\
73:\end{array}$ & -42 & \\
\hline FeII & 5891.33 & & & & & $0.96:$ & $-13.8:$ \\
\hline $\mathrm{NaI}(1)$ & 5895.92 & & & 1.07 & $-13.8:$ & & \\
\hline & & 0.22 & -10.05 & $\begin{array}{l}0.13 \\
1.04\end{array}$ & $\begin{array}{l}33.5: \\
76.8:\end{array}$ & 0.02 & -42 \\
\hline FeII & 5902.83 & & & & & 0.94: & $-14.1:$ \\
\hline FeII & 5944.38 & & & & & 0.97: & $-16:$ \\
\hline FeII & 5948.42 & & & & & 0.97 & $-13.2:$ \\
\hline FeII(182) & 5952.51 & 0.97 & -3.72 & & & 0.92 & -9.6 \\
\hline FeII & 5955.70 & 0.96 & -2.64 & & & 0.9 & -17.3 \\
\hline $\operatorname{SiII}(4)$ & 5957.56 & 0.87 & -4.96 & & & 0.79 & -14.3 \\
\hline FeII & 5961.71 & 0.92 & -5.25 & & & 0.85 & -13.9 \\
\hline FeII & 5965.62 & 0.98 & -7.03 & & & 0.95 & $-13.6:$ \\
\hline
\end{tabular}




\begin{tabular}{|c|c|c|c|c|c|c|c|}
\hline \multirow[t]{2}{*}{ Ident } & \multirow[t]{2}{*}{$\lambda$} & \multicolumn{2}{|c|}{$\alpha \mathrm{Cyg}$} & \multicolumn{2}{|c|}{$3 \mathrm{Pup}$} & \multicolumn{2}{|c|}{ IRAS 05040} \\
\hline & & $r$ & $V_{r}(\mathrm{~km} / \mathrm{s})$ & $r$ & $V_{r}(\mathrm{~km} / \mathrm{s})$ & $r$ & $\overline{V_{r}(\mathrm{~km} / \mathrm{s})}$ \\
\hline FeI(959) & 5976.78 & & & & & $0.98:$ & $-15:$ \\
\hline $\operatorname{SiII}(4)$ & 5978.93 & 0.82 & -4.63 & 0.92 & 34: & 0.74 & -13.7 \\
\hline $\mathrm{FeI}(1175)$ & 5983.68 & & & & & & \\
\hline FeII & 5983.86 & & & & & $0.98:$ & $-15:$ \\
\hline FeI(1260) & 5984.82 & & & & & $0.98:$ & $-13:$ \\
\hline FeII & 5988.01 & & & & & 0.97 & $-17.5:$ \\
\hline FeII(46) & 5991.38 & 0.93 & -4.23 & $\begin{array}{l}1.05: \\
0.98 \\
1.07:\end{array}$ & $\begin{array}{c}-20.3: \\
25: \\
71.2\end{array}$ & $\begin{array}{l}1.03 \\
0.88\end{array}$ & $\begin{array}{l}-34 \\
-5.5\end{array}$ \\
\hline NI & 6008.47 & 0.98 & -3.78 & & & & \\
\hline $\begin{array}{l}\text { FeI(1178) } \\
\text { PII(5) }\end{array}$ & $\begin{array}{l}6024.07 \\
6024.18\end{array}$ & 0.98 & -9.78 & & & 0.95 & -15.8 \\
\hline $\mathrm{PII}(5)$ & 6043.12 & 0.99 & -5.7 & & & $0.98:$ & $-17.6:$ \\
\hline $\begin{array}{l}\mathrm{FeII}(200) \\
\mathrm{OI}(22)\end{array}$ & $\begin{array}{l}6045.47 \\
6046.44\end{array}$ & 0.98 & -1.01 & & & 0.96 & \\
\hline FeII & 6049.45 & 0.99 & -7.64 & & & $0.98:$ & \\
\hline SI(10) & 6052.66 & & & & & 0.98 & $-11:$ \\
\hline CrII(105) & 6053.47 & 0.98 & -4.88 & 0.99 & $25.7:$ & 0.94 & -7.3 \\
\hline FeII & 6060.98 & 0.98 & -4.85 & & & $0.96:$ & $-13.6:$ \\
\hline$\overline{\mathrm{FeI}}(207)$ & 6065.49 & & & $\begin{array}{c}1.01: \\
1: \\
1.01 \\
\end{array}$ & $\begin{array}{c}-13.2: \\
27: \\
63.9:\end{array}$ & 0.99: & $-13:$ \\
\hline $\begin{array}{l}\text { FeII } \\
\text { CrII(197) } \\
\text { CrII(105) }\end{array}$ & $\begin{array}{l}6069.67 \\
6069.69 \\
6070.08\end{array}$ & 0.99 & -1 & & & 0.98: & $-15:$ \\
\hline FeII & 6071.42 & 0.99 & -4.62 & & & $0.98:$ & $-15:$ \\
\hline $\mathrm{NeI}$ & 6074.37 & 0.99 & -3.86 & & & & \\
\hline FeI(1259) & 6078.50 & & & & & $0.98:$ & $-14:$ \\
\hline FeII & 6082.89 & & & & & $0.98:$ & $-14.5:$ \\
\hline FeII(46) & 6084.11 & 0.95 & -4.79 & $\begin{array}{l}1.04 \\
0.98 \\
1.03\end{array}$ & $\begin{array}{l}-21.5 \\
26.9 \\
72.5\end{array}$ & $\begin{array}{l}1.04 \\
0.88\end{array}$ & $\begin{array}{c}-34.2 \\
-8.1\end{array}$ \\
\hline $\operatorname{SII}(26)$ & 6102.28 & & & & & 0.97 & $-14:$ \\
\hline FeII(200) & 6103.50 & 0.97 & -4.54 & & & 0.94 & $-10.8:$ \\
\hline NI1(45) & 6108.12 & & & $\begin{array}{c}\text { 1.01: } \\
\text { 1: } \\
1.01: \\
\end{array}$ & $\begin{array}{l}-5: \\
25: \\
59:\end{array}$ & & \\
\hline FeII(46) & 6113.32 & 0.97 & -4.34 & $\begin{array}{l}1.01 \\
0.98 \\
1.01\end{array}$ & $\begin{array}{c}-22.1 \\
25.7: \\
74.4\end{array}$ & 0.92 & -6.5 \\
\hline FeII & 6116.06 & 0.99 & -4.08 & $\begin{array}{c}1 \\
0.99: \\
1 \\
\end{array}$ & $\begin{array}{c}-18.4: \\
23: \\
76.7:\end{array}$ & 0.97: & $-13.3:$ \\
\hline $\mathrm{CaI}(3)$ & 6122.22 & & & $\begin{array}{c}1.02: \\
1: \\
1.015:\end{array}$ & $\begin{array}{c}-21: \\
19.1: \\
72:\end{array}$ & & \\
\hline $\operatorname{MnII}(13)$ & 6122.45 & 0.99 & -5.11 & & & $0.96:$ & $-18.5:$ \\
\hline $\operatorname{MnII}(13)$ & 6122.80 & & & & & & \\
\hline $\operatorname{MnII}(13)$ & 6123.16 & & & & & & \\
\hline NiII & 6124.89 & 0.99 & -4.13 & & & $0.96:$ & $-13.6:$ \\
\hline $\begin{array}{l}\text { MnII(13) } \\
\operatorname{MnII}(13)\end{array}$ & $\begin{array}{l}6125.86 \\
6126.21\end{array}$ & & & & & $0.87:$ & $-9:$ \\
\hline FeI(1017) & 6127.91 & & & $\begin{array}{c}\text { 1.005: } \\
1: \\
1.01:\end{array}$ & $\begin{array}{c}-20: \\
19.4: \\
54:\end{array}$ & & \\
\hline
\end{tabular}




\begin{tabular}{|c|c|c|c|c|c|c|c|}
\hline \multirow[t]{2}{*}{ Ident } & \multirow[t]{2}{*}{$\lambda$} & \multicolumn{2}{|c|}{$\alpha \mathrm{Cyg}$} & \multicolumn{2}{|c|}{$3 \mathrm{Pup}$} & \multicolumn{2}{|c|}{ IRAS 05040} \\
\hline & & $r$ & $V_{r}(\mathrm{~km} / \mathrm{s})$ & $r$ & $V_{r}(\mathrm{~km} / \mathrm{s})$ & $r$ & $V_{r}(\mathrm{~km} / \mathrm{s})$ \\
\hline $\operatorname{MnII}(13)$ & 6128.73 & & & & & & \\
\hline MnII(13) & 6129.02 & & & & & & \\
\hline CrII(105) & 6129.23 & & & 1.01: & $-8.7:$ & & \\
\hline & & 0.99 & 1.81 & $\begin{array}{c}1: \\
1.01:\end{array}$ & $\begin{array}{l}\text { 41.9: } \\
\text { 88.1: }\end{array}$ & $0.98:$ & \\
\hline MnII(13) & 6129.26 & & & & & & \\
\hline FeII $(46)$ & 6129.70 & & & & & 0.97: & \\
\hline FeI(169) & 6137.62 & & & 1: & 24.3: & 0.98: & \\
\hline $\mathrm{FeI}(207)$ & 6137.69 & & & & & & \\
\hline$\overline{\mathrm{BaII}(2)}$ & 6141.71 & 0.99 & -9.05 & $\begin{array}{l}1.02: \\
0.99: \\
1.02:\end{array}$ & $\begin{array}{c}-19.7: \\
29.3: \\
69.3:\end{array}$ & $0.96:$ & $-9.7:$ \\
\hline $\operatorname{SiI}(30)$ & 6142.49 & & & & & & \\
\hline $\operatorname{NeI}(1)$ & 6143.06 & 0.99 & -3.73 & & & 0.97 & $-21:$ \\
\hline CrII(105) & 6147.15 & & & & & 0.97: & $-17:$ \\
\hline FeII(74) & 6147.74 & & & 1.04 & -20.6 & 1.01 & -32.6 \\
\hline & & 0.86 & -4.35 & 0.92 & 25.4 & 0.76 & -3.6 \\
\hline & & & & 1.03 & 66.4 & & \\
\hline $\mathrm{FeII}(74)$ & 6149.26 & 0.86 & -4.85 & 0.93 & 28.2 & 1.03 & -36.3 \\
\hline & & & & 1.02 & 74.2 & 0.75 & -4.4 \\
\hline SiI & 6155.13 & & & & & & \\
\hline OI & 6155.96 & & & & & & \\
\hline OI & 6155.97 & 0.94 & -1.71 & & & 0.81 & -11.7 \\
\hline OI & 6155.99 & & & & & & \\
\hline OI & 6156.74 & & & & & & \\
\hline OI & 6156.76 & & & & & & \\
\hline OI & 6156.78 & 0.91 & -7.62 & & & 0.76 & -13 \\
\hline OI & 6158.17 & & & & & & \\
\hline OI & 6158.19 & 0.89 & -6.5 & & & 0.74 & -10 \\
\hline $\mathrm{NaI}$ & 6160.75 & 0.99 & -5.76 & & & 0.98: & \\
\hline $\mathrm{CaI}(3)$ & 6162.18 & & & $\begin{array}{l}\text { 1.01: } \\
0.98 \\
1.01:\end{array}$ & $\begin{array}{c}-14: \\
23: \\
67.6:\end{array}$ & 0.96: & $-8.5:$ \\
\hline $\mathrm{NeI}$ & 6163.59 & & & & & & \\
\hline FeII & 6163.60 & 0.99 & -3.08 & & & & \\
\hline $\mathrm{CrII}(187)$ & 6179.10 & & & & & & \\
\hline FeII(163) & 6179.38 & 0.97 & -7.29 & & & 0.92 & -10.4 \\
\hline FeI(959) & 6187.99 & & & 0.99: & 18.4: & & \\
\hline FeI(169) & 6191.57 & & & $\begin{array}{l}1.03 \\
1.01: \\
1.02:\end{array}$ & $\begin{array}{c}-15.4: \\
18.5:\end{array}$ & & \\
\hline CrII(105) & 6195.18 & & & & & 0.97: & $-15:$ \\
\hline DIB & 6195.96 & & & & & 0.93 & -1.3 \\
\hline$\overline{\mathrm{DIB}}$ & 6198.83 & & & & & & \\
\hline FeII(162) & 6199.18 & 0.98 & -6.08 & 0.99: & 26.4: & 0.96 & -13.5 \\
\hline$\overline{\mathrm{DIB}}$ & 6203.08 & & & 0.98 & 30.3: & 0.94 & $-1.6:$ \\
\hline $\mathrm{FeI}(62)$ & 6213.44 & & & 0.99: & 18.5: & & \\
\hline $\mathrm{NeI}$ & 6217.28 & 1 & -4.2 & & & & \\
\hline$\overline{\mathrm{FeI}}(62)$ & 6219.29 & & & $\begin{array}{c}\text { 1.01: } \\
\text { 1.005: } \\
1.01:\end{array}$ & $\begin{array}{l}-9: \\
33: \\
70:\end{array}$ & & \\
\hline FeII & 6224.64 & & & & & 0.98: & $-14.5:$ \\
\hline $\mathrm{CrII}(105)$ & 6226.66 & & & & & 0.98: & $-11:$ \\
\hline FeII $(34)$ & 6229.35 & & & & & 0.98 & $-8.9:$ \\
\hline$\overline{\mathrm{FeI}}(207)$ & 6230.73 & & & $\begin{array}{c}1.02: \\
1:\end{array}$ & $\begin{array}{c}-3: \\
20.9:\end{array}$ & 0.98 & \\
\hline
\end{tabular}




\begin{tabular}{|c|c|c|c|c|c|c|c|}
\hline \multirow[t]{2}{*}{ Ident } & \multirow[t]{2}{*}{$\bar{\lambda}$} & \multicolumn{2}{|c|}{$\alpha$ Cyg } & \multicolumn{2}{|c|}{3 Pup } & \multicolumn{2}{|c|}{ IRAS 05040} \\
\hline & & $r$ & $V_{r}(\mathrm{~km} / \mathrm{s})$ & $r$ & $V_{r}(\mathrm{~km} / \mathrm{s})$ & $r$ & $\overline{V_{r}(\mathrm{~km} / \mathrm{s})}$ \\
\hline AlII(10) & 6231.75 & 0.98 & -2.9 & 1.01: & 62: & 0.98: & $-10:$ \\
\hline$\overline{\mathrm{FeI}(816)}$ & 6232.65 & & & $0.99:$ & 18: & & \\
\hline $\begin{array}{l}\text { FeII } \\
\text { FeII }\end{array}$ & $\begin{array}{l}6233.53 \\
6233.76\end{array}$ & 0.98 & -3 & & & 0.94 & $-11.8:$ \\
\hline $\begin{array}{l}\operatorname{SiI}(28) \\
\end{array}$ & 6237.32 & & & & & $0.98:$ & $-15:$ \\
\hline FeII(74) & 6238.39 & 0.85 & -4.26 & $\begin{array}{l}1.04 \\
0.93 \\
1.02\end{array}$ & $\begin{array}{c}-19.1 \\
25.9: \\
71.9\end{array}$ & $\begin{array}{l}1.07 \\
0.73\end{array}$ & $\begin{array}{l}-35.6 \\
-3.1\end{array}$ \\
\hline $\begin{array}{l}\text { CrII(105) } \\
\text { FeII( } 74) \\
\end{array}$ & $\begin{array}{l}6239.77 \\
6239.95 \\
\end{array}$ & 0.94 & -13.8 & 0.98: & 25: & 0.88 & -10.7 \\
\hline AlII(10) & 6243.37 & 0.97 & -5.52 & & & 0.97 & $-18:$ \\
\hline SiI(28) & 6243.81 & & & & & $0.98:$ & $-16:$ \\
\hline$\overline{\mathrm{ScII}(28)}$ & 6245.64 & & & 1: & & 0.96 & $-9.4:$ \\
\hline $\operatorname{FeII}(74)$ & 6247.56 & 0.77 & -4.69 & $\begin{array}{l}1.07 \\
0.87\end{array}$ & $\begin{array}{c}-21.3 \\
27.7\end{array}$ & $\begin{array}{l}1.06 \\
0.65\end{array}$ & $\begin{array}{c}-33.7 \\
-1.8\end{array}$ \\
\hline $\mathrm{NeI}(5)$ & 6266.50 & 0.99 & -3.75 & & & $0.98:$ & $-15.5:$ \\
\hline $\begin{array}{l}\text { DIB } \\
\text { FeII } \\
\text { FeI }\end{array}$ & $\begin{array}{l}6269.75 \\
6269.97 \\
6270.23\end{array}$ & 0.99 & -2.7 & & & 0.93 & -7.8 \\
\hline$\overline{\mathrm{DiB}}$ & 6283.85 & & & 0.93: & $35:$ & $0.83:$ & $-3:$ \\
\hline FeII & 6291.83 & 0.98 & -4.55 & & & 0.92 & $-16:$ \\
\hline$[\mathrm{OI}] 1 \mathrm{~F}$ & 6300.30 & & & \begin{tabular}{|l|}
1.11 \\
1.09 \\
1.13 \\
\end{tabular} & $\begin{array}{c}3 \\
30: \\
55 \\
\end{array}$ & & \\
\hline $\begin{array}{l}\text { FeII(200) } \\
\text { SII(19) }\end{array}$ & $\begin{array}{l}6305.30 \\
6305.48\end{array}$ & 0.95 & -2.62 & & & 0.91 & -6.1 \\
\hline $\begin{array}{l}\text { SII }(26) \\
\end{array}$ & 6312.66 & 0.99 & -7.57 & & & $0.98:$ & $-6.4:$ \\
\hline $\begin{array}{l}\text { FeII } \\
\text { FeII }\end{array}$ & $\begin{array}{l}6317.39 \\
6317.98\end{array}$ & 0.91 & -6.48 & & & 0.8 & $-9.7:$ \\
\hline $\begin{array}{l}\mathrm{MgII} \\
\mathrm{MgII} \\
\mathrm{SiII}(2)\end{array}$ & $\begin{array}{l}6346.74 \\
6346.96 \\
6347.11\end{array}$ & 0.47 & -5.11 & 0.87 & 33.5: & 0.39 & -7.5 \\
\hline $\begin{array}{l}\text { FeII } \\
\text { FeI(13) }\end{array}$ & $\begin{array}{l}6357.16 \\
6358.70\end{array}$ & 0.98 & -6.81 & $\begin{array}{l}0.995: \\
1.015: \\
1.01: \\
1.02:\end{array}$ & $\begin{array}{c}27.5: \\
-6: \\
27: \\
61:\end{array}$ & $\begin{array}{l}0.95 \\
0.98:\end{array}$ & $\begin{array}{l}-14.1: \\
-8.5:\end{array}$ \\
\hline$[\mathrm{OI}] 1 \mathrm{~F}$ & 6363.78 & & & $\begin{array}{l}1.04: \\
\text { 1.04: } \\
\text { 1.04: }\end{array}$ & $\begin{array}{l}\text { 1: } \\
\text { 28: } \\
55:\end{array}$ & & \\
\hline $\begin{array}{l}\text { DIB } \\
\text { FeII }\end{array}$ & $\begin{array}{l}6367.25 \\
6367.41\end{array}$ & 0.99 & -6.7 & & & $0.96:$ & $-0.2:$ \\
\hline $\begin{array}{l}\text { FeII } \\
\text { FeII(40) }\end{array}$ & $\begin{array}{l}6369.06 \\
6369.46\end{array}$ & 0.96 & -4.77 & $\begin{array}{l}\text { 1.045: } \\
\text { 1: } \\
1.04:\end{array}$ & $\begin{array}{c}-19.6: \\
26: \\
65:\end{array}$ & 0.91 & $-4.9:$ \\
\hline $\begin{array}{l}\text { SiII(2) } \\
\text { FeII }\end{array}$ & $\begin{array}{l}6371.37 \\
6371.72 \\
\end{array}$ & 0.53 & -4.14 & 0.74 & 34.9: & 0.46 & -6.4 \\
\hline $\begin{array}{l}\text { FeII } \\
\text { DIB }\end{array}$ & $\begin{array}{l}6375.79 \\
6375.95\end{array}$ & 0.99 & $\begin{array}{l}-7.23 \\
\end{array}$ & 0.99: & 31: & $0.96:$ & \\
\hline DIB & 6379.29 & 0.99 & -7.44 & $0.98:$ & 25: & 0.94 & 0: \\
\hline $\mathrm{NeI}(3)$ & 6382.99 & & & & & 0.97: & $-10:$ \\
\hline $\begin{array}{l}\text { FeII } \\
\text { SII }\end{array}$ & $\begin{array}{l}6383.72 \\
6384.89\end{array}$ & 0.95 & -5.39 & 0.98 & 27.1: & 0.88 & \\
\hline FeII & 6385.45 & 0.97 & -4.48 & & & 0.92 & -12.6 \\
\hline
\end{tabular}




\begin{tabular}{|c|c|c|c|c|c|c|c|}
\hline \multirow[t]{2}{*}{ Ident } & \multirow[t]{2}{*}{$\lambda$} & \multicolumn{2}{|c|}{$\alpha \mathrm{Cyg}$} & \multicolumn{2}{|c|}{$3 \mathrm{Pup}$} & \multicolumn{2}{|c|}{ IRAS 05040} \\
\hline & & $r$ & $V_{r}(\mathrm{~km} / \mathrm{s})$ & $r$ & $V_{r}(\mathrm{~km} / \mathrm{s})$ & $r$ & $\overline{V_{r}(\mathrm{~km} / \mathrm{s})}$ \\
\hline FeII $(203)$ & 6386.71 & 0.99 & -10.66 & & & 0.98: & $-16:$ \\
\hline $\mathrm{FeI}(168)$ & 6393.61 & & & $\begin{array}{c}1.01 \\
1 \\
1.01\end{array}$ & $\begin{array}{c}-13: \\
27: \\
65:\end{array}$ & 0.99: & $-12:$ \\
\hline $\mathrm{FeI}(816)$ & 6400.00 & & & $\begin{array}{c}1.02 \\
1 \\
1.03:\end{array}$ & $\begin{array}{c}5.2 \\
33 \\
69.7\end{array}$ & 0.97 & $-13.5:$ \\
\hline $\mathrm{NeI}(1)$ & 6402.25 & 0.98 & -5.14 & & & 0.94 & -18.1 \\
\hline $\begin{array}{l}\text { FeII }(74) \\
\text { FeI }(816)\end{array}$ & $\begin{array}{l}6407.25 \\
6408.03\end{array}$ & 0.98 & -2.7 & $\begin{array}{l}1.01: \\
0.99: \\
1.005 \\
0.98:\end{array}$ & $\begin{array}{c}-21.7 \\
39.5 \\
73.9 \\
20.1:\end{array}$ & 0.95: & $-10:$ \\
\hline $\mathrm{FeI}(816)$ & 6411.65 & & & $\begin{array}{c}1.005: \\
0.98 \\
1\end{array}$ & $\begin{array}{c}-23: \\
17: \\
59:\end{array}$ & & \\
\hline SII(19) & 6413.71 & & & & & 0.98: & $-14:$ \\
\hline FeII & 6416.92 & & & & & $0.8:$ & $-5:$ \\
\hline $\begin{array}{l}\mathrm{FeI}(62) \\
\mathrm{FeII}(40)\end{array}$ & $\begin{array}{l}6430.85 \\
6432.68 \\
\\
6433.81\end{array}$ & $\begin{array}{l}0.92 \\
0.99\end{array}$ & $\begin{array}{l}-2.49 \\
-9.17\end{array}$ & $\begin{array}{l}\text { 0.99: } \\
\text { 1.13: } \\
0.98: \\
1.12:\end{array}$ & $\begin{array}{c}18.3: \\
-19.6 \\
26 \\
67.1\end{array}$ & $\begin{array}{l}0.86 \\
0.97\end{array}$ & $\begin{array}{c}0: \\
-9.6:\end{array}$ \\
\hline $\mathrm{CaI}(18)$ & 6439.08 & & & 0.98: & 17.6: & & \\
\hline FeII & 6442.96 & 0.98 & -4.09 & & & 0.93 & -10.2 : \\
\hline FeII $(199)$ & 6446.41 & 0.96 & -4.5 & 0.98: & 31.2: & 0.93 & $-5.8:$ \\
\hline $\begin{array}{l}\text { OI } \\
\text { OI } \\
\text { FeII } \\
\text { OI } \\
\text { FeII }(74)\end{array}$ & $\begin{array}{l}6453.60 \\
6454.44 \\
6455.84 \\
6455.98 \\
6456.38 \\
6456.88\end{array}$ & 0.73 & -4.79 & $\begin{array}{l}1.05 \\
0.83 \\
1.04\end{array}$ & $\begin{array}{c}-19.7: \\
\text { 28.1: } \\
74.2:\end{array}$ & $\begin{array}{c}0.92 \\
0.9 \\
0.9 \\
0.58\end{array}$ & $\begin{array}{c}-14.6: \\
-11.3 \\
-21.8: \\
0.6\end{array}$ \\
\hline $\mathrm{CaI}(18)$ & 6462.56 & & & \begin{tabular}{|l|}
0.98 \\
\end{tabular} & 20.4: & 0.97: & $-12:$ \\
\hline $\mathrm{CaI}(18)$ & 6471.66 & & & 0.99 & 18.9: & & \\
\hline $\begin{array}{l}\text { NI } \\
\text { FeII } \\
\text { NI } \\
\text { NI } \\
\text { NI }\end{array}$ & $\begin{array}{l}6481.71 \\
6482.20 \\
6482.70 \\
6483.75 \\
6484.81\end{array}$ & 0.91 & -0.45 & 0.96: & & 0.85 & -2.4 \\
\hline FeII & 6493.03 & & & & & 0.92: & $-12:$ \\
\hline FeII & 6531.16 & & & & & 0.98: & $-13.5:$ \\
\hline FeII & 6541.36 & 0.99 & -3.01 & & & 0.97: & -11.7 \\
\hline $\begin{array}{l}\text { MgII } \\
\text { MgII }\end{array}$ & $\begin{array}{l}6545.94 \\
6545.99\end{array}$ & 0.88 & -6.97 & & & 0.82 & $-13.9:$ \\
\hline $\begin{array}{l}\text { TiII } \\
\text { FeII } \\
\text { HI }\end{array}$ & $\begin{array}{l}6559.59 \\
6562.20 \\
6562.80\end{array}$ & 0.73 & -29.89 & $\begin{array}{l}1.92 \\
0.85 \\
2.75:\end{array}$ & $\begin{array}{l}-34 \\
29: \\
84:\end{array}$ & $\begin{array}{l}1.92 \\
0.72 \\
1.53\end{array}$ & $\begin{array}{c}-49.9 \\
-20.6 \\
12.6\end{array}$ \\
\hline FeII & 6571.42 & 0.99 & -6.33 & & & $0.96:$ & \\
\hline FeII & 6574.47 & & & & & 0.98: & $-16:$ \\
\hline $\mathrm{CII}(2)$ & 6578.05 & 0.98 & -3.65 & & & 0.96: & $-16.8:$ \\
\hline $\mathrm{CII}(2)$ & 6582.88 & 0.98 & -9.13 & & & 0.97: & $-14.5:$ \\
\hline
\end{tabular}




\begin{tabular}{|c|c|c|c|c|c|c|c|}
\hline \multirow[t]{2}{*}{ Ident } & \multirow[t]{2}{*}{$\lambda$} & \multicolumn{2}{|c|}{$\alpha \mathrm{Cyg}$} & \multicolumn{2}{|c|}{$3 \mathrm{Pup}$} & \multicolumn{2}{|c|}{ IRAS 05040} \\
\hline & & $r$ & $V_{r}(\mathrm{~km} / \mathrm{s})$ & $r$ & $V_{r}(\mathrm{~km} / \mathrm{s})$ & $r$ & $\overline{V_{r}(\mathrm{~km} / \mathrm{s})}$ \\
\hline FeII & 6586.70 & & & & & 0.94: & $-15:$ \\
\hline $\mathrm{CI}(22)$ & 6587.61 & & & & & 0.94: & $-13:$ \\
\hline DIB & 6613.56 & 0.99 & -12.44 & 0.97 & $32:$ & 0.9 & 1: \\
\hline FeII(210) & 6627.26 & 0.98 & -4.83 & 0.98 & 29: & 0.97: & $-11:$ \\
\hline $\mathrm{NiI}(43)$ & 6643.64 & & & $0.98:$ & 18.8: & & \\
\hline NI & 6644.96 & 0.99 & -5.45 & & & & \\
\hline Eu2(8) & 6645.11 & & & & & 0.97 & $-15.7:$ \\
\hline NI & 6653.45 & 0.99 & -1.71 & & & & \\
\hline SiII & 6660.52 & 0.99 & -6.21 & & & & \\
\hline DIB & 6660.64 & & & 0.99: & 34: & 0.96 & $-7:$ \\
\hline$\overline{F e I(111) ~}$ & 6663.45 & & & 0.98: & 18.8: & & \\
\hline $\begin{array}{l}\text { SiII } \\
\text { DIB }\end{array}$ & $\begin{array}{l}6671.88 \\
6672.15\end{array}$ & 0.99 & -6.81 & & & 0.96 & $-17:$ \\
\hline FeII & 6677.31 & & & & & $0.95:$ & \\
\hline $\operatorname{FeI}(268)$ & 6677.99 & & & 0.98: & 19.4: & & \\
\hline $\mathrm{HeI}$ & 6678.15 & 0.94 & -4.77 & & & 0.95 & \\
\hline FeII & 6679.74 & & & & & 0.98: & \\
\hline TiII & 6680.13 & & & & & & \\
\hline NI & 6706.11 & 0.99 & -5.39 & & & & \\
\hline FeII & 6708.89 & 0.99 & -10.03 & & & & \\
\hline $\mathrm{CaI}(32)$ & 6717.69 & & & $\begin{array}{c}1.01 \\
0.98: \\
1.005\end{array}$ & $\begin{array}{c}-17: \\
27: \\
72:\end{array}$ & 0.98: & \\
\hline TiII & 6717.79 & & & & & & \\
\hline NI & 6722.61 & 0.98 & -4.91 & & & & \\
\hline $\mathrm{SI}(8)$ & 6748.79 & & & & & 0.97 & $-16.4:$ \\
\hline $\mathrm{FeI}(111)$ & 6750.16 & & & 0.98 & 19.6 & & \\
\hline $\mathrm{SI}(8)$ & 6757.16 & & & & & 0.96 & $-15.5:$ \\
\hline NiI(57) & 6767.78 & & & $\begin{array}{l}1.01: \\
0.98: \\
1.01:\end{array}$ & $\begin{array}{c}-5: \\
19.1: \\
59:\end{array}$ & & \\
\hline NiI(127) & 6772.32 & & & 0.99 & 19.2 & & \\
\hline FeI(1173) & 6843.66 & & & 0.97 & 19.1 & & \\
\hline $\mathrm{FeI}(1173)$ & 6858.15 & & & 0.98 & 19.3 & & \\
\hline FeII & 6862.53 & 0.99 & -5.32 & & & 0.97 & $-13.7:$ \\
\hline FeII & 6922.03 & & & & & 0.9 & -15.5 \\
\hline
\end{tabular}

